\title{
Process Management and Quality Assurance for Intracranial Stereotactic Treatment
}

\section{NEDERLANDSE COMMISSIE VOOR STRALINGSDOSIMETRIE}

Report 25 of the Netherlands Commission on Radiation Dosimetry October 2015

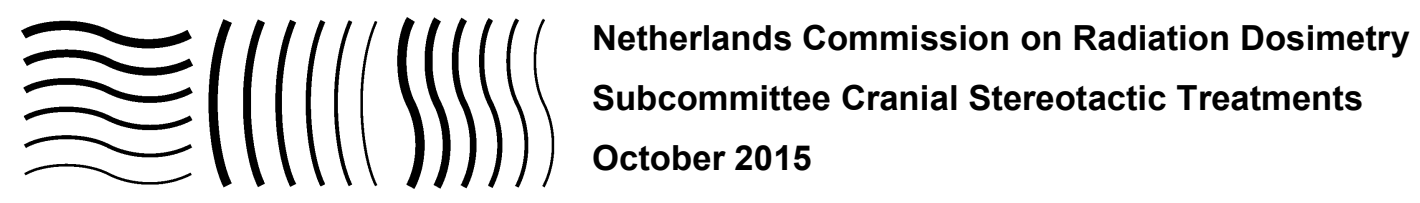




\section{Disclaimer regarding NCS Reports}

The NCS frequently publishes reports for fellow professionals in which recommendations are given for various quality control procedures or otherwise. The members of the NCS board and the members of the concerning subcommittee do not claim any authority exceeding that of their professional expertise. Responsibility on how the NCS recommendations are implemented lies with the user, taking into account the practice in his/her institution.

This report should be revised before November 2020 


\section{Preface}

The Nederlandse Commissie voor Stralingsdosimetrie (NCS, Netherlands Commission on Radiation Dosimetry, http://www.radiationdosimetry.org) was officially established on 3 September 1982 with the aim of promoting the appropriate use of dosimetry of ionising radiation both for scientific research and practical applications. The NCS is chaired by a board of scientists, installed upon the suggestion of the supporting societies, including the Nederlandse Vereniging voor Radiotherapie en Oncologie (Netherlands Society for Radiotherapy and Oncology), the Nederlandse Vereniging voor Nucleaire Geneeskunde (Dutch Society of Nuclear Medicine), the Nederlandse Vereniging voor Klinische Fysica (Dutch Society for Medical Physics), the Nederlandse Vereniging voor Radiobiologie (Netherlands Radiobiological Society), the Nederlandse Vereniging voor Stralingshygiëne (Netherlands Society for Radiological Protection), the Nederlandse Vereniging voor Medische Beeldvorming en Radiotherapie (Dutch Society for Medical Imaging and Radiotherapy), the Nederlandse Vereniging van Klinisch Fysisch Medewerkers (Dutch Society for Medical Physics Engineers), the Nederlandse Vereniging voor Radiologie (Radiological Society of the Netherlands) and the Belgische Vereniging voor Ziekenhuisfysici/Société Belge des Physiciens des Hôpitaux (Belgian Hospital Physicists Association). To pursue its aims, the NCS accomplishes the following tasks: participation in dosimetry standardisation and promotion of dosimetry intercomparisons, drafting of dosimetry protocols, collection and evaluation of physical data related to dosimetry. Furthermore, the commission shall maintain or establish links with national and international organisations concerned with ionising radiation and promulgate information on new developments in the field of radiation dosimetry.

Current members of the board of the NCS

J.B. van de Kamer, Chairman T.W.M. Grimbergen, Vice-Chairman J. de Pooter, Secretary J.M.J. Hermans, Treasurer

A. Rijnders

A. Spilt

F.W. Wittkämper

M.K. Zeeman

J.R. de Jong

P. Sminia

K. Franken 


\title{
Process Management and Quality Assurance for Intracranial Stereotactic Treatment
}

\author{
NEDERLANDSE COMMISSIE VOOR STRALINGSDOSIMETRIE \\ Report 25 of the Netherlands Commission on Radiation Dosimetry
}

This report was prepared by a subcommittee of the Netherlands Commission on Radiation Dosimetry (NCS), consisting of Belgian and Dutch scientists.

Members of the subcommittee Joep Hermans (representative NCS-board) Stan Heukelom (chairman) Nienke Hoffmans-Holtzer Hans Marijnissen An Nulens Geert Pittomvils Esther Raaijmakers

Dirk Verellen Sandra Vieira

NCS, Delft, the Netherlands

For more information on NCS Reports, see http://radiationdosimetry.org 


\section{Summary}

September 2006, the Netherlands Commission of Dosimetry (NCS) installed a subcommittee on quality assurance and quality control for intracranial stereotactic treatment. The idea behind this was setting up a report in which institutions with considerable knowledge on the subject share their experience with starters and users.

There were several reasons for the introduction of this report: 1) the development of dedicated stereotactic treatment devices, such as Gamma Knife, Cyberknife and Linac, the latter using add-ons like cones and MLC; 2) the rapid development of relocatable frames instead of invasive frames, allowing the introduction of fractionated stereotactic treatment; 3) the implementation of frameless stereotactic radiosurgery and radiotherapy in combination with imaged guided positioning technology like kV-imaging and cone beam CT; 4) the difficulties inherent in small field dosimetry; a proper understanding of do's and don'ts is essential; 5) the introduction of stereotactic treatment in hospitals as a standard technique "like any other".

The creation of the report was a long and complex process. Different technologies for intracranial stereotactic treatment as described above were already available or in fast development. In a rapidly evolving field as radiotherapy, considering only technology would outdate the report, even during the process of writing it. Therefore, we focussed on the critical question: what is the basic requirement for providing a safe and high-quality stereotactic treatment. Technology itself is not the only main quality assurance and quality control parameter. Department organisation, skills of users, treatment process structure, etc. have to be considered as well. Finally, nomenclature used in intracranial stereotactic treatment was and still is not always obvious, so definitions and descriptions should be established/specified.

These considerations crystallized into a report with starting point the process management to setup a new technology into a routine treatment facility as well as to retain it.

The report is set up in a modular way: the in- and excluded subjects of this report (chapter 1 ), a short history to introduce intracranial stereotactic treatment (chapter 2), the generic structure of the stereotactic treatment process (chapter 3 ) and generic advice based on that treatment process (chapter 4). Detailed advice is presented in additional chapters. With the setup of this report in this way each reader can easily find specific points of interest. In the 
digital version hyperlinks have been set to support that easy use. Of course: the novice in the field should start at the beginning!

Throughout the whole report the subcommittee has added "reflecting" points, indicated by the symbol:

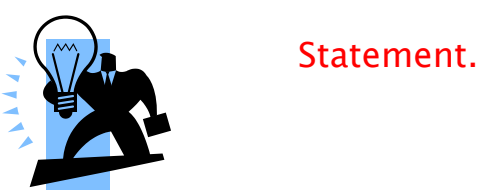

The content of those points might help the reader to reflect on important aspects of intracranial stereotactic treatment, both when starting or continuing an existing program in their department. As authors we would summarise our main message in the following two statements:

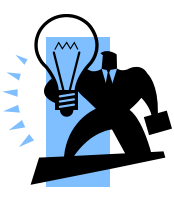

Statement 1.

Do not start or continue stereotactic radiosurgery (SRS) or radiotherapy (SRT) unless you have the capability, organisation and patient mix required for stereotactic treatment.

Statement 2 .

Stereotactic treatment has to fulfil quantitative quality and safety prerequisites, based on multidisciplinary risk analyses and setup conform current international standards for stereotactic treatment. 


\section{Contents}

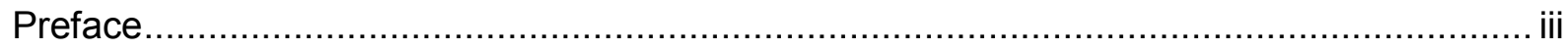

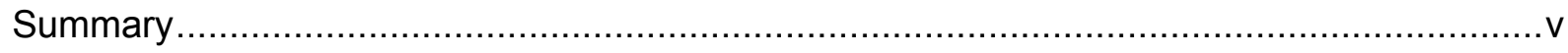

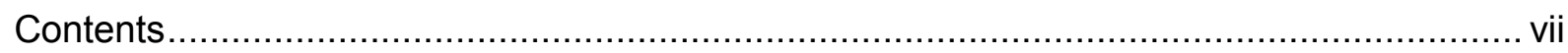

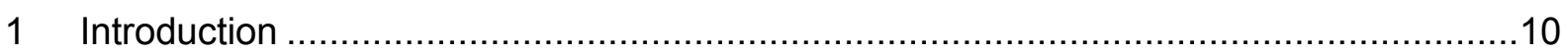

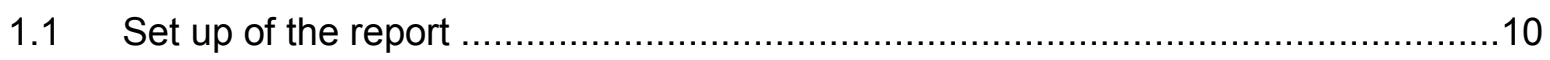

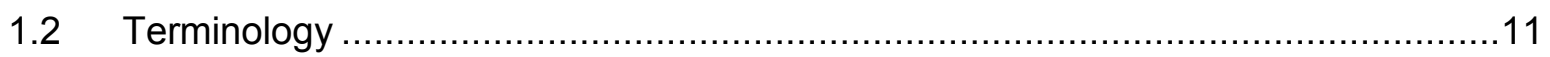

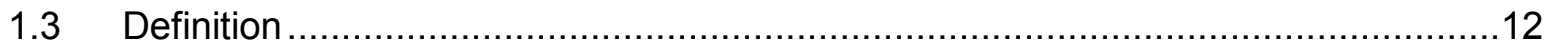

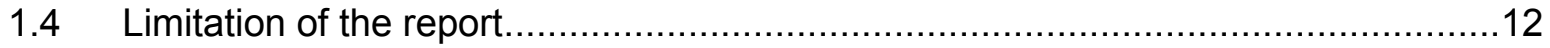

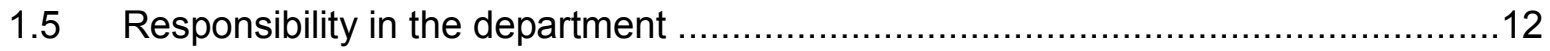

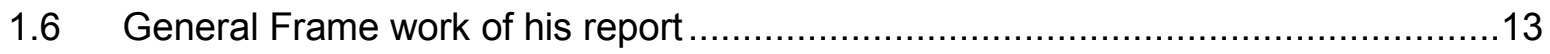

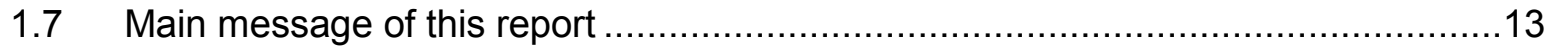

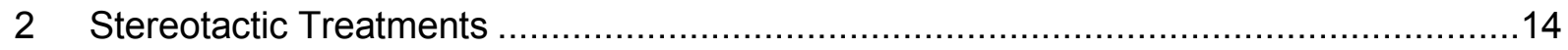

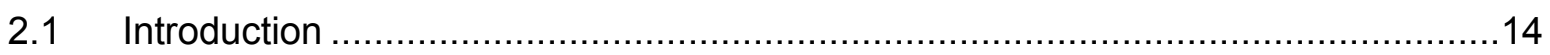

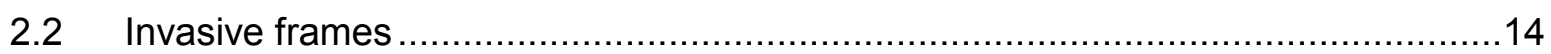

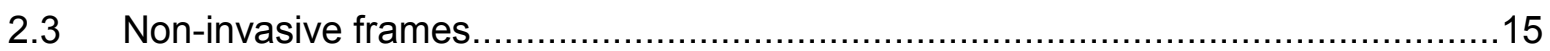

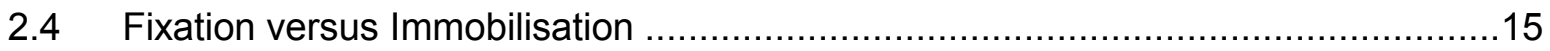

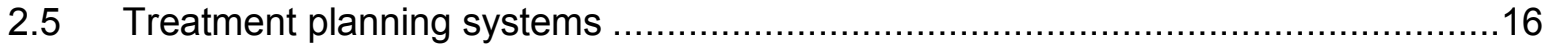

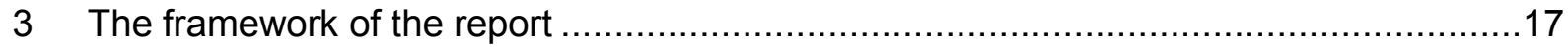

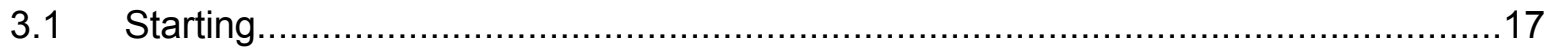

3.2 "How to setup, maintain and improve the quality of the stereotactic treatment" ......18

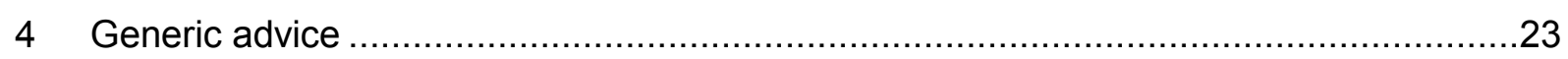

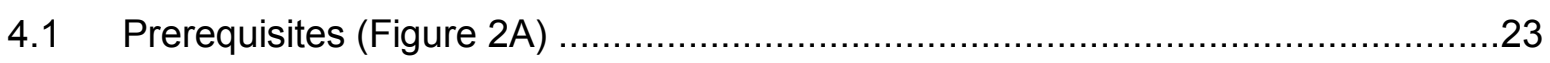

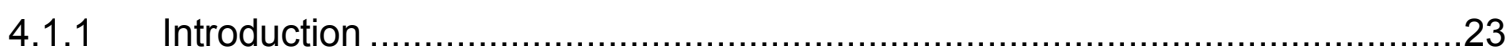

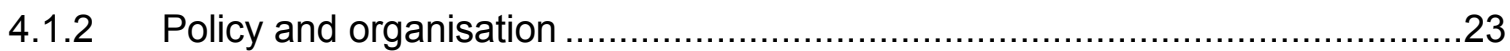

4.1.3 Knowledge \& Experience: competence of human resources .......................28

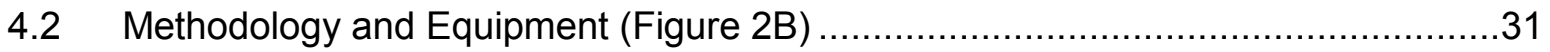

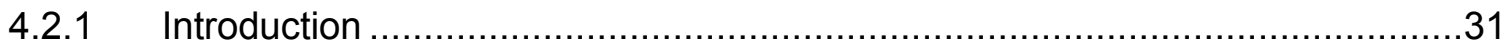

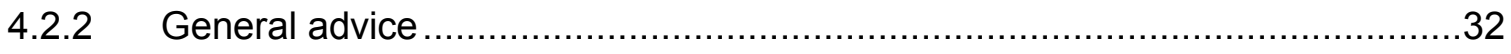

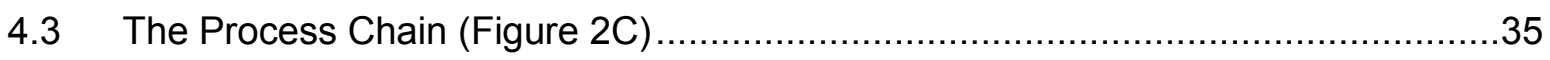

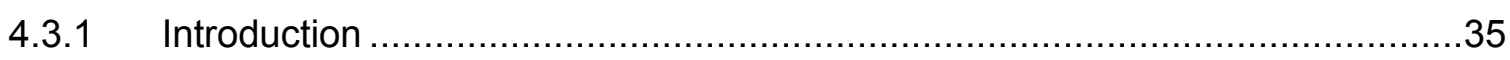

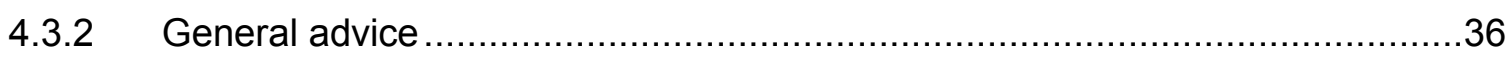

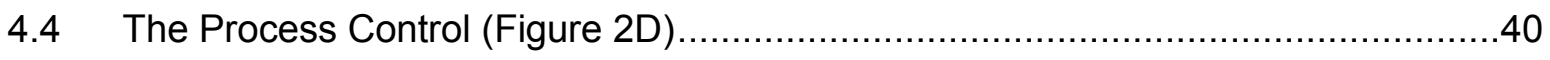

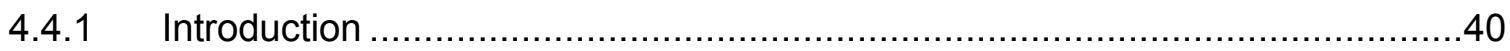

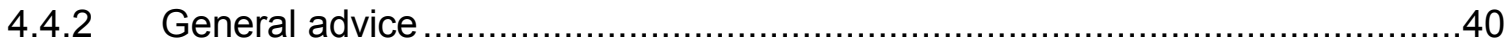




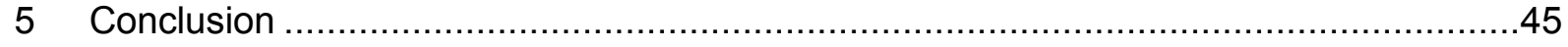

6 Appendix: Equipment and Methodology / Process chain ........................................47

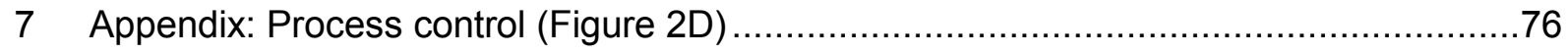

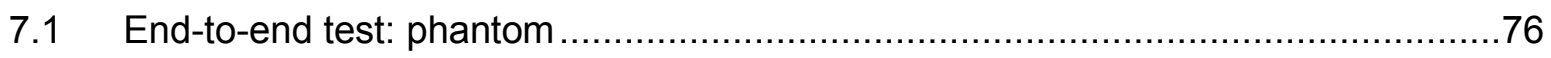

End-to-end test: dose detector ..................................................................

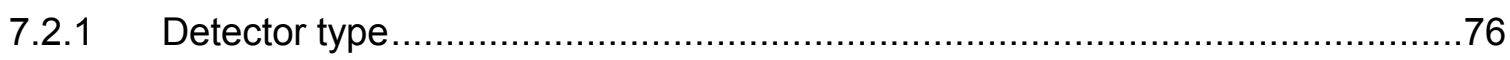

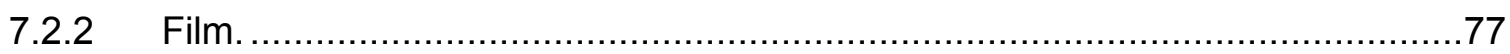

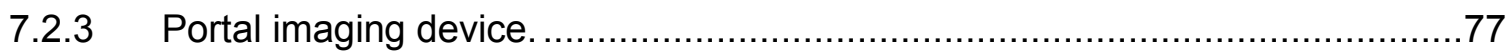

7.2.4 Pre-treatment verification: phantom.......................................................

7.2.5 Pre-treatment verification: detector........................................................

7.2.6 Pre-treatment verification: acting ..................................................... 77

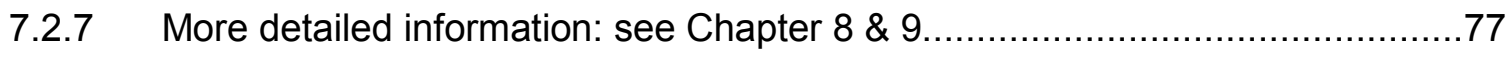

8 Appendix: Small field dosimetry: detectors ................................................... 78

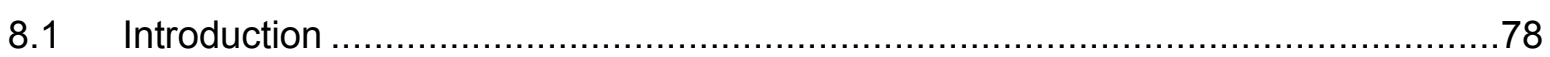

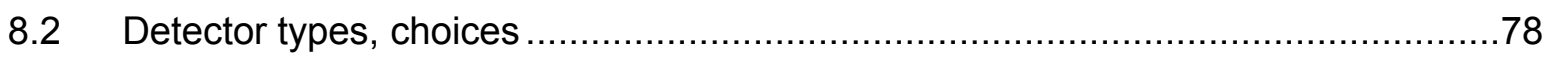

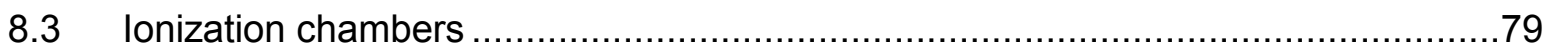

8.4 Diodes, MOSFETS and diamond detectors .......................................................

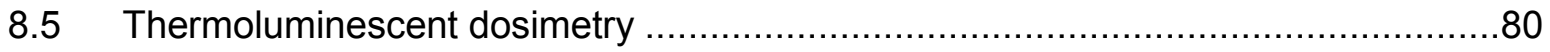

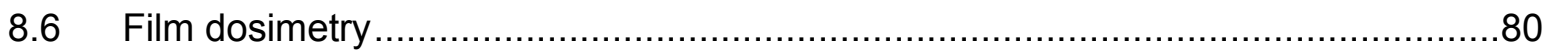

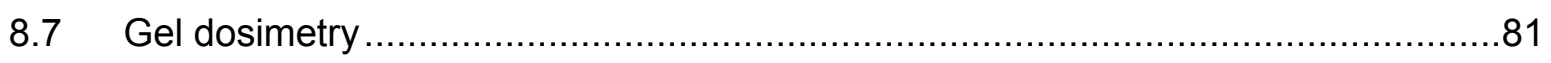

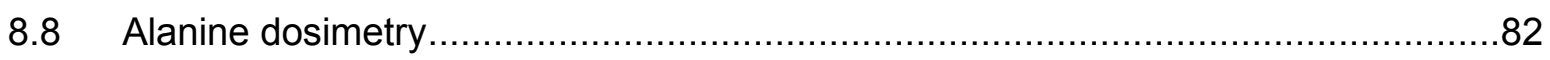

9 Appendix: Small field dosimetry: beam characteristics ....................................... 83

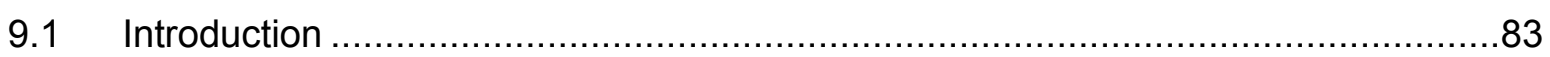

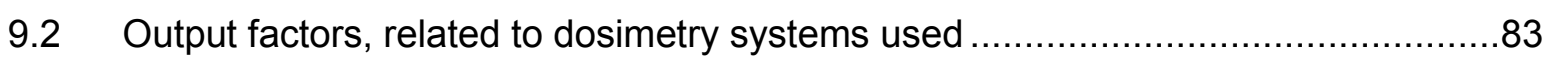

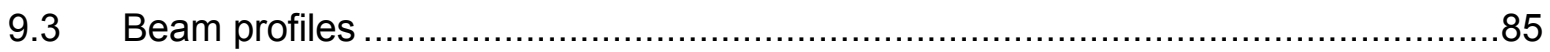

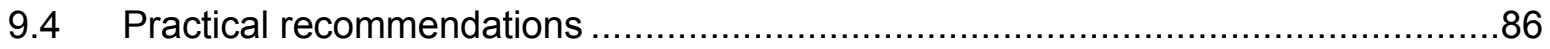

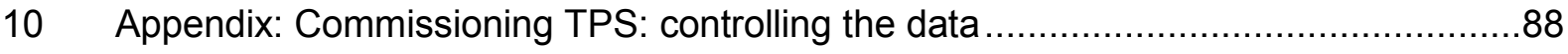

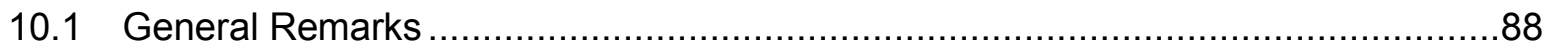

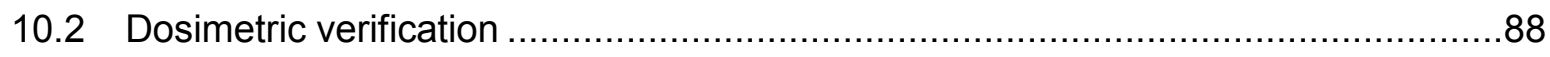

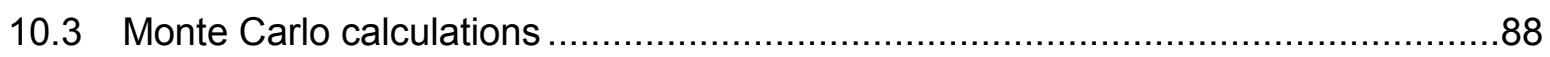

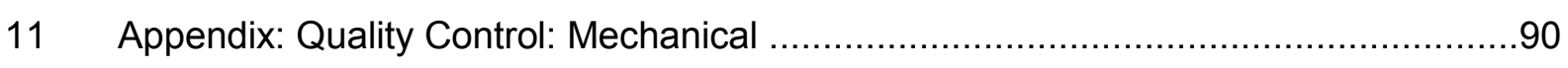

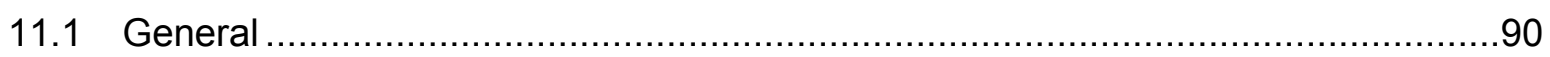

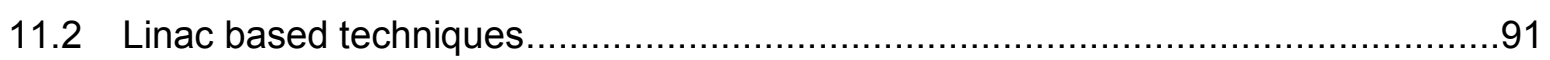

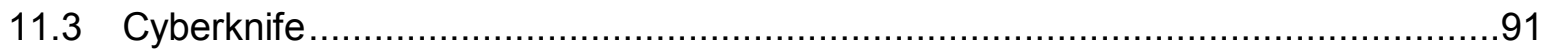

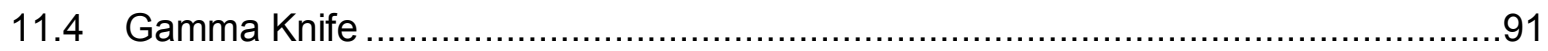




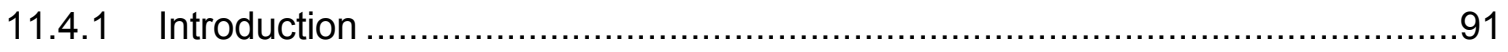

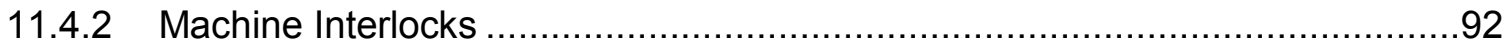

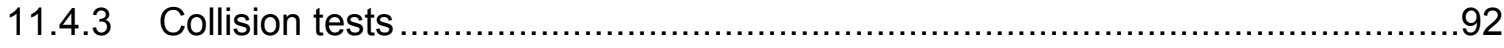

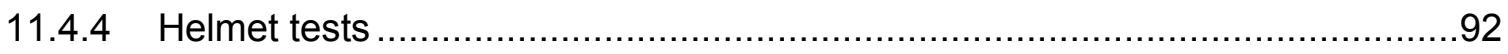

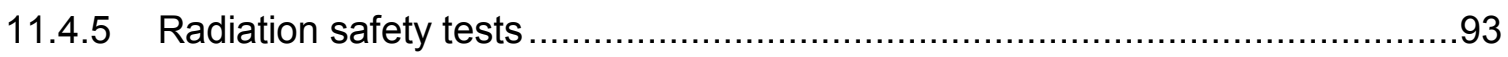

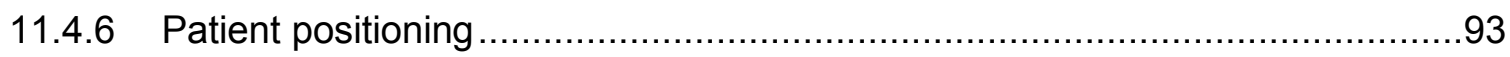

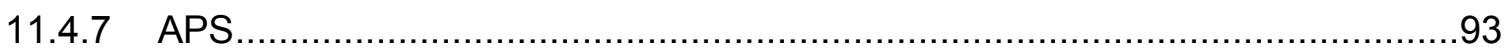

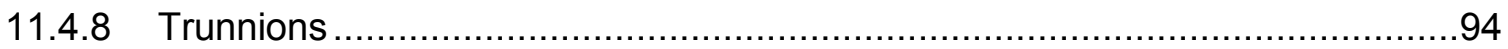

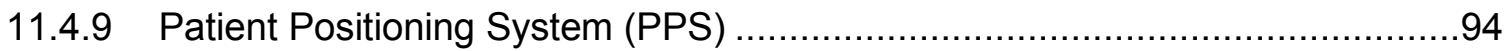

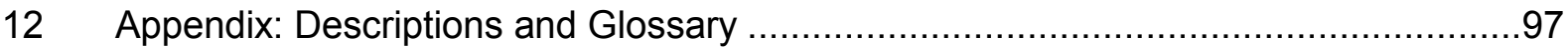

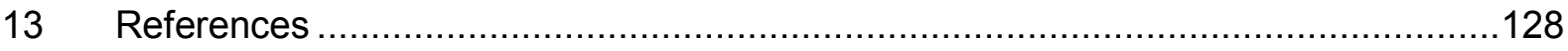

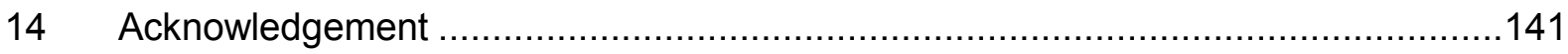




\section{Introduction}

\subsection{Set up of the report}

Intracranial stereotactic treatment is a high-fraction dose, high-precision technique based on the experience of several groups but lacking radiobiological evidence. Due to the high fraction dose, the highest possible accuracy in target positioning and dose delivery is required. High-level quality and safety assurance are, therefore, fundamental items in performing such a technique. To help institutions setting up a good quality assurance (QA) program making it possible to perform proper quality control (QC) for intracranial stereotactic radiotherapy, the Netherlands Commission of Dosimetry (NCS) installed a subcommittee in September 2006. This subcommittee consists of institutes with a considerable knowledge on the subject.

A lot has been already published about stereotactic treatment, both "intracranial" and "extracranial", containing mostly information about clinical setup and outcome, technology and quality and safety assurance elements. However, quality assurance is often reflected as quality control of technology. While information about implementing a new technology into a department to the level of a routine technique is scarce.

In the opinion of this subcommittee, departmental implementation and assuring stereotactic treatment as new routine technique is a complex process. Based on the many-year experience of several institutions regarding the dos and don'ts, the authors of this report choose to focus on "process management": both departmental management aspects to implement a new treatment technique as well as the treatment technique process itself, to ensure state-of-the-art treatments, i.e. safe treatments with a high quality. The reason is three-fold. First, choices made by the departmental management influence, in a positive or negative way, the quality and safety of a new treatment technique introduced in clinical use. Second, retrospective analysis of recent published incidents [e.g. 2, 9, 35] shows that the treatment process itself, e.g. procedures, is in most cases the main cause of accidents; while nowadays technology is more and more inherent safe. Finally, technology changes fast. Metaphorically speaking: technical advices for today will be out-dated the next morning. For those reasons the main title of the report "Quality assurance and quality control of intracranial stereotactic treatment" has been subtitled by "Process management and Treatment Technique". 
The report should incorporate all technology available, i.e. stereotactic treatments performed with conventional Linacs, the Gamma Knife and the Cyberknife. Furthermore, the report should be adjustable, reflecting changes in opinion and technology in due time. With the considerations above in mind the subcommittee decided to assemble this report in a modular way. It is structured as a long-term generic line regarding the subject with additional appendices (modules) containing more specific information. The latter can be updated and adjusted rapidly, driven by clinical relevant developments. Or easily added with supplements, for example, when institutes decide to use TomoTherapy for intracranial stereotactic treatment.

In the digital version of the report hyperlinks have been used supporting "shopping" in the report. Of course: the novice in the field should start at the beginning!

The report starts in chapter 2, with a short discussion about the original meaning of "stereotactic treatment". Chapter 3 describes the framework of the report, expressed in 5 pictures. Based on those pictures, chapter 4 gives generic advices, important to be considered. Chapter 5 gives conclusions. Chapter 6 to 11 contain appendices: more specific details about different subjects have been presented, most of them related to items reflected in chapter 4. Chapter 12 is an appendix presenting terminology used in this report. Chapter 13 contains literature references. Finally, in chapter 14 colleagues are acknowledged for their contribution on specific topics.

One should always realise that the report cannot be comprehensive in all its facets. The first reason is the "limited view" of the authors, reporting from their own experience. Despite the experience of the authors and the explicit effort to look at the whole process as good as possible, some aspects might be missed. The second reason is that changes in practice and techniques of stereotactic treatments will occur in due time.

\subsection{Terminology}

Terminology has been given in Chapter 12 "Descriptions and Glossary", based on the comprehension of the subcommittee. From here on in this paper, the first time a term is used in the report it is expressed in red and described in that chapter. 


\subsection{Definition}

Stereotactic treatment is used as a collective substitute for all high-fraction dose highprecision techniques, e.g. intra-cranial and extra-cranial stereotactic treatment. High-fraction dose is not defined, but in practice often a dose at least twice the conventional fractionation schedule ${ }^{1}$ of $2-3$ Gy is used. However, it is questionable whether "stereotactic treatment" is used correctly as synonym for all high-fraction dose high-accurate positioning treatments. Based on the meaning of "stereotactic" (see Chapter 2), only high-fraction dose highpositioning treatments "based on the best possible fixation" should be considered as stereotactic treatment.

\subsection{Limitation of the report}

This report only deals with stereotactic radiotherapy based on fixation, i.e. for which fixation is an important means to establish the required high precision in target positioning. Additionally, it includes frameless cranial stereotactic treatments as (non-invasive) fixation masks are used.

The report only describes aspects specific to reference frame based stereotactic treatments. It is assumed that the reader is familiar with $Q A$ and $Q C$ for conventional scheduled fractionated radiation techniques [e.g. 1, 56, 79, 84, 109, 128, 157] in all their aspects. Note that the term "frame" may refer to both invasive and relocatable frames and to non-invasive, external fixation techniques, such as masks. For cranial treatments, the difference in achieved precision and accuracy between fixation and "immobilisation" in combination with image-guided techniques may become negligible.

This report does not deal with so called "stereotactic body radiotherapy (SBRT)", where position verification on the treatment machine is only based on image guidance (IGRT) to get the required high geometric accuracy. The reason is that from a technical point of view a total different technology has to be considered, i.e. pre-planning and pre-treatment patient imaging [e.g. 157].

\subsection{Responsibility in the department}

In this whole report this subcommittee does not state who or which discipline (the radiation oncologists, medical physicists, engineers, radiation technologists etc...) is responsible for

\footnotetext{
1 As reflectance, Chapter 12 also gives the description of "accelerated fractionation", "hyperfractionation" and "hypo-fractionation".
} 
what, how and when. Assigning these responsibilities is very much dependent on the local organisational structure. However, assigning duties and responsibilities to people is one of the pillars supporting a good quality and a safe production process. Therefore, this subcommittee strongly advises organisations to do so explicitly and document it.

\subsection{General Frame work of his report}

Since the emphasis of the report is on the treatment process, it might in fact be useful for setting up any new radiotherapy treatment technique.

\subsection{Main message of this report}

Over the whole report the subcommittee has added "lighting points". The content of those points might help the reader to reflect on important aspects of intracranial stereotactic treatment. Both when starting or continuing it in their department.

As authors we would summarise our main message in the following two statements:

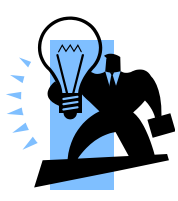

Statement 3.

Do not start or continue stereotactic radiosurgery (SRS) or radiotherapy (SRT) unless you have the capability, organisation and patient mix as required for stereotactic treatment.

Statement 4.

Stereotactic treatment has to fulfil quantitative quality and safety prerequisites, based on multidisciplinary risk analyses and setup conform current international standards for stereotactic treatment. 


\section{Stereotactic Treatments}

\subsection{Introduction}

The challenge in stereotactic treatment is to irradiate a target that is located near or is part of organs at risk. Examples of such targets include brain and spine lesions. Three aspects are typical for stereotactic treatment techniques:

1. A high-dose gradient between target volume and surrounding normal tissue.

2. A maximum accuracy in alignment of target and irradiation beam axis.

3. The highest dose gradient is typically nearest to the critical organ most at risk. This should explicitly be taken into account during treatment positioning

The high-dose gradient and high accuracy in target positioning are essential in order to give the target a curative dose, whilst simultaneously sparing the healthy tissue as much as possible. To achieve a high-dose gradient at the border of the target, it is crucial to allow for an extremely inhomogeneous dose distribution over the target. This means that it is not possible to fulfil the ICRU criteria for fractionated external beam treatment $[3,4,5]$. For example, the dose is prescribed at an isodose instead of a specific point. The choice of that isodose might be variable and physician-specific (Gamma Knife) or $80 \%$ isodose (Linac, Cyberknife based), on the condition that the maximum dose is somewhere in the target (GTV). The desired high geometrical accuracy in target positioning ensures both accurate dosing of the target and sparing of the healthy tissue. Nowadays sub-millimetre level, i.e. $\leq 1$ $\mathrm{mm}$, is pursued in positioning.

\subsection{Invasive frames}

"Stereotactic" has its roots in the Greek words "stereos" (three-dimensional, spatial) and "tactos" (ordered, arranged). Contraction of both words stands for three-dimensional arrangement or spatial orientation. Until recently, stereotactic radio surgery (SRS) and stereotactic treatment $[95,96]$ were associated only with the use of invasive frames attached to the skull of the patient for treating brain lesions. The frame is used as an orientation system during CT and MRI imaging, angiography and subsequent irradiation. Professor Lars Leksell and professor Børje Larsson introduced the use of such frames in radiosurgery in 1952 [88, 92, 94]. The success of these stereotactic radiosurgery treatments was based on a single high dose deposition with an overall three-dimensional precision of $1-2 \mathrm{~mm}$ obtained by using these stereotactic frames. Later on, invasive frames were replaced by relocatable 
systems, if possible, allowing fractionation of the treatment. Today, both frame systems are still in use.

The essence of a stereotactic frame is the rigid fixation of the reference frame (also named base-frame) to the patients skull, thus ensuring a "zero-point" for coordinate transformations of patient data, i.e. targets and organs at risk, over the whole treatment process. Specific auxiliary equipment connected to that reference frame used in all relevant imaging systems yields a correct and accurate correlation between target and organs at risk visualised with those imaging systems. Other auxiliary equipment can help for accurate target positioning on the treatment device.

\section{$2.3 \quad$ Non-invasive frames}

A drawback of invasive frames is that it is labour intensive and uncomfortable for the patient. Hence, techniques with invasive frames limit the irradiation treatment to a single fraction dose. To reduce the workload and increase the patients' comfort, non-invasive fixation techniques have been developed to simulate (or imitate) stereotactic frames. These techniques, however, do not fixate the head but merely immobilize it with a tendency to fixation [e.g. 66, 98, 153, 160]. The concept remains the same: to pin down the skull and with that the target, since its position has a strong relation to the skull. Similar considerations could apply to irradiation of a spinal lesion in which the target has a strong correlation with the spine itself. Example of a non-invasive modern device is the frameless system, in which strong immobilisation and image guided techniques are combined. Another non-invasive device is the relocatable frame, based on a mouth bite block and mechanical measurements whether or not the bite block is positioned well in the mouth. Nowadays the relocatable frame should be considered as out-dated.

\subsection{Fixation versus Immobilisation}

The use of a stereotactic frame in intracranial stereotactic treatment yields a strong fixation of the patient, i.e. target and organs at risk, on the positioning couch of all imaging and treatment devices used in the whole process. In this way intra- en inter-fraction uncertainties in patient positioning are avoided as much as possible.

Using a standard clinical mask is much less stable compared to stereotactic frames. In this report it will be mentioned as immobilisation. Using a standard clinical mask alone for stereotactic treatment is in general not advised. Only when using it in combination with high accurate on-line image guided radiotherapy, as for example cone beam CT, to guarantee 
exclusion of intra- and inter-fraction target movements, standard clinical mask systems might be applicable. To overcome on one side the negative aspect of invasive frame and on other side the non-fixation quality of standard clinical masks, a specific non-invasive mask system in combination with intra-fraction treatment position imaging is developed, to guarantee rigid positioning of the target during treatment as much as possible [e.g. 98, 153, 160].

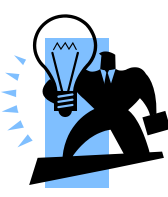

Statement 5.

To guarantee accuracy and stability of stereotactic frame systems, don't combine frame components made by different manufacturers.

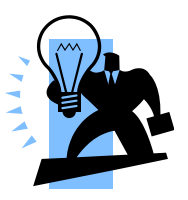

Statement 6.

Dedicated mask systems in combination with intra-fraction treatment positioning imaging should yield the same reproducibility as stereotactic frame systems.

\subsection{Treatment planning systems}

Of course, absolute dose and dose distributions have to be calculated with a modern dose treatment planning system. Herein, the stereotactic coordinate system is always used as the reference coordinate system. That means that a treatment planning systems should have the ability to recognise and handle such systems.

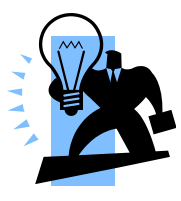

\section{Statement 7.}

Don't combine stereotactic frame components of different manufacturers. Treatment planning systems are not developed to handle such combined systems adequately and correctly in the stereotactic coordinate system used in the software. 


\section{The framework of the report}

\subsection{Starting}

Figure 1 presents the main elements that constitute the radiotherapy treatment process. The big arrow indicates the patient-treatment process chain, decomposed into its basic activities. Performance is only possible if the methodology and equipment is available to do so. However, additional prerequisites are essential to embed the performance into a departmental structure. A department quality assurance program is required to guarantee quality and safety in the initial task of the department: to treat patients and control that process.

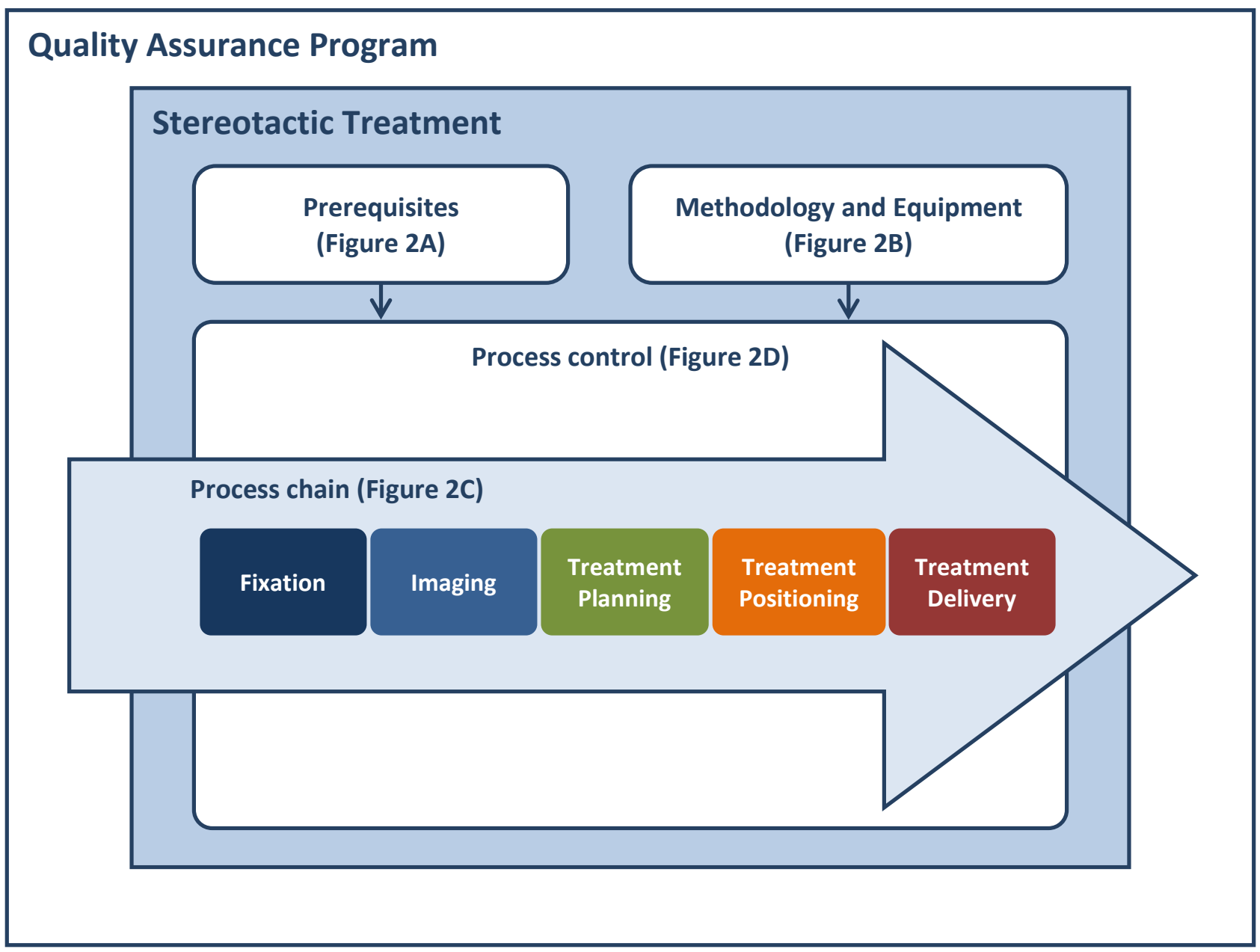

Figure 1. Elements of the radiotherapy treatment process. The figure is based on the original figure of Leer et al [91]

Of course, the importance of each element depends on target and radiation technique applied. For example, curative orthovoltage treatment and curative IMRT treatments require different efforts concerning QA and QC. High-fraction dose, high-precision techniques, such 
as stereotactic treatments, demand significant work to install and maintain a high level of QA and QC.

\section{2 "How to setup, maintain and improve the quality of the stereotactic treatment"}

Figure 1 relates to the framework of all RT techniques used on a routine bases. It can be used to setup, maintain and improve a new technique. Therefore, we decided to use Figure 1 as a reference guide for the frame work of this report (chapter 4 and later).

Achieving high quality and safety levels and avoiding errors in stereotactic treatments is a matter of a well-functioning organisation, the proper use of equipment and knowledge and skills of the users $[55,151]$. And, of course, the technical well-functioning of the equipment. However, with respect to the latter most modern equipment dedicated to stereotactic treatments already fulfil many of the technical quality and safety aspects. In addition, standard quality control activities already achieve mostly the required quality control levels.

Therefore, in this report we will focus on 4 elements mentioned in Figure 1, illustrated in more detail in Figure 2:

- Prerequisites (Fig 2A, \$4.2)

- Considering what is required before the technique can be implemented or what is needed to assure / improve the clinical routine with this technique;

- Equipment and Methodology (Fig 2B, §4.3)

- Considering the medical devices, methodology and software itself;

- Process Chain (Fig 2C, \$4.4)

- Discussing aspects of the treatment procedure itself;

- Process Control (Fig 2D, §4.5)

- Discussing aspects of quality and safety over the treatment process.

In the next chapter each element will be discussed in a generic way, guided by the aspects mentioned in the corresponding Figure 2. Details per aspect, if present, will be given in appendices. 


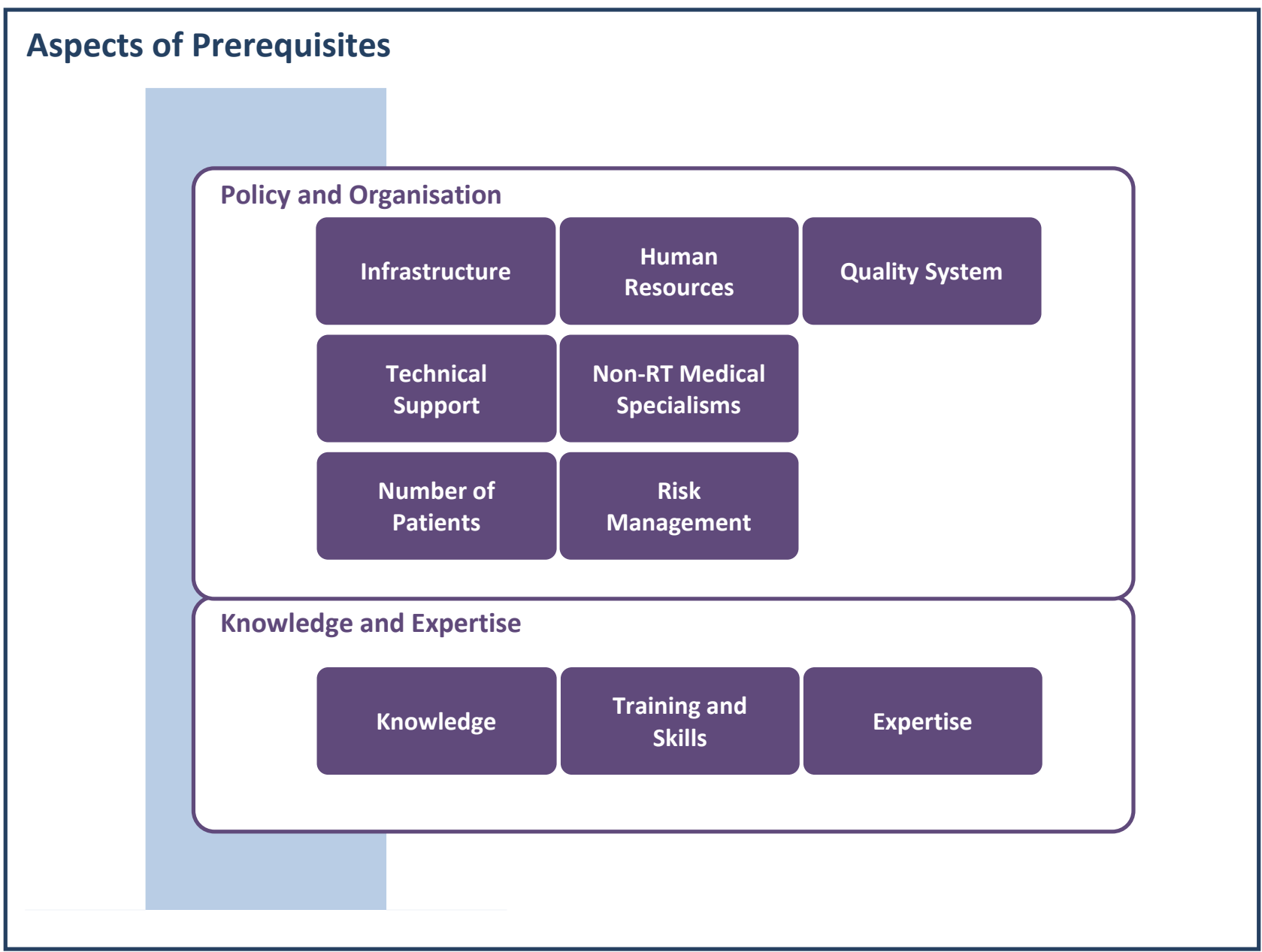

Figure 2A. Essential aspects in the organisation of stereotactic treatment in addition to conventional treatment techniques. 


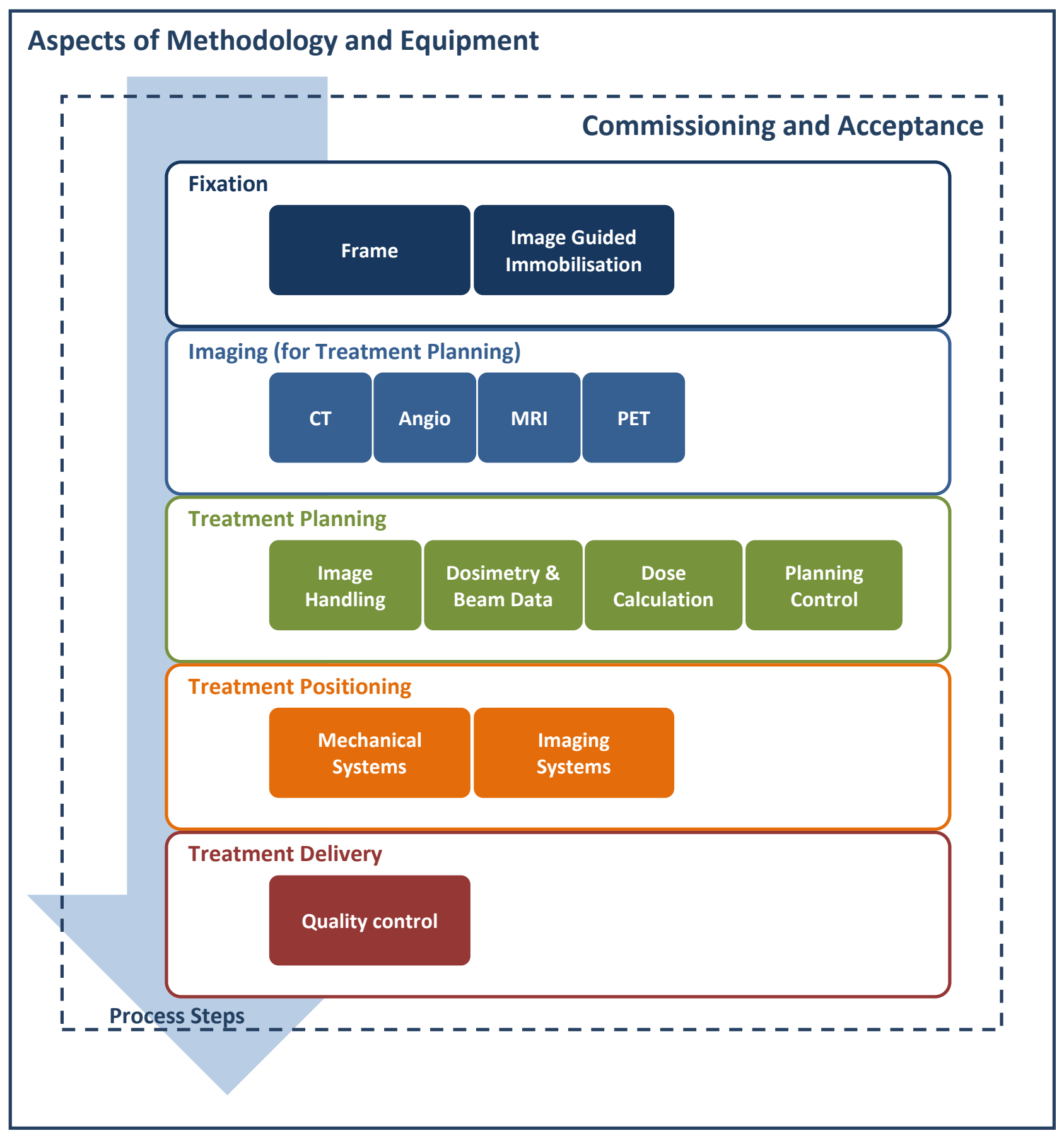

Figure 2B. Essential aspects with respect to equipment and methodology itself for stereotactic treatment compared to conventional treatment techniques. Chapter 6 contains detailed information about specific subjects. Note: the colour given to each aspect corresponds to that of the table given in Chapter 6 about specific details. To follow the hyperlinks relating the figure with Chapter 6 and vice versa, use the control key in combination with the left mouse button. 


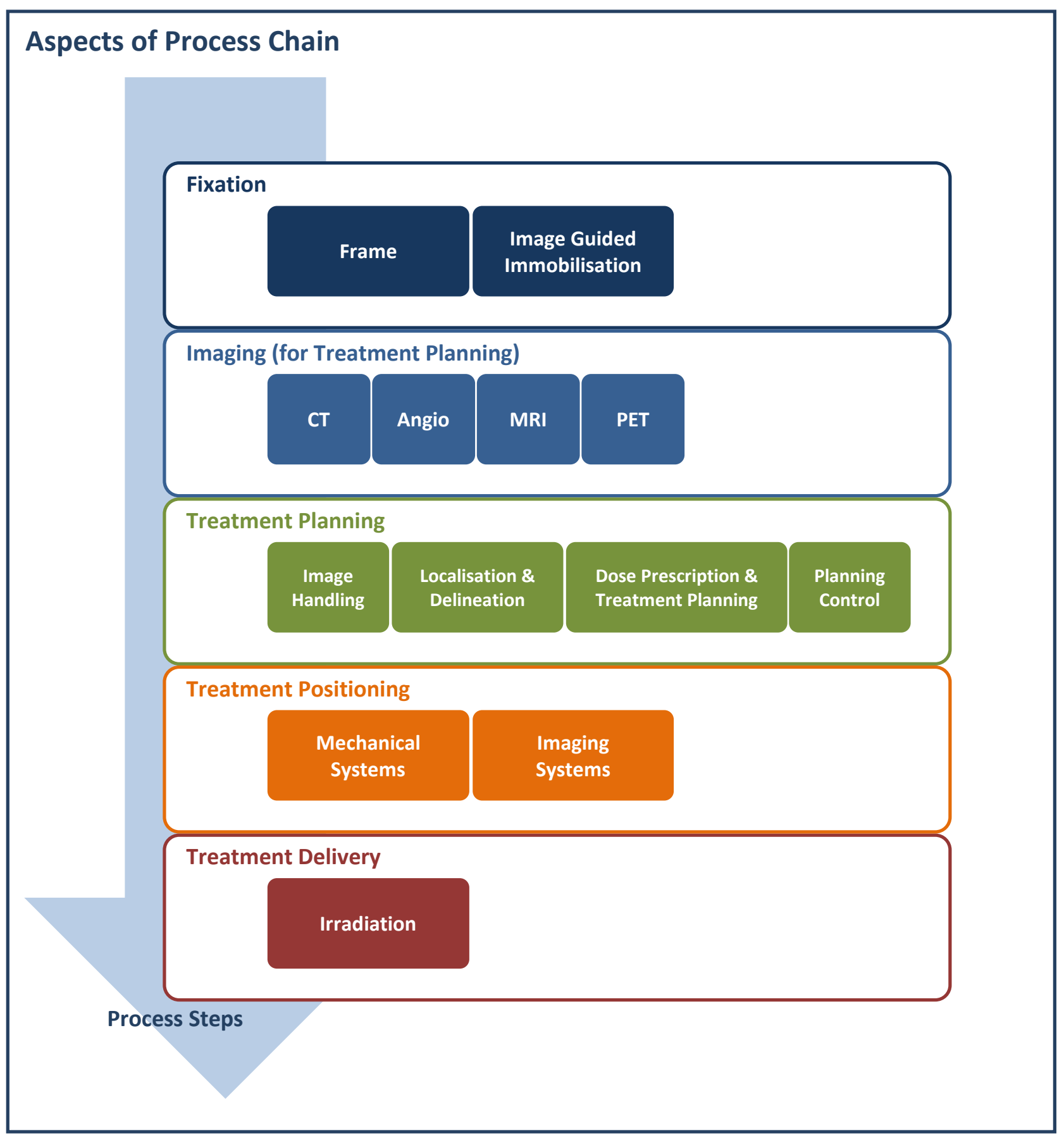

Figure 2C. Essential aspects with respect to the process chain for stereotactic treatment compared to conventional treatment techniques. Chapter 6 contains detailed information about specific subjects. Note: the colour given to each aspect corresponds to that of the table given in Chapter 6 about specific details. To follow the hyperlinks relating the figure with Chapter 6 and vice versa, use the control key in combination with the left mouse button. 


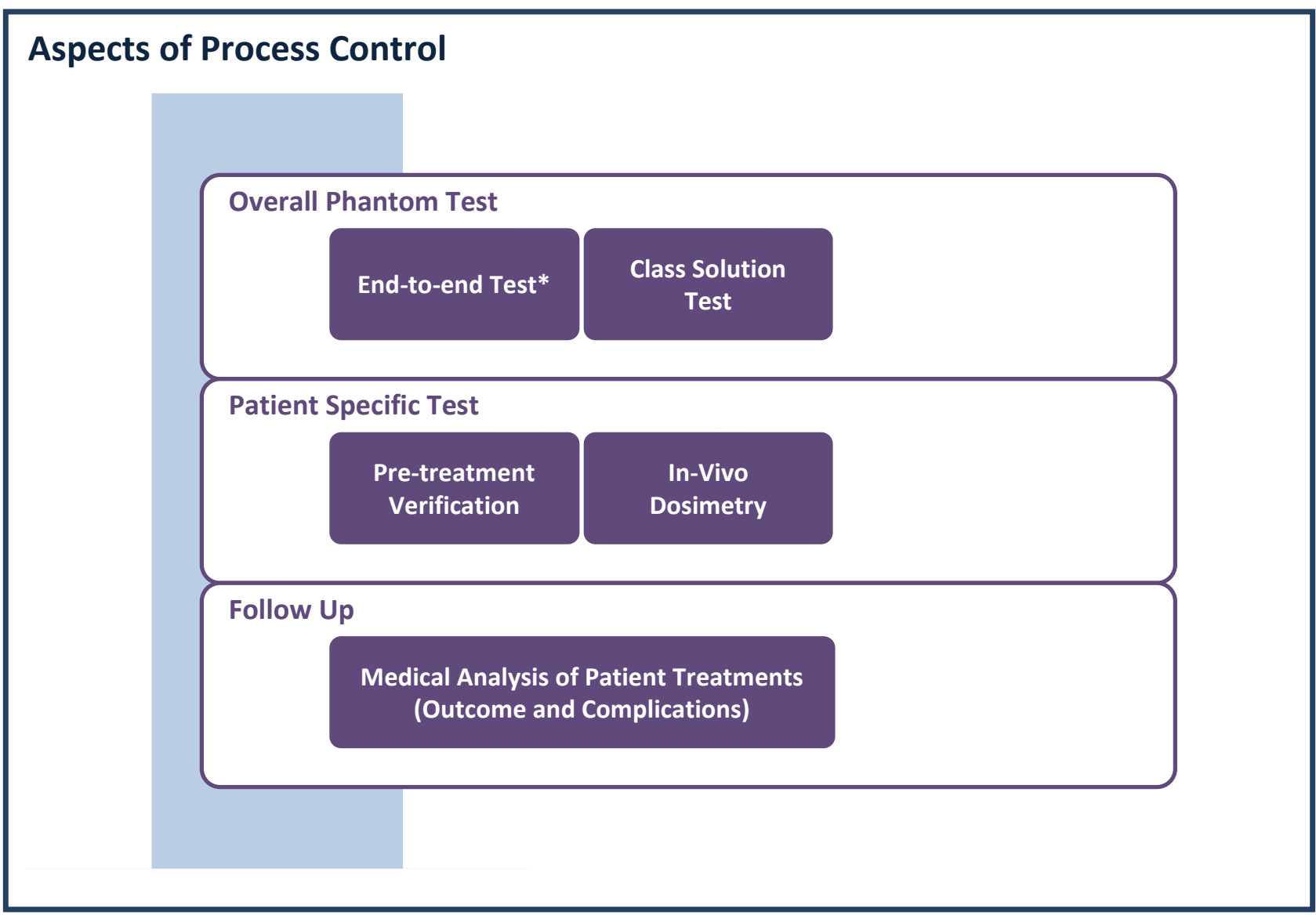

Figure 2D. Essential subjects in the process control for stereotactic treatment with respect to conventional treatment techniques. Chapter 7 contains detailed information about technical aspects of this specific subjects. Note *: specific details on the "End-to-end Test" can be found at Chapter 6 "Treatment Delivery, Quality Control \& Irradiation". 


\section{Generic advice}

\subsection{Prerequisites (Figure 2A)}

\subsubsection{Introduction}

When applying a high-fraction dose while high precision in target positioning is required, it should be realised that these techniques potentially have a much higher risk to seriously harm patients compared to standard techniques [57]. Awareness about the necessity of extra quality and safety levels should be present regarding the stereotactic treatment techniques applied, e.g. the use of invasive frames or a frameless system. Each technique requires a specific workflow, enough "critical mass" in number of patients and personnel as well as dedicated knowledge and skills of those involved in the stereotactic treatment process. Therefore, starting or continuing such techniques always requires a reflectance on policy \& organisation of the department as well as a reflectance on the required level of knowledge, skills and experience.

\section{Statement 8 . \\ It is advised to re-evaluate the prerequisites given in Figure $2 A$ on a regular basis.}

Some points discussed below may seem like stating the obvious. However, clinical practice shows that it is not $[55,70,151]$.

\subsubsection{Policy and organisation}

The aspect of the prerequisites relates to the infrastructure of the department. Although these aspects are important for conventional treatment techniques as well, for stereotactic treatment extra attention should be paid to the aspects summarised below.

\section{Infrastructure}

a. Straightforward process.

Technical or process workarounds should not be allowed. Transferring information, such as patient geometry obtained from different imaging systems, or data resulting from target segmentation and treatment planning should be 
unambiguous and straightforward. Both treatment planning system and treatment device should import all required information easily and unambiguously.

b. Dedicated equipment.

Use dedicated equipment that conforms to modern standards. Preferably, use only dedicated, validated and certified equipment with proven compatibility. It is strongly discouraged to adapt non-dedicated equipment for stereotactic use.

c. Take time to validate.

There should be ample time for thorough testing the equipment before treatment, both as a separate system and as part of the whole treatment chain. Make sure to test the equipment and workflow after updates, upgrades, replacements, repairs and process adjustments. The outcome and consequences of these tests should be documented properly, stating at least who tested what, when and why. This document should be a part of the stereotactic treatment quality system and can be called upon during an audit.

\section{Human resources: availability [e.g. 58]}

\section{a. Experienced personnel.}

Risk analyses of radiotherapy treatment processes learn that human factors, in particular inferior skills, are the main negative factors in accidents in radiotherapy. To avoid this as much as possible, all personnel should be well trained and focussed on their tasks. There is a need for redundancy in personnel for substitution and mutual reflection and discussions. Building up experience is key, and shifts in personnel should be limited as it is considered contra productive.

b. Workload.

Reducing the possibility to do severe harm by stereotactic treatment requires the highest focus of the personnel. Therefore, the workload related to the stereotactic treatment technique should not accumulate with already existing tasks and responsibilities. It is advised to provide sufficient staffing to ensure that all tasks defined in the process can be performed during normal working hours.

c. Teamwork.

The radiation oncologists, physicists, radiologists and personnel from other departments supporting the therapy should have clear responsibilities and duties during preparation and treatment execution. It is strongly advised to train the team and keep the formation stable over time, in order to gain as much experience as possible, as a team. 
d. Knowledge.

Each member of the team should have enough knowledge to consider all steps of the treatment critically and to intervene when mistakes are being made. The team members should have sufficient time to maintain and improve their level of expertise.

\section{Quality System}

a. Quality assurance system.

For both process and infrastructure, specific quality control indicators should be added compared to conventional treatments. An example of an additional process indicator is the end-to-end test (chain test) to assure the accuracy and robustness of the data transfer from CT scanner to the treatment device. An example of additional infrastructure quality check is the Winston Lutz test [132] to assure the correct alignment of the megavoltage beam isocentre with the mechanical isocentre. Another example of additional quality check is the quality assurance of immobilisation and target localization systems [142].

b. Protocols up to date.

It is recommended to develop a good protocol management and control system, ensuring up to date protocols.

c. Risk management.

Correct check and stop points at the right moments (time-put procedures) after and between process steps are highly recommended for stereotactic treatment. Develop the stereotactic treatment process as desired and perform a risk analysis on the intended treatment process $[7,8,11,62,69,76,141]$. What risks can be accepted cannot be described in general, since they may vary depending on the target site (lesion type) as on the local institutional situation (equipment, staff, etc.). However, the "ALARA principle" (As Low as Reasonably Achievable), well known and wide spread in the radiation protection domain, can be taken as a model. Finally, be transparent: stimulate comparisons with other departments, aiming to improve your own workflow.

d. End-to-end test.

The performance and analysis of the whole process on a phantom is complementary to a theoretical risk analysis. It is advised to do a dummy run and an end-to-end test not only before starting with (newly developed) stereotactic treatments but also on a regular basis, e.g. once a year. Note that the end-to-end 
test needs to generate end results, and should not be setup to identify specific problems as is the case for a quality control test.

e. Audit.

When starting stereotactic treatments, the teams experience is usually absent. It is advised to investigate what is present in (expert) institutions already performing stereotactic treatment and to learn from that. Furthermore, have yourself audited by external experts. This advice remains valid for departments for which stereotactic treatments have become routine.

f. Reflection.

Processes and habits do change [e.g. 39, 67, 110, 114, 133, 158]. Unnoticed and unilateral changes due to less alertness initiated by increasing experience are known to happen. They might weaken the safety of the treatment process. Therefore, reflect with colleagues the actual safety of the treatment process.

g. Changes in process components.

When a component of your process changes, e.g. introducing (a new) MRI in the treatment chain, start analysing the process from scratch. That seems timeconsuming but if the original process has been documented correctly, most unchanged steps can be checked quickly. Re-analysing the whole process reduces the chance of forgetting items in the process chain.

h. Changes in knowledge.

It is commonly accepted that the introduction of a new technique requires developing a database on treatment outcome data such as survival, toxicity, complications and safety notifications regarding treatments errors. Analysis of those data helps to adapt the process.

i. PDCA cycle ${ }^{2}$.

A quality assurance system should be a dynamic system as devices, process steps and experiences change. A dynamic quality assurance system requires the implementation of a Plan Do Check Act cycle. It is advised to apply this method..

\section{Technical Support}

a. Maintenance.

With the introduction of stereotactic treatment the technical support has to be adapted to the level required for those stereotactic devices. Eventually, the user is

\footnotetext{
2 e.g. http://www.isixsigma.com/dictionary/deming-cycle-pdca

e.g. http://asq.org/learn-about-quality/project-planning-tools/overview/pdca-cycle.html
} 
responsible for the local tolerance and action levels, these may differ between target sites. A risk analysis and consultation of other departments performing stereotactic treatments can help. Who is doing what needs to be agreed between department and manufacturer.

b. Information and Communication Technology (ICT).

The ICT infrastructure should support and facilitate robust data transfer and storage, as the stereotactic treatment should be straightforward. Usually, computer network support is delegated to another department in the hospital. Therefore, good service level agreements should be made regarding the reliability of the network and guaranties of immediate assistance when needed. The computer network department should know the consequences of improper functioning of the ICT facilities.

\section{Non-RT Medical Specialisms}

a. Interdepartmental relationships: medical support. Localising the target volume frequently requires the expertise from other medical specialities. In general, those specialities belong to different departments with their own processes and quality control and quality assurance system of medical devices. Therefore, it is advised to maintain a sustainable relation with those departments and to develop a mutual interest in setting up protocols for and education of the personnel of those departments. This may help to obtain an effective and efficient contribution from those departments.

b. Commitment: technical support.

It is recommended to persuade the involved departments to agree to additional quality control and quality assurance measures of the medical systems, in order to fulfil the technical requirements of those systems for a correct and safe stereotactic treatment. For instance, this comprehends informing your department on planned and unplanned upgrades and changes in equipment and/or software.

\section{Statement 9 .}

Make arrangements with other departments to use their equipment and personnel for quality control phantom measurements. 


\section{Number of patients}

a. From complex to routine.

Each new irradiation technique should shake off the predicate "experimental" or "new" technique in time. For stereotactic treatment, this means it should become a "complex routine technique", smoothly integrated in the departmental patient flow.

b. Good practice.

Paper and phantom is not enough to create and maintain an adequate level of well-trained and experienced personnel. A sufficient number of patients is required to learn from complicated situations and medical outcome. The minimum amount of patients per person, per discipline for proper stereotactic treatment is not described in literature yet. In the next statement below we give a guide as advice. However, in clinical practice this is not always achievable. If this condition cannot be met, the department is responsible to gain experiences by other means, e.g. rehearsal dummy runs, multidisciplinary continuous education, hands-on training.

c. Interchange ability.

Experience built up in one stereotactic treatment technique will not always be valuable in another technique. Therefore, when implementing a new technique make sure to evaluate it and start from scratch again, if needed.

7. Risk management $[7,8,11,62,69,76,141,164]$

a. Patient \& Organisation.

Risk analysis, as a tool to investigate the treatment process, can also be used to evaluate the risk-levels for a patient and to find the weak links of the departmental organisation. Realize that a process aspect can introduce a high risk for severe patient damage while it is a low risk point from the organisation point of view. And vice versa. It is therefore advised to consider both patient and organisation.

b. Multidisciplinary.

Multidisciplinary risk analysis is a prerequisite for a good analysis. Discuss the outcome with less involved colleagues of the department or from other departments / organisations.

\subsubsection{Knowledge \& Experience: competence of human resources}

Beside availability of human resources, the level of education, training and experience within the team should be guaranteed. These aspects are even more important for the 
reliable execution of a stereotactic treatment, compared to conventional treatment techniques. Concerning the "learning processes" [e.g. 53, 86] this means that the knowledge and skills of the personnel need to evolve from "unconscious incompetent" to "unconscious competent"3. An important prerequisite is to safeguard continuous development of these items for the whole team.

\section{Knowledge (from "unconscious" to "conscious")}

a. Passive.

Although "passive" in action, the first and indispensable step to obtain knowledge is to study the literature, attend (manufacturer / vendor) courses and visit experienced institutions.

b. Active.

Education learns that the best way to acquire a fluent knowledge is to practice that knowledge, for example by giving presentations to colleagues and others interested in the subject. Giving presentations requires summarising the essentials in the gained knowledge, thereby rehearsing the most important aspects of the subject.

\section{Training \& Skills (from "incompetent" to "competent").}

a. Be competent.

Beside the required knowledge, adequate training is essential before starting.

During the training, the slogan should be "a stupid question doesn't exist".

Training means developing skills by executing parts of the treatment chain and by performing the whole procedure on a phantom repeatedly ("dummy runs"). It is important to include variations and apply increasing complexity, such as irradiating two or three target volumes simultaneously.

b. Hands-on.

In addition, acquiring hands-on experience in 'experienced' centres should give valuable insight.

\section{Expertise}

a. Complex but routine. (to "unconscious competent")

\footnotetext{
${ }^{3}$ According to literature on "learning", the stages in acquiring skills follow the cycle "awareness learning - practice" in which the level changes according to the cycle "unconscious incompetent conscious incompetent - conscious competent - unconscious competent".

[e.g. https://en.wikipedia.org/wiki/Four_stages_of_competence]
} 
As stated above in the section "policy \& organisation, number of patients", stereotactic treatment of patients should become a "complex but routine" patient treatment technique. To achieve the required level of experience, the number of patients per person per discipline should be high enough.

b. Continuation.

Initially, all the team members are involved in the whole treatment chain. The evolution to a "complex but routine" technique often results in an increasing team size due to an increasing number of patients. In turn, the larger team will lead to specialisation, possibly at the cost of knowledge and skills required by other subgroups. Regular practical training programs help to keep the overall and specific expertise of all team members. Such training is strongly advised. A record should be kept stating who followed the training and when.

Statement 10.

Building up and maintaining a good level of competence and routine, team members have to practice patient treatment in a sufficient amount. Some guidelines:

1. With 25 patients or more equally distributed over a year, competence and routine will be established; extra training is not required.

2. Less than 10 patients per year we advise not to implement stereotactic intracranial treatment; competence and routine will not (easily) be build up and maintained.

3. Between 10 and 25 patients per year the department is advised to setup an additional internal training program.

4. Be careful to consider all intracranial stereotactic treatments as "a single group of treatments": specific lesions might require specific competence.

These guidelines provide a way of thinking, they are not a code of practice. 


\subsection{Methodology and Equipment (Figure 2B)}

\subsubsection{Introduction}

Institutes that start with stereotactic treatments usually already have the treatment device(s) and treatment planning system with which they intent to start this technique. In general, the equipment determines a first way of thinking on how to introduce it at the department. The starting point seems to be cost-effectiveness on the one hand and acceptability of present accuracy levels of those treatment devices and planning software on the other hand. The drawback can be that the desire to start-up stereotactic treatment under these conditions can lead to undesirable concessions to accuracy and safety.

However, there are better starting points from quality and safety's point of view. Firstly, the question should be what level of accuracy in dose delivery to the patient is required and how can this be obtained. This implicates that one should start by making a minimum requirement list as if it were a new to build department, and then compare that list to the equipment in place. This means that the user starts from scratch to investigate what stereotactic treatment devices are needed and what the accuracy requirements are [e.g. 47, 22, 84, 107]. Secondly, a good insight is required how (non-)well working devices and auxiliaries can influence the quality and safety of the whole treatment chain. Error reports about inaccurate treatments support this [e.g. 2, 9, 35]. After that, the equipment demands can be compared to equipment already available in the institution.

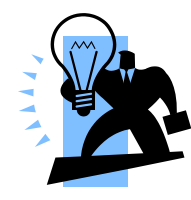

\section{Statements 11 .}

- The total dosimetrical and geometrical uncertainty of the medical equipment and software should be evaluated with an "end-to-end" phantom test.

- It is the task of the department to assure that the dosimetrical and geometrical uncertainty on a phantom is within the accuracy limits stated by the department.

- Accuracy limits stated by the department should be in line with commonly accepted criteria or supported by common experience.

When setting up guidelines to ascertain a safe introduction of high-quality stereotactic treatment, we impulsively look at critical aspects of available hard- and software. However, 
such criteria will become out-of-date shortly after publishing such a guideline. In addition, the composite effect of uncertainties in the underlying components on the accuracy and safety of the patient treatment is hardly predictable. Therefore, we chose to focus on the total uncertainty in whole treatment chain, i.e. dose delivery to the target volume and organs at risk.

\subsubsection{General advice}

Since compatibility of medical devices is a weak point, we present in this paragraph "general words of advice" about equipment. In addition, in Chapter 6 we give some relevant detailed information regarding equipment used for stereotactic treatment.

\section{Compatibility.}

Data transfer between diagnostic devices, treatment planning system and irradiation device should be unambiguous. This data transfer incorporates patient geometry, information about fixation and patient (re)positioning control. All devices should correctly deliver, handle and interpret this information. Yet nowadays, there are many pitfalls, even if manufacturers state that compatibility should be "no problem". Assure the correct detection in the treatment planning software of the invasive frame or the frameless marker based system and the accurate translation of this data for positioning the patient on the treatment machine.

\section{Single manufacturer.}

The easiest and best way to attain compatibility between al devices is to use equipment from a single manufacturer-vendor. This should provide the best guarantee that all devices and are tuned to each other. CE-marking ${ }^{4}$ might help. Still, this does not discharge the team from performing a risk analysis, testing equipment and executing dummy runs.

For the Gamma Knife $®$ and the Cyberknife $®$ Robotic Radiosurgery system the single manufacturer-vendor concept seems without question as the irradiation equipment is supplied both with an integrated treatment planning system and with auxiliaries to practice stereotactic treatment.

For Linac based stereotactic treatment equipment the single manufacturer-vendor philosophy is not the only option. In general it is accepted to configure an irradiation facility based on devices supplied by different manufactures. Compatibility is then a main

\footnotetext{
${ }^{4}$ CE-marking (Conformité Européenne): the European Directive about quality mark related to wellbuilding of medical equipment.
} 
issue, often requiring additional quality control and allowing a more error-prone process. However, a dedicated planning system for stereotactic treatments is preferable as it is built on issues of stereotactic treatment safety and on the possibility of tuning the treatment planning algorithm for small fields without compromising the accuracy of large fields modelling.

\section{Second best.}

A second best solution is a clear and well working cooperation between manufacturers of different devices and suppliers. Of course, having more manufacturers will result in less tuning, while complexity and risks increase exponentially with the number of manufacturers. Therefore, if devices of different manufacturers are used, the risk of treatment errors, caused by mismatching devices, should be analysed explicitly. Realise that a single vendor selling such multi-manufacturer stereotactic system is not an guarantee for compatibility of devices.

\section{Black box.}

Do not handle the medical equipment as a "black box". The operators can usually understand the way the devices and software work through experimentation and by asking the vendor for explanation. This knowledge helps to control and manage the systems. Additionally, it avoids "blind testing", i.e. performing tests and checks, e.g. based on literature, without a proper understanding of the underlying rationale.

\section{Quality Control.}

Most quality control tools to measure the actual accuracy levels of equipment are wellknown techniques. They are many QA/QC reports and manufacturer-specific protocols describing relevant tests. However, the required accuracy levels and test frequencies may change in time. Moreover, new equipment may need additional tests or may make existing tests obsolete. It is vital to understand the equipment and thereby know the required QA procedures. A proper risk analysis regarding the operation of the equipment helps to identify weak links [e.g. 66, 98, 143, 153, 160].

6. “Add-ons".

"Add-on" systems usually pose additional uncertainties in the treatment procedure, possibly causing treatment errors. How to deal with it depends on the amount of integration in the basic equipment system. For Linacs the policy should be to avoid such system whenever possible. An example is the "add-on" multi-leaf collimator (MLC) as they are now succeeded by accelerators with build in MLC systems having the same or even better spatial resolution. In contrast, for several Linac irradiation techniques add-on cones are the best solution to create desired irradiation fields. For Cyberknife system and 
Gamma Knife system add-ons are much more integrated in the basic system. E.g. the Iris Variable Aperture Collimator or Incise Multileaf Collimator (MLC) for the Cyberknife system and the aperture system of the Gamma Knife. For those specific add-on systems outlining of the add-ons to the irradiation source system is more guaranteed . Overall: in all cases one has to balance the (dis)advantages of add-on systems.

\section{Acceptance and commissioning}

Devices used in stereotactic treatment have to be accepted and commissioned before they can be used correctly and safely in clinical routine. Acceptance and commissioning are the responsibility of the department.

Acceptance means that the department takes over the responsibility from the manufacturer for the functioning of the equipment within specifications agreed upon between manufacturer and department. It also means that it is up to the department if stronger accuracy levels than specified are required. Hence, state and agree in advance with the manufacturer on the specifications required Commissioning is gathering information about the working of a device in the clinically used range. It is a task to perform after acceptance of that device. It means that when commissioning shows that adjustment of a device is required to fulfil specifications, this adjustment has to be considered as an update / upgrade and in fact the machine has to be accepted again. So, state and agree with the manufacturer clearly on what is acceptance and what commissioning. 


\subsection{The Process Chain (Figure 2C)}

\subsubsection{Introduction}

The process chain for stereotactic treatment is in essence not different from conventional radiotherapy (Figure 1). Fulfilling the prerequisites (Figure 2A) and having adequate methodology and equipment provides the basis of the safety and the quality assurance of the stereotactic treatment. Previously we already stated that the process chain should be straightforward. In this section, we discuss the process chain in more detail.

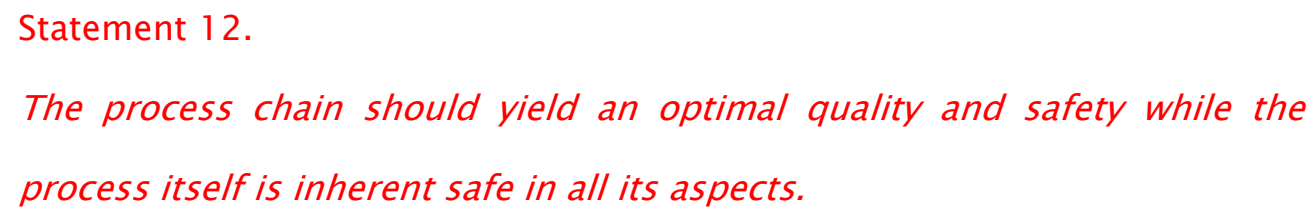

Balancing quality and safety against efficiency and cost-effectiveness provides the optimal result. A risk management system, incorporating a well-established "plan-do-check-act" cycle is essential to achieve this. The institute has to check their "optimal quality and safety" levels against other institutions on a regular basis. A "100\%" safety level can never been achieved, but considering and analysing several aspects of the process chain in more detail can be helpful to optimise the chain result as much as possible.

\section{Statement 13.}

Realize: fixation to the treatment couch may become unsafe if the patient experiences an unexpected dangerous physical response, e.g. coughing, vomiting or having an epileptic attack. People performing the patient treatment should be trained to react appropriately in those situations! 


\subsubsection{General advice}

In this paragraph we present staccato "general words of advice", based on our experience setting up a good process chain. Chapter 6 contains more detailed advice regarding the process chain information, specifically related to stereotactic equipment.

\section{Process chain description (Figure $1 \& 2 C$ )}

a. Describe "on paper" the main process steps (e.g. Figure $2 C$ ) and sub-steps per main step.

b. Secure such a process with adequate measures, i.e. management-wise (process) as well as facility-wise (equipment).

c. Perform, on a regular basis, the "plan-do-check-act" (PDCA) quality control cycle over the whole process or over sub-parts. In the latter situation mutual relationships between process (sub)steps should not be ignored.

d. To support aspects b and c, appoint a process-owner for each main process step. These persons are responsible for managing and facilitating their part while maintaining and improving its quality and safety within the whole process. This management and facilitation have to be based on the contents presented under Figure $2 A$ and Figure $2 B$.

\section{Quality assurance of a process step (Figure 3)}

a. Describe for each process step the inquiry, the activity to be performed, the resources required, the product that would be obtained and its quality parameters, changes in resources if expected, the quality control process on product and resources and, finally, what to do if the inquiry is not fulfilled. ${ }^{5}$

b. "The devil is in the detail" has two faces. On one hand: don't lose yourself into details; i.e. limit such descriptions to main points / aspects in the treatment process chain. On the other hand: recognize important details; i.e. they can break down your process into a dangerous process.

\section{Inquiry and Resources (Figure 3)}

a. Describe the inquiry and the quality parameters of the product.

\footnotetext{
${ }^{5}$ As an (incomplete !) example (see Figure 3). Inquiry: generate CT patient data. Specific process: CTscan. Product with quality parameters: CT information of that patient with $1 \mathrm{~mm}$ coupe distance and horizontal alignment of horizontal laser markers. Resources before: patient data and lesion site. Resources after: patient data with information about actual suppliers used during the scan. Quality control on the quality parameters: the desired product is generated. If not, the scan is repeated, until satisfied to the quality parameters.
} 
b. Describe the resources that have to be used. For example: protocol, database information. But also: human skills to perform that process step.

\section{Product and Resources* (Figure 3)}

a. Describe the resources after use. Sometimes they change due to the process performed. For example: patient database information about the progress of his / her treatment process.

b. Describe checklists to guarantee the correctness of the proceeding activity and (expected) resource changes.

c. Realise that setting up a good check is not easy, irrespective of the institutional way of working and requirements. When designing a check, one should keep the following in mind:

i. A check should warn about a possible mistake. This seems like stating the obvious but in practice it is rather difficult to achieve this.

ii. The check should be independent of the way activities are performed in the process. For instance, a check based on repeating the activity as a check is hardly an independent check.

iii. Checks should be performed by independent and qualified checkers.

iv. Checks should be implemented on the appropriate level and moment in the process chain.

v. To summarize: a check should be useful, sensitive and specific.

vi. Here again: "the devil is in the detail".

d. If the output of an activity cannot be checked easily in the next process (sub)step, a check on the whole process chain should be made. For example, to check the actual radiation field against the planned radiation field (just) before dose delivery.

e. Realize:

i. In general, the final product of one (sub)step is the input for one or more succeeding (sub)steps.

ii. Some (sub)steps require no input at all. In a similar way, the input of a specific process might be the final product of multiple process steps.

\section{Statement 14.}

"The devil is in the detail": be aware that work process and culture in a department are main factors to influence quality and safety. 


\section{Quality Assurance of a Specific Process}

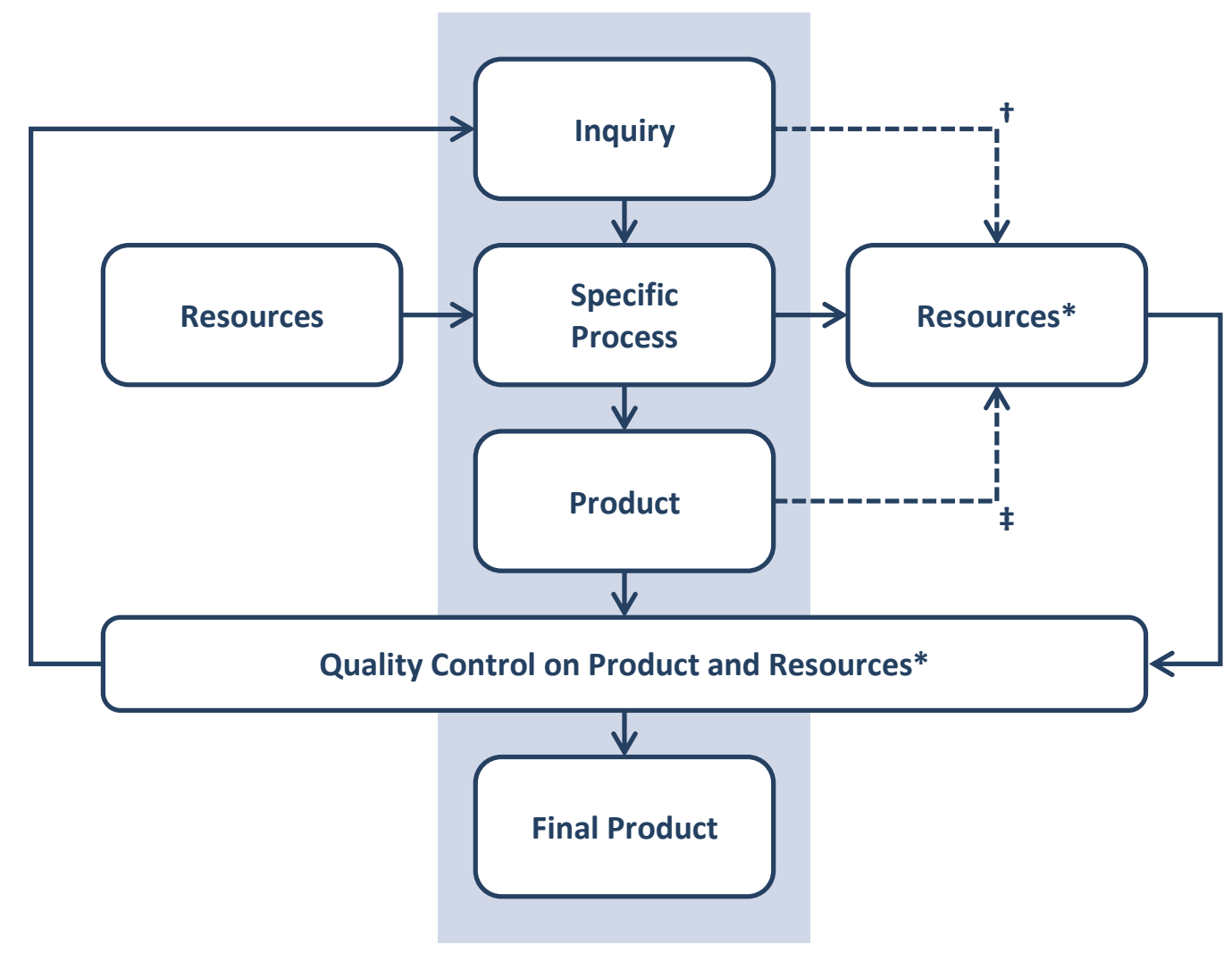

Figure 3. Quality assurance about a specific step in a process. The light-blue bar indicates that specific process. For instance, the creation of a patient CT dataset, or the calculation of a dose distribution for an individual patient. Resources indicates the supplies to perform the process; due to that process they may change! In general, the product itself and the change in resources ( $t$ ) are predictable so they can be checked on outcome ( $f)$. If the outcome is as expected, the final product is delivered. Realize that a final product might be one of the resources for the next process step. Figure 3 is based on the "plan-do-check-act" cycle.

\section{Dealing with failures in sub-steps (Figure 3)}

a. Describe for each (sub)step what to do if the activity in the process (sub)step cannot be performed, for example, due to incomplete input or the lack of experienced personnel. 
b. Clearly, one should avoid such situations by describing and maintaining the process properly. Still, unforeseen reasons to abort the treatment may always occur. The management should endorse the opt-out possibility in order to resist the mental pressure or temptation to use quick solutions or bypasses to push through the treatment in order to avoid annoyance about the situation by the patient and/or staff. Such ad-hoc solution seriously degrades the quality and safety of the treatment.

6. Risk management $[7,8,11,62,69,76,141,164]$

a. Risk analysis of the process chain reveals a lot about weak links in that chain.

b. Risk analysis should be performed concerning two aspects, each complementary to each other:

i. The risk for the quality and safety of the patient treatment;

ii. The risk for the organisation itself.

c. These risk analyses may yield conflicting outcomes: a high risk for the patient might be a low risk for the organisation and vice versa.

d. Coping with such conflicts, i.e. balancing between quality and safety versus efficiency and cost effectiveness, should be guided by the slogan for radiation protection:

"As low as reasonably achievable" (ALARA principle)

e. A multidisciplinary group is the best way to perform such risk analyses in every detail of the process chain.

f. Risk management always has to be a "loop process": it should be dynamic and evaluated periodically. The risk management should be part of a "plan-do-checkact (PDCA)" cycle. To perform risk analyses from accidents, near accidents and medical outcome, the institution should keep proper records of these issues. This is one source of information to start that PDCA-cycle. Another source is experience and reflection of the process.

\section{Statement 15 .}

Risk analyses should show potential risks in the patient treatment, workflow and department organisation, ranked according its performance, quality and safety damage to each aspect mentioned. Whereas procedures should be realized to limit those risks. But realize this: it is impossible to exclude all chances on (potential) errors. 


\subsection{The Process Control (Figure 2D)}

\subsubsection{Introduction}

A good quality assurance on the individual links of the clinical process chain does not guarantee a good overall quality of the patient treatment since checks on the individual parts of a chain seldom consider the mutual interactions between these parts. For example, the ICT data flow from one part of the chain to another. Or treatment related dataflow based on communication between persons and/or disciplines.

In summary:

- The overall uncertainty in the process chain, resulting in dose delivery to the patient, should be stated by an independent control method.

- It is the task of the department to ensure that the overall uncertainty is within the accuracy limits stated by the department.

- Those accuracy limits should be based on what is commonly accepted or supported by common experience.

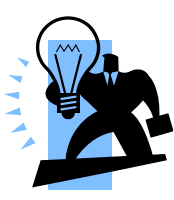

\section{Statement 16:}

Performing stereotactic treatment requires an end-to-end test on a regular basis, embedded in a well-implemented plan-do-check-act quality assurance cycle.

\subsubsection{General advice}

Setting up an independent end-to-end test is challenging since proper tools for this are not (always) available. Until they are, an independent verification should cover the largest possible parts of the whole process chain. Preferably, more than one verifications are carried out on overlapping parts of the process chain.

In this paragraph we present "general words of advice", staccato again, based on our experiences with setting up a good process control. Chapter 7 contains more detailed advice regarding the process control, specifically related to stereotactic equipment. 


\section{Start up.}

a) Make sure that the patient dose delivery is accurate, taking into account the (inter)national standards as setup by experts.

b) A risk analysis as discussed in the chapter "Process chain" is a premise before starting an end-to-end test. It identifies both technical and procedural weak links in the treatment chain.

c) With this knowledge, end-to-end tests can be setup with more attention, according to the PDCA-cycle.

d) When using a phantom, investigate whether or not quality control on that phantom is needed. For example, some phantoms can be decomposed to insert dose detectors like film or ionisation chamber, possibly changing its geometry.

\section{Overall Phantom Test: end-to-end test.}

a) An end-to-end test provides the best possible way to verify the overall quality of the complete stereotactic treatment process. It can be performed to check equipment and methodology on the one hand and to investigate the procedures, e.g. human factors and correct order of subsequent process steps, on the other hand. Both end-to-end tests are complementary and have to be applied to find and correct weak links in the process chain [e.g. 102, 124].

b) The main pitfall in setting up an end-to-end test is to be lost in details. To avoid this, it is important to keep the overall goal in perspective:

i) The target in a patient has to be irradiated correctly while organs at risk have to be spared.

ii) Check that the accuracy in absolute dose, dose distribution, the geometry of the patient and the target positioning are within the required limits.

iii) Find out the weak links in this process in quality, safety, efficiency and effectiveness, as discussed under "Process chain".

c) To avoid dangerous bias when analysing the results we advise to discuss the outcome of the end-to-end test with independent experts. Even then one has to be aware of possible bias.

d) Translate the end-to-end test into actual test-conditions:

i) Set up target and organs at risk in a phantom, perform the whole stereotactic treatment process on that phantom and detect in that phantom the absolute dose, 
dose distribution and position of that distribution with respect to target and organs at risk.

ii) Perform the institutes process chain, i.e. realise what is important to do and what not.

iii) Detect what accuracy is achieved with the end-to-end test and correlate it to the accuracy requested for the current process chain. Transfer that to the right materials to use. For example, use an appropriate detector.

iv) Translate that information into realistic improvements, according the plan-docheck-act cycle.

e) If a single end-to-end test cannot cover the whole chain, split it up into parts. To be sure over the whole chain, make overlaps between parts.

\section{Overall Phantom Test: class solution test}

a) With a class solution the process control and the "end-to-end" test can be performed more easily in the daily clinical routine, since individual patient data can be replaced by a standard patient dataset.

b) Rules to setup a class solution cannot be given. It depends on the way of working of the department. The quality of a class solution is, therefore, the responsibility of that department.

\section{Patient Specific Test: pre-treatment verification}

In the NCS report on IMRT QA [157], advice is given how to use pre-treatment verification. In our report we refer to those words of advice. Based on our experience, we would like to add the following considerations:

a) Class solutions, as discussed above, will not always provide the best individual patient treatment. In those situations pre-treatment verification is strongly advised to check the quality of the treatment plan, if possible.

b) Pre-treatment verification is strongly advised, both dosimetrical and geometrical, when starting with (new) stereotactic treatments.

c) Even for established class solutions it may be worthwhile to perform pre-treatment verification (once in a while) in order to verify the correspondence between computed and delivered dose, acknowledging possible inaccuracies of the MLC leaf positioning or uncertainties regarding data transport between treatment planning system and irradiation device. 
d) Pre-treatment verification is very valuable but one should realise, of course, that patient-specific uncertainties are ignored.

e) Pre-treatment verification helps to identify uncertainties in the whole treatment process. It is therefore advised to setup the pre-treatment verification as a systematic investigation process. Decide in advance what the objectives are, what number of patient plans should be included, how often should the treatment setup be verified, which type of detector and phantom should be used, what the action levels are and how the results are administered. In this way pre-treatment verification can help you to improve your "end-to-end" test and class solution.

\section{Patient Specific Test: in-vivo dosimetry}

In both the end-to-end test and the pre-treatment verification procedure, the patient influence on the dose delivery is excluded. This influence can only be determined using in-vivo dosimetry.

a) At this moment, in vivo dosimetry for stereotactic treatment is not developed. The first reason is that small fields do not allow to control simultaneously the positioning of the dose distribution with respect to patient anatomy. In-vivo dosimetry requires, therefore, the performance of a cone-beam CT during treatment (just before, during or just after). Second, the construction of the equipment used does not always allow in-vivo dosimetry, as in the case of the Gamma Knife.

b) In-vivo dosimetry is complementary to pre-treatment verification. Its advantage is that it records the actual dose delivered to the patient considering the actual patient anatomy. The main drawback is that it provides the answer after the treatment was completed, which might be too late due to the high-dose fractions if serious errors were to be found.

c) Concerning the end-to-end test, a systematic setup of in-vivo dosimetry yields additional information about the whole process chain, in particular regarding the influence of the patient. This cannot be investigated in the end-to end test.

\section{Follow up: medical analysis of patient treatments}

The whole stereotactic treatment process chain can be setup with a high technical quality, efficient and effective in the process. Still, its remains a shot in the dark if there is no adequate medical analysis about treatment response, local recurrence and complications. Outcome analysis can be a reason to optimise the process chain or the medical procedure. Although registration of outcome, recurrences and complications are 
well established quality parameters of good radiotherapy practice, we want to stress its value specifically for stereotactic treatments. 


\section{Conclusion}

Intracranial stereotactic treatment is a high-fraction dose, high-precision target positioning treatment technique that requires the highest level of accuracy and safety in patient treatments, as errors in treatment often result in irreparable and severe damage to the patient. Therefore, the implementation, maintenance and improvement of this type of techniques require a high awareness by management and professional staff that actions are necessary in different areas. For the stereotactic treatment as described in this report, the words of advice given can be summarized to what has been presented in the systematic review.

Statement, Prerequisites 17.

Question: What do we need to perform intracranial stereotactic treatment?

Quote: Embarking stereotactic treatment requires dedication from the whole organisation.

Statement, Equipment and Methodology 18.

Question: What about your equipment and methods to be implemented?

Quote: Preferably, stereotactic treatment is performed with "single manufacturer" devices.

Statement, Process chain 19.

Question: What about the quality and safety level of your process system?

Quote: The process should be setup straightforward and has well-defined "stop" control points. 
Statement, Process control 20.

Question: What about your overall treatment quality control?

Quote: Stereotactic treatment requires end-to-end tests and well-trained and experienced staff.

Statement, this report 21 .

The advice given in this report is based on present-day experiences and should not be considered to be complete. It is, therefore, very important that each institution performing stereotactic treatment keeps up-to-date with the progress in the field and anticipates on that. Only sharing information can yield a real improvement in patient cure and care. 


\section{Appendix: Equipment and Methodology / Process chain}

This Appendix provides detailed information about the subject "Methodology and Equipment" (Figure 2B; table: middle column) and "Process chain" (Figure 2C; table: right column). Those details are combined in a single appendix as most of them are related.

\begin{tabular}{|c|c|c|c|}
\hline & \multicolumn{3}{|c|}{ Fixation: Frame } \\
\hline Aspect & & Methodology and Equipment (Link to Figure 2B) & Process chain (Link to Figure 2C) \\
\hline Safe use & 1) & $\begin{array}{l}\text { Orthogonality of the stereotactic frames and its correct alignment to } \\
\text { coordinate's reference frame must be thoroughly investigated before first } \\
\text { use and subsequently during QC. } \\
\text { If an independent test is not possible, than orthogonality should be } \\
\text { verified by image fusion of two imaging modalities, e.g. MRI and CT } \\
\text { images. } \\
\text { Treatment planning on MRI images only requires an approved MRI } \\
\text { compatible localizer frame. } \\
\text { Target positioning box: check orthogonality (see 1) and integrity. Verify } \\
\text { before first use the consistency of the marks on the localizer box and the } \\
\text { marks on the plots generated by the TPS. } \\
\text { Delineation of an AVM (Arteriovenous malformation) is often supported by } \\
\text { two orthogonal X-ray angiograms. This requires a special X-ray angio- } \\
\text { localizer frame. }\end{array}$ & $\begin{array}{l}\text { 1) Placement of a stereotactic frame (by } \\
\text { neurosurgeon) requires careful } \\
\text { considerations. Participation of radiation } \\
\text { oncologist or medical physicist is } \\
\text { recommended. Incorrect placement may lead } \\
\text { to patient discomfort, cause unacceptable } \\
\text { scatter in images, hiding landmarks for IGRT } \\
\text { (e.g. ExacTrac system), restrict FOV during } \\
\text { imaging, obstruct mounting accessories, } \\
\text { etc., and may ultimately lead to re-placement } \\
\text { of the frame. }\end{array}$ \\
\hline
\end{tabular}




\begin{tabular}{|c|c|c|}
\hline & \multicolumn{2}{|c|}{ Fixation: Image guided Immobilisation } \\
\hline Aspect & Methodology and Equipment (Link to Figure 2B) & Process chain (Link to Figure $2 \mathrm{C}$ ) \\
\hline Use & $\begin{array}{l}\text { 1) Frames for Image Guided RT (IGRT) are mostly dedicated to a particular } \\
\text { IGRT system. It is mandatory to follow the manufacture guidelines. } \\
\text { 2) Before first use the intra- and/or inter fraction accuracy must be assessed. } \\
\text { 3) For each (new) class of clinical application one should compare the } \\
\text { required accuracy with specified and/or determined accuracy. Acceptance } \\
\text { test of the manufacture do not necessarily cover all consumer's intended } \\
\text { clinical applications. }\end{array}$ & \\
\hline
\end{tabular}

\begin{tabular}{|c|c|c|c|}
\hline & \multicolumn{3}{|c|}{ Imaging (for Treatment Planning): General } \\
\hline Aspect & & Methodology and Equipment (Link to Figure 2B) & Process chain (Link to Figure 2C) \\
\hline Use & 1) & $\begin{array}{l}\text { Data transport from imaging modality to Treatment } \\
\text { Planning System has to be straightforward. } \\
\text { Imaging modalities have to be DICOM and } \\
\text { preferably IHE-compliant. } \\
\text { The image quality has to be appropriate, e.g.: } \\
\text { a) Resolution suited for construction of DRR. } \\
\text { b) Geometric distortion of MR images should be } \\
\text { as low as possible. }\end{array}$ & $\begin{array}{l}\text { Specific for combination of CT with Linac: } \\
\text { 1) Caution with the horizontal alignment on the CT when pitch and roll } \\
\text { setup errors cannot be corrected on the Linac. } \\
\text { 2) Be aware of geometric aberrations / distortions due to patient } \\
\text { motion, especially between non-coupled PET and CT: a DICOM-link } \\
\text { only assures that PET and CT are from the same series. }\end{array}$ \\
\hline
\end{tabular}




\begin{tabular}{|c|c|c|}
\hline & \multicolumn{2}{|c|}{ Imaging (for Treatment Planning): CT } \\
\hline Aspect & Methodology and Equipment (Link to Figure 2B) & Process chain (Link to Figure $2 \mathrm{C}$ ) \\
\hline Use & $\begin{array}{l}\text { 3D-CT: } \\
\text { 1) Geometrical accuracy of the 3D reconstruction } \\
\text { must be validated using phantoms or a localiser } \\
\text { box with exact determined geometrical markers. } \\
\text { 2) Deviations between expected and actual positions } \\
\text { must be smaller than the pixel size of the CT } \\
\text { image (<1 mm) } \\
\text { 4D-CT: } \\
\text { 3) For intracranial stereotactic treatment 4D-CT is not } \\
\text { applicable as imaging tool. } \\
\text { 4) Although out of scope of this protocol: for "extra- } \\
\text { cranial stereotactic treatment" gathering, analysis } \\
\text { and transport of this data should be a well- } \\
\text { controlled imaging tool. }\end{array}$ & $\begin{array}{l}\text { Linac and Cyberknife specific: } \\
\text { 1) Be aware of the effective bore opening of the scanner: avoid } \\
\text { collisions between patient and CT-scanner. } \\
\text { 2) Choose scan resolution: it influences the DRR-quality for target } \\
\text { positioning. } \\
\text { 3) Select the appropriate field of view for 3D CT, considering: } \\
\text { a) Delineation of target and organs at risk; } \\
\text { b) Treatment setup, i.e. non-coplanar dose calculation should be } \\
\text { possible. } \\
\text { c) Patient positioning verification possibility during treatment (e.g } \\
\text { 4D-CT: } \\
\text { 4) Although out of scope of this protocol: motion registration of the } \\
\text { target / organs at risk should be compatible with such systems on } \\
\text { Linac or Cyberknife if target tracking is used as irradiation } \\
\text { technique. }\end{array}$ \\
\hline
\end{tabular}




\section{Imaging (for Treatment Planning): Angio}

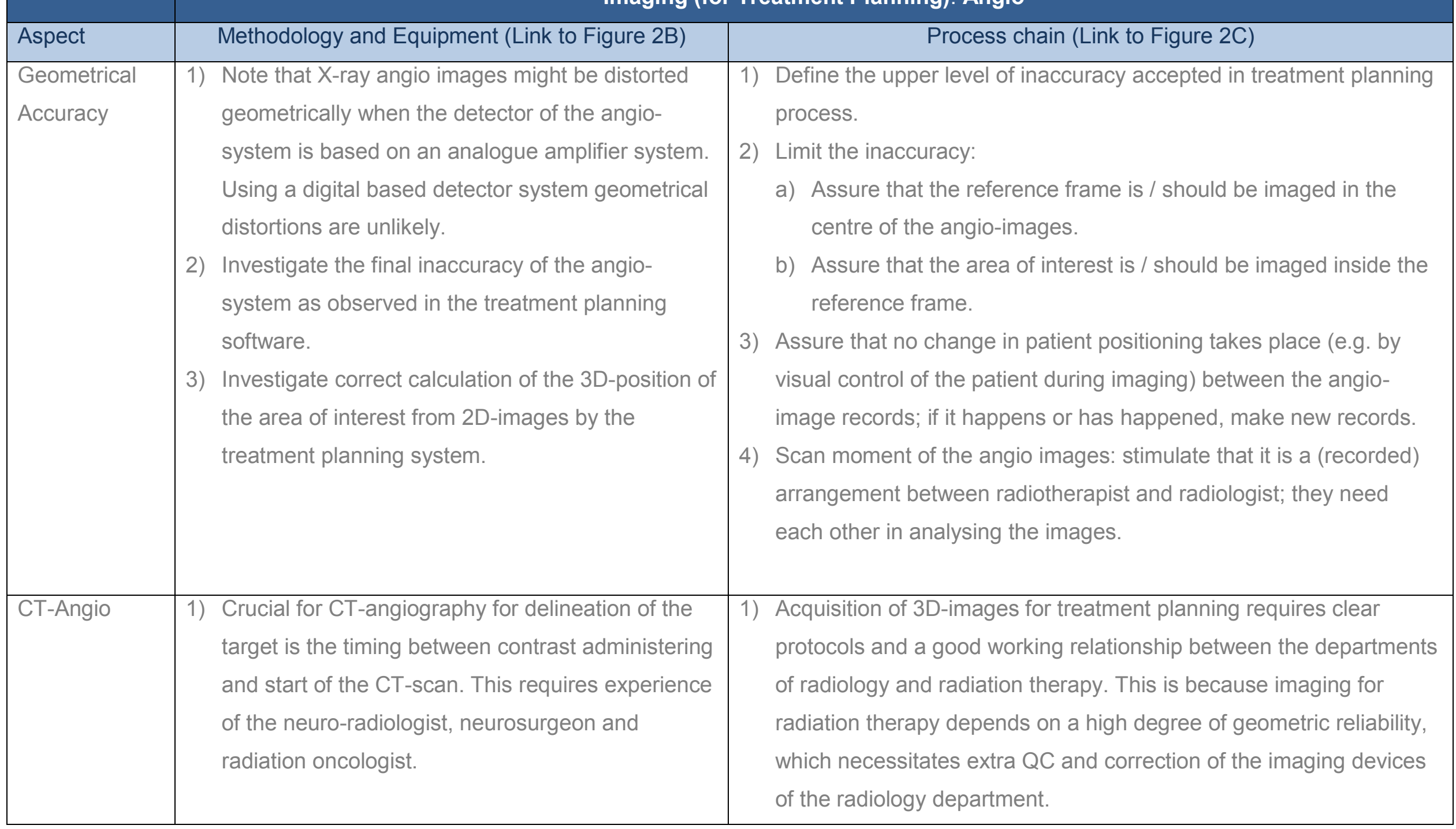




\begin{tabular}{|c|c|c|}
\hline & \multicolumn{2}{|c|}{ Imaging (for Treatment Planning): Angio } \\
\hline Aspect & Methodology and Equipment (Link to Figure 2B) & Process chain (Link to Figure $2 \mathrm{C}$ ) \\
\hline & & $\begin{array}{l}\text { 2) Two types of 3D angiography can be distinguished: } \\
\text { a) CT-angio using a standard CT scanner with contrast injection. } \\
\text { Image acquisition is performed after a patient-specific delay, } \\
\text { based on carotid artery contrast tracking. This can be combined } \\
\text { with } 2 \mathrm{D} \text { angiography, desirable in the case of Cyberknife. } \\
\text { b) Cone Beam CT (CB-CT) with digital subtraction angiography } \\
\text { (DSA). } \\
\text { 3) Final datasets of both modalities mentioned above may not be } \\
\text { compliant with stringent Dicom input format of the TPS. One } \\
\text { solution is to develop a work around using in-house software. } \\
\text { However, a work around is not recommended in this report. }\end{array}$ \\
\hline MRI-Angio & 1) References only [e.g. 28] & $\begin{array}{l}\text { 1) Keep in mind that MRI-Angio images might not be Dicom-connected } \\
\text { (registered) to other MRI-series. } \\
\text { 2) This mean that registration of MRI-Angio images with other MRI- } \\
\text { images should be performed carefully. } \\
\text { 3) During the report production process, limited expertise was present } \\
\text { and hardly any literature available. So keep up with recent literature. }\end{array}$ \\
\hline
\end{tabular}




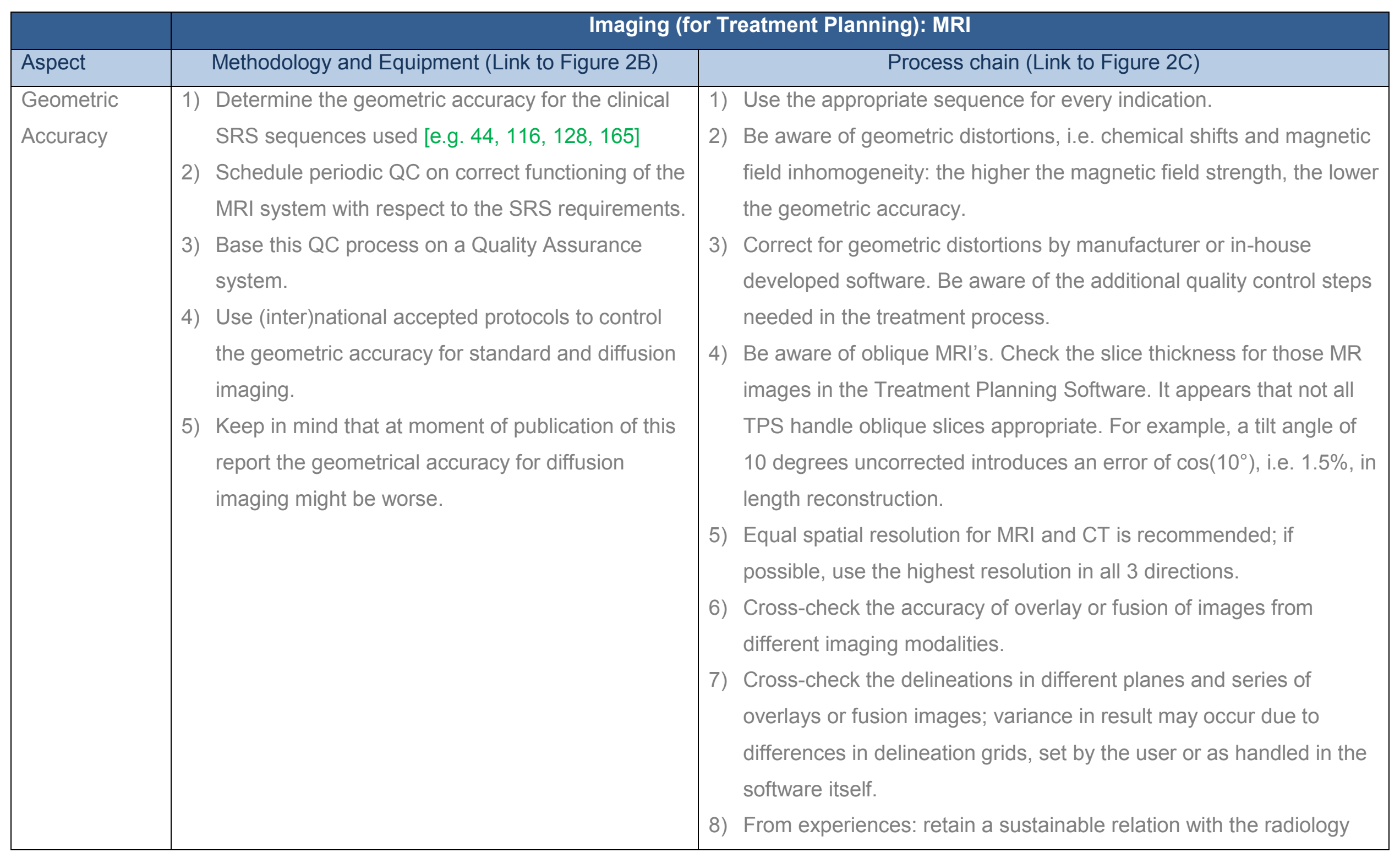




\begin{tabular}{|l|l|l|}
\hline \multirow{2}{*}{ Aspect } & \multicolumn{2}{|c|}{ Imaging (for Treatment Planning): MRI } \\
\hline & & \multicolumn{1}{c|}{ Process chain (Link to Figure 2C) } \\
\cline { 2 - 4 } & & $\begin{array}{l}\text { and the nuclear medicine department if MRI- and PET(-CT) images } \\
\text { are obtained from those departments. For those departments it is } \\
\text { not always obvious what the required quality and geometrical } \\
\text { accuracy of the images should be for radiotherapy treatment. }\end{array}$ \\
& & \\
& &
\end{tabular}

\begin{tabular}{|c|c|c|}
\hline & \multicolumn{2}{|c|}{ Imaging (for Treatment Planning): PET (-CT) } \\
\hline Aspect & Methodology and Equipment (Link to Figure 2B) & Process chain (Link to Figure 2C) \\
\hline Use & $\begin{array}{l}\text { 1) Only use PET in combination with a CT from the } \\
\text { same imaging series, i.e. yielding a DICOM- } \\
\text { coupled PET-CT. }\end{array}$ & $\begin{array}{l}\text { 1) Do not use PET for positioning or as primary delineation modality as } \\
\text { spatial resolution and delineation criteria, i.e. specific uptake values, } \\
\text { are too small (specific) for high accuracy needed in intracranial } \\
\text { stereotactic treatment. } \\
\text { 2) So: PET is an appropriate tool only to determine staging and draw } \\
\text { attention to relevant regions on CT and MRI. }\end{array}$ \\
\hline
\end{tabular}




\begin{tabular}{|c|c|c|}
\hline & \multicolumn{2}{|c|}{ Treatment Planning: Image Handling } \\
\hline Aspect & Methodology and Equipment (Link to Figure 2B) & Process chain (Link to Figure $2 \mathrm{C}$ ) \\
\hline $\begin{array}{l}\text { Co- } \\
\text { Registration }\end{array}$ & $\begin{array}{l}\text { 1) It should be possible in the TPS to create an } \\
\text { overlay-image set. } \\
\text { 2) On the overlay-image set at least co- } \\
\text { registration is possible; } \\
\text { 3) This co-registration must be at least based on } \\
\text { 3D total scanned volumes. } \\
\text { 4) In intra-cranial stereotactic treatment } \\
\text { deformable registration is not applicable. } \\
\text { 5) However, if used, such algorithms have to be } \\
\text { understood very well. } \\
\text { 6) An independent check of the method and the } \\
\text { accuracy of the co-registration should be } \\
\text { performed. } \\
\text { 7) This co-registration should be recorded within } \\
\text { the treatment planning process so that it } \\
\text { remains available for post-evaluation. }\end{array}$ & $\begin{array}{l}\text { 1) Basic of all is the selection of the reference image set }(\mathrm{CT}, \mathrm{MRI}, \ldots) \text { which } \\
\text { will be used for dose calculation. } \\
\text { 2) This reference image set is often handled in software as the "master- } \\
\text { image set": to this image set the dose distribution and treatment time is } \\
\text { calculated } \\
\text { 3) Using automatic registration tools: } \\
\text { a) Do not completely rely on automatic registration! Verify the outcome! } \\
\text { b) An independent verification tool or method at least over several } \\
\text { slices should be implemented. } \\
\text { 4) Be aware that each image dataset can be oriented differently in space, } \\
\text { e.g. rotated relative to each other. This can induce inaccuracies in the } \\
\text { co-registration process. } \\
\text { 5) Imaging sets should be representable for: } \\
\text { a) The actual anatomy during treatment delivery; } \\
\text { b) The treatment position as close as possible (e.g. MRI images might } \\
\text { be produced in a slightly different outlining of the patient with respect } \\
\text { to the desired treatment position) }\end{array}$ \\
\hline $\begin{array}{l}\text { Fused-image } \\
\text { set }\end{array}$ & $\begin{array}{l}\text { 1) Image fusion: is allowed in treatment planning } \\
\text { [54]. } \\
\text { 2) Similar aspects as related to co-registration }\end{array}$ & $\begin{array}{l}\text { 1) Similar aspects as related to co-registration have to considered. } \\
\text { 2) The "master-image set" becomes the fused-image set. Be aware of the } \\
\text { possible resetting of } \mathrm{HU} \text { values. }\end{array}$ \\
\hline
\end{tabular}




\begin{tabular}{|c|c|c|}
\hline & \multicolumn{2}{|c|}{ Treatment Planning: Image Handling } \\
\hline Aspect & Methodology and Equipment (Link to Figure 2B) & Process chain (Link to Figure 2C) \\
\hline & $\begin{array}{l}\text { have to be considered. } \\
\text { 3) In clinical use a fused-image set is named too } \\
\text { as "integrated image set" }\end{array}$ & \\
\hline Fiducial & $\begin{array}{l}\text { 1) If fiducials will be used in patient and beam } \\
\text { data transport, the TPS software should be } \\
\text { tested in a similar way as described for co- } \\
\text { registration. }\end{array}$ & $\begin{array}{l}\text { 1) Data transport based on frame information can be replaced only by } \\
\text { internal structures or markers as long as those structures or markers } \\
\text { can be handled as fiducials. }\end{array}$ \\
\hline
\end{tabular}


Treatment Planning: Dosimetry and Beam Data

\begin{tabular}{|c|c|c|}
\hline Aspect & Methodology and Equipment (Link to Figure 2B) & Process chain (Figure 2C) \\
\hline Dosimetry & $\begin{array}{l}\text { 1) The ability to accurately deliver the intended dose and ensuring that this desired dose } \\
\text { distribution coincides with the desired point inside the patient is critical in stereotactic } \\
\text { treatment. } \\
\text { 2) However, absolute and relative small field dose measurement needed for this is not easy } \\
\text { to do; the field is under investigation. Resources related to means and manpower should } \\
\text { be available. } \\
\text { 3) Measuring correct beam characteristics for stereotactic treatment yields two pitfalls, } \\
\text { related to: } \\
\text { a) The dosimetry system used. } \\
\text { b) The controllability of the data. } \\
\text { 4) In Chapter } 8 \text { \& } 9 \text { several aspects to be considered have been discussed shortly. } \\
\text { 5) Starting reference [17, } 18,33,50,150,152]\end{array}$ & \\
\hline
\end{tabular}


Treatment Planning: Dose Calculation

\begin{tabular}{|c|c|c|}
\hline Aspect & Methodology and Equipment (Link to Figure 2B) & Process chain (Figure 2C) \\
\hline Acceptance & $\begin{array}{l}\text { 1) The treatment planning system should be commissioned and tested as advised in } \\
\text { (inter)national standards, taking into account the different ways dose calculation can be } \\
\text { performed, i.e. forward and inverse planning like IMRT and VMAT / RapidArc [e.g. 27; } \\
\text { 157] } \\
\text { 2) Caveat: information will never be "overall complete", i.e. given information should not } \\
\text { interpreted as "complete enough to stop testing". } \\
\text { 3) Information about the system provide by the manufacturer might be insufficient with } \\
\text { respect to tests to be performed. } \\
\text { 4) Don't test the treatment planning system as system only. It should be tested too in the } \\
\text { clinical treatment process, as the planning system is a crucial device in the data transport } \\
\text { of patient information and generation of treatment parameters. For example: MLC-field } \\
\text { shape and orientation, jaw setting with respect to MLC and cones. } \\
\text { 5) The treatment planning system should be tuned for small-field dose calculation. } \\
\text { a) If a compromise in accuracy required for large - and small-field dose calculation is not } \\
\text { possible, it might be helpful to implement } 2 \text { treatment units in the planning system (for } \\
\text { stereotactic fields and for standard fields).In that case the dedicated stereotactic } \\
\text { machine is often restricted to limited field sizes. } \\
\text { b) For Linacs this might be a "requirement" for treatment applying cylindrical cones: } \\
\text { restricted or / and fixed field sizes are recommended. } \\
\text { c) Accuracy levels to be fulfilled are not easily to given. E.g. Chapter } 10 \text {. } \\
\text { d) However, they should be related to nowadays standards about stereotactic treatment. }\end{array}$ & \\
\hline
\end{tabular}




\begin{tabular}{|c|c|c|c|}
\hline & \multicolumn{3}{|c|}{ Treatment Planning: Dose Calculation } \\
\hline \multirow[t]{2}{*}{ Aspect } & & Methodology and Equipment (Link to Figure 2B) & Process chain (Figure 2C) \\
\hline & & $\begin{array}{l}\text { e) Therefore, each institution performing stereotactic treatment should define their } \\
\text { accuracy levels used in the clinical process; it helps to improve the accuracy and } \\
\text { safety of the treatment (inter) nationally. } \\
\text { In Chapter } 10 \text { more information is given about control on commissioned data. }\end{array}$ & \\
\hline Frames & $\begin{array}{l}\text { 1) } \\
\text { 2) } \\
\text { 3) } \\
\text { 4) }\end{array}$ & $\begin{array}{l}\text { The treatment planning system has the possibility to handle the frames used in the } \\
\text { clinical process of the department. } \\
\text { This "frame handling" should be unambiguous, correct and highly-accurate in its transfer } \\
\text { from imaged patient information, i.e. target and critical organs, to positioning of the target } \\
\text { and critical organs with respect to the radiation sources, i.e. the }{ }^{60} \text { Co of the Gamma Knife } \\
\text { and the isocentre of the Linac and Cyberknife. } \\
\text { It is advised to test this extensively, for instance using a phantom. } \\
\text { Point } 1 \text { and } 2 \text { above are not evident for frames not provided by the Treatment Planning } \\
\text { System manufacturer. }\end{array}$ & \\
\hline
\end{tabular}




\begin{tabular}{|c|c|c|}
\hline & Treat & ent Planning: Localisation \& Delineation \\
\hline Aspect & Methodology and Equipment (Figure 2B) & Process chain (Link to Figure $2 \mathrm{C}$ ) \\
\hline Delineation & See NCS 15 [27] & 1. See NCS 15 [27] \\
\hline Localization & & $\begin{array}{l}\text { 1) Localization on different image modalities: } \\
\text { a) Define the reference image modality for the target and organs at risk. } \\
\text { b) Limits should be set for the accepted differences between the image } \\
\text { modalities. Those differences are dependent on slice thickness, } \\
\text { orientation differences, pixel size,...... } \\
\text { 2) If no such limits can be provided, we strongly recommend to work only with } \\
\text { experienced staff that know knowing the pitfalls of the registration process. }\end{array}$ \\
\hline
\end{tabular}




\begin{tabular}{|c|c|c|}
\hline \multicolumn{3}{|c|}{ Treatment Planning: Dose prescription and Treatment Planning } \\
\hline Aspect & Methodology and Equipment (Figure 2B) & Process chain (Link to Figure $2 \mathrm{C}$ ) \\
\hline $\begin{array}{l}\text { Basic planning } \\
\text { items }\end{array}$ & & $\begin{array}{l}\text { 1) Close nearness of a structure at risk to the target is the main issue in intra- } \\
\text { cranial stereotactic treatment. } \\
\text { 2) Therefore, it is chosen in stereotactic treatment to create dose distributions } \\
\text { around the planning target volume with the strongest dose gradient possible to } \\
\text { outside regions. For instance to the } 20 \% \text { dose level. } \\
\text { 3) The side effect is a large dose inhomogeneity over the target volume itself. } \\
\text { 4) In Linac and Cyberknife based intracranial stereotactic treatment it is standard } \\
\text { to prescribe the dose "at the } 80 \% \text { " isodose around the planning target volume. } \\
\text { 5) For Gamma Knife dose prescription at variable levels might be used. } \\
\text { 6) However, other dose prescriptions can be chosen for, e.g. using the ICRU } \\
\text { criteria [3, } 4,5] \\
\text { 7) Irrespectively of the choice made, be transparent about the dose prescription } \\
\text { applied to the planning target volume. } \\
\text { 8) Dose coverage target volume. } \\
\text { a) Even in intracranial stereotactic treatment it is desired to obtain a coverage } \\
\text { index equal to unity, as long as no conflict yields with dose values } \\
\text { acceptable for structures at risk. } \\
\text { b) The value of conformity index, heterogeneity index and homogeneity index } \\
\text { is of (much) less importance in intra-cranial stereotactic treatment. } \\
\text { 9) Electron equilibrium. } \\
\text { a) Realize that the lack of electron equilibrium is much more dominant for field }\end{array}$ \\
\hline
\end{tabular}




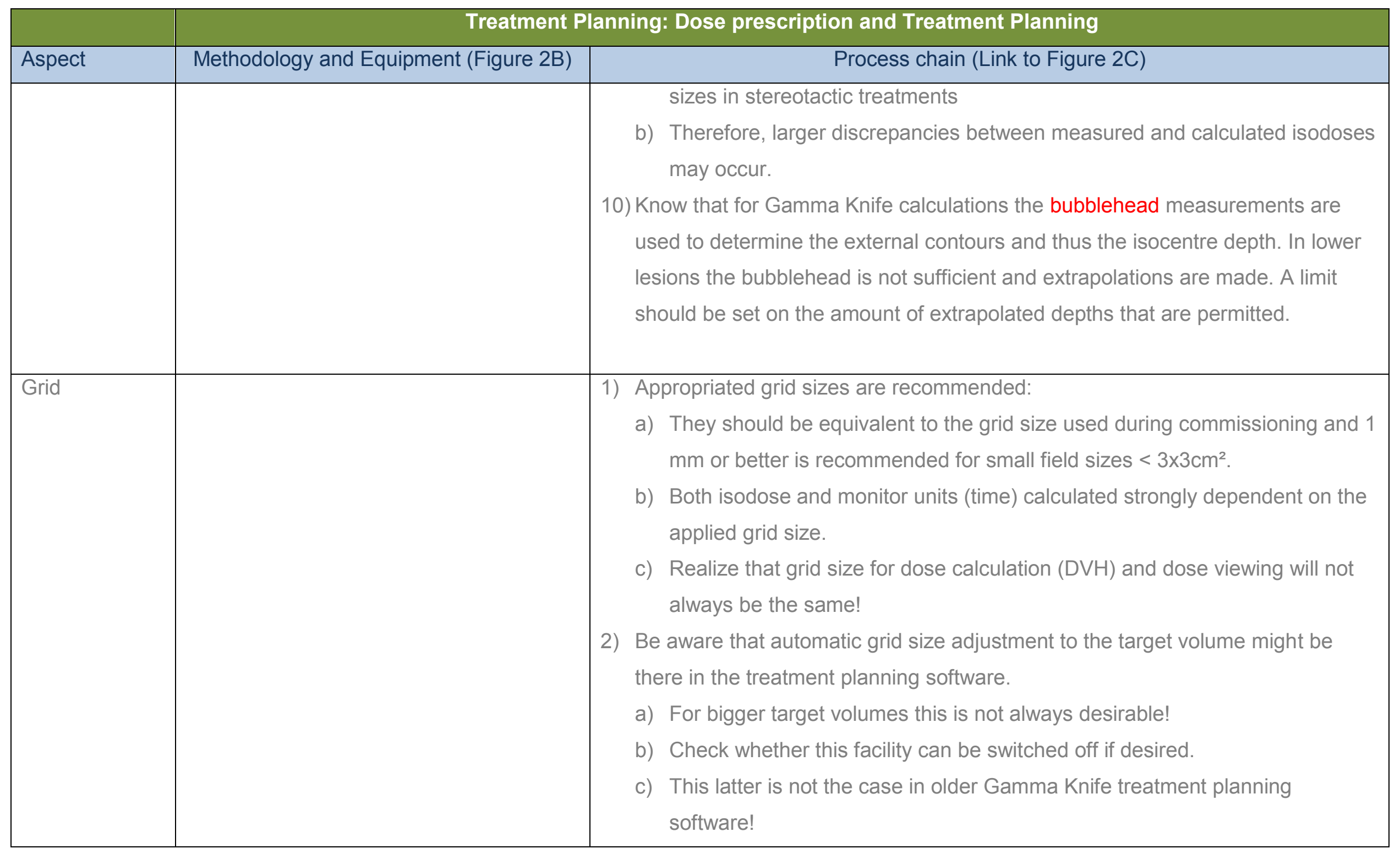




\begin{tabular}{|c|c|c|}
\hline \multicolumn{3}{|c|}{ Treatment Planning: Dose prescription and Treatment Planning } \\
\hline Aspect & Methodology and Equipment (Figure 2B) & Process chain (Link to Figure $2 \mathrm{C}$ ) \\
\hline Margins & & $\begin{array}{l}\text { 1) In combination with the standard stereotactic dose prescription (see above) a } 0 \\
\text { or } 1 \mathrm{~mm} \text { margin is used in general, to limit the dose to critical organs. } \\
\text { 2) The use of a larger margin, based on fixation and positioning accuracy } \\
\text { achievable, has to be weighed against clinical outcome. } \\
\text { 3) The medical concept behind limiting the margins is based on the idea that even } \\
\text { in the steep dose gradient region, e.g. for the Linac en Cyberknife the } 80-20 \% \\
\text { region, the fraction dose is high enough to sterilize that region. } \\
\text { 4) Keep in mind: using the ICRU criteria, it might yield a higher dose to critical } \\
\text { structures due to a larger dose fall-off volume from } 95 \% \text { to } 20 \% \text { isodose. } \\
\text { 5) Irrespective of what choice is made, be transparent, internally and externally, } \\
\text { about the margin between planning and clinical target volume. }\end{array}$ \\
\hline $\begin{array}{l}\text { Multiple } \\
\text { isocentres }\end{array}$ & & $\begin{array}{l}\text { 1) Use isocentre names (Gamma Knife: i.e. matrix names) referring to the } \\
\text { corresponding target site, to improve safety on the treatment unit. } \\
\text { 2) If multiple isocentres are treated, calculate and evaluate a "sum plan" too, as } \\
\text { peripheral dose from one treatment plan for isocentre X might add to the dose } \\
\text { distribution of isocentre Y (e.g. Gamma Knife and Eclipse) } \\
\text { 3) Dose calculation matrix: be aware different grid size can be used regarding the } \\
\text { treatment isocentre. } \\
\text { 4) Be aware of the accuracy of peripheral dose calculations in Gamma Knife }\end{array}$ \\
\hline
\end{tabular}




\begin{tabular}{|c|c|c|}
\hline \multirow[b]{2}{*}{ Aspect } & \multicolumn{2}{|c|}{ Treatment Planning: Dose prescription and Treatment Planning } \\
\hline & Methodology and Equipment (Figure 2B) & Process chain (Link to Figure 2C) \\
\hline & & $\begin{array}{l}\text { planning. Dose outside the matrix is not calculated with the same grid size and } \\
\text { is, therefore, less accurate. }\end{array}$ \\
\hline Couch & & $\begin{array}{l}\text { 1) While using a rigid reference frame for each treatment isocentre, table values } \\
\text { (length, width and height) can be calculated and used for position verification. } \\
\text { 2) Realize that a dedicated tolerance table, e.g. with narrow positioning window, } \\
\text { has to be applied for this verification, based on a careful evaluation of the } \\
\text { accuracy achievable. } \\
\text { Linac \& Gamma Knife: } \\
\text { 3) While using stereotactic radiotherapy a capture of the couch parameters after } \\
\text { verification is a very useful tool for treatment positioning during positioning of } \\
\text { the patient in the remaining fractions. }\end{array}$ \\
\hline $\begin{array}{l}\text { Final points } \\
\text { about planning }\end{array}$ & & $\begin{array}{l}\text { 1) Verification-fields: if possible incorporate verification fields for target-isocentre } \\
\text { verification. } \\
\text { 2) "Small-field" calculation: independent MU verification for small fields is } \\
\text { recommended [e.g. 100]. } \\
\text { 3) Final plan: use a printed / digital document for supervision of the Record \& } \\
\text { Verify system during treatment delivery. } \\
\text { 4) Linac, back-up jaws: }\end{array}$ \\
\hline
\end{tabular}


Treatment Planning: Dose prescription and Treatment Planning

\begin{tabular}{|l|l|l|l}
\hline Aspect & Methodology and Equipment (Figure 2B) & Process chain (Link to Figure 2C)
\end{tabular}

a) Use a small margin (max. $5 \mathrm{~mm}$ ) between largest leaf setting and back-up jaw position

b) An automatic backup jaw position is recommended (remember the New York Evanston irradiation incident ${ }^{6}$ ) 
Treatment Planning: Planning Control

\begin{tabular}{|c|c|c|}
\hline \multicolumn{3}{|c|}{ Treatment Planning: Planning Control } \\
\hline Aspect & Methodology and Equipment (Link to Figure 2B) & Process chain (Link to Figure $2 \mathrm{C}$ ) \\
\hline Limits & $\begin{array}{l}\text { 1) Note the limits in the dose calculation with } \\
\text { respect to the limits on the treatment device. } \\
\text { For example: angles on which collisions will } \\
\text { occur, number of monitor units per arc, } \\
\text { maximum field size, maximum and minimum } \\
\text { MU per degree etc. }\end{array}$ & $\begin{array}{l}\text { 1) Control of the planning outcome; system and method. } \\
\text { a) A check of the dose distribution should be performed by an } \\
\text { independent observer, not being "the planner". } \\
\text { b) In this check the use of a short-list of control issues is recommended. } \\
\text { c) Standard treatment plan verification protocol can be used as a referral } \\
\text { of parts of the verification process. } \\
\text { 2) Monitor Units or treatment time (Gamma Knife) should be checked. } \\
\text { a) Guideline, based on experience, might be that for an arbitrary field } \\
\text { difference between MU calculated by the treatment planning system } \\
\text { and the control program can reach } 5 \% \text { for field sizes < } 1 \mathrm{~cm}^{2} \text { [102] } \\
\text { b) But even for such small field sizes the average MU calculated in both } \\
\text { procedures is often within } 3 \% \text {. } \\
\text { c) Data transfer from planning system to record \& verify (R\&V) system } \\
\text { should be checked carefully; probably MU / treatment time present in } \\
\text { the R\&V system can be the base of the check mentioned above. } \\
\text { 3) Example of the planning control process might be: } \\
\text { a) RTT's : perform the treatment planning; } \\
\text { b) Physics: redaction of the treatment planning process and participation } \\
\text { of the planning control. } \\
\text { 4) Compare exact position of the centre gravity of each target volume in the } \\
\text { stereotactic space using some manual verification. }\end{array}$ \\
\hline
\end{tabular}


Treatment Planning: Planning Control

\begin{tabular}{|c|c|c|}
\hline Aspect & Methodology and Equipment (Link to Figure 2B) & Process chain (Link to Figure 2C) \\
\hline & & $\begin{array}{l}\text { 5) If verification of the cone or aperture diameter is not included in the record } \\
\text { and verification system, implement an independent verification method in } \\
\text { the treatment protocol } \\
\text { 6) Collision test between treatment device and patient: } \\
\text { a) Those test should be a part of the planning control process } \\
\text { b) If a collision is observed plan adaptation should be performed; no } \\
\text { adaption during treatment is recommended: don't bypass process } \\
\text { checkpoints! }\end{array}$ \\
\hline
\end{tabular}




\begin{tabular}{|c|c|c|}
\hline & \multicolumn{2}{|c|}{ Treatment Positioning: General } \\
\hline Aspect & Methodology and Equipment (Link to Figure 2B) & Process chain (Link to Figure $2 \mathrm{C}$ ) \\
\hline Checks & $\begin{array}{l}\text { 1) Investigate and test all (chain) aspects about patient } \\
\text { positioning: what is possible and what not. } \\
\text { 2) The help of the manufacturers can be helpful, especially } \\
\text { when devices of different manufacturers are involved. } \\
\text { 3) Investigate which quality checks can be performed on a } \\
\text { regular basis, i.e. during regular quality control time instead } \\
\text { of patient care time; the latter is "expensive" time. } \\
\text { 4) Investigate the check-up procedure which should be } \\
\text { followed during patient treatment. }\end{array}$ & $\begin{array}{l}\text { Collisions: } \\
\text { 1) Be aware off and so careful about probable collisions } \\
\text { between patient and (parts of) the treatment device. } \\
\text { Reason is that in most cases a wide range of treatment } \\
\text { head and couch position is used increasing highly the } \\
\text { chance on collisions. } \\
\text { 2) Be aware that most collision detection devices or software } \\
\text { available on the treatment machine do not predict all } \\
\text { probable collision situations. } \\
\text { 3) Be aware that it is cumbersome or even not possible to } \\
\text { correct on-line patient treatment if a collision occurs during } \\
\text { treatment. If so, restart the process from "Treatment } \\
\text { planning". } \\
\text { 4) Experience: a dummy run just before treatment might be } \\
\text { helpful to protect collisions. } \\
\text { Collisions, Cyberknife typically. } \\
\text { 5) Just make the correct choice for the robotic arm path way. } \\
\text { Note that there is a "body" and "head" path way. If the } \\
\text { target location is connected to the wrong path way, a } \\
\text { collision might be expected! }\end{array}$ \\
\hline
\end{tabular}




\begin{tabular}{|c|c|c|}
\hline & & $\begin{array}{l}\text { 6) Just note the problem with stretched patient arms. } \\
\text { 7) Just note the problem with an isocentre positioned near to } \\
\text { the couch, i.e. irradiating the patient from below the couch. } \\
\text { Collisions, Gamma Knife typically. } \\
\text { 8) Note that some gamma angle positions increase the } \\
\text { chance on a collision depending on the target position. } \\
\text { Therefore, a dummy run should always be done. }\end{array}$ \\
\hline Accuracy & $\begin{array}{l}\text { 1) The overall accuracy in positioning of the target to the } \\
\text { isocentre should be within } 1 \mathrm{~mm} \text {, irrespective the fixation } \\
\text { and positioning technique used. } \\
\text { 2) In clinical routine the error between the actual dose } \\
\text { distribution position and the intended position with respect } \\
\text { to the target is often named "targeting error". }\end{array}$ & \\
\hline Safety & $\begin{array}{l}\text { 1) Train people to unfasten patients from the treatment couch } \\
\text { effectively and efficient: in fixed position an unexpected } \\
\text { physical response of the patient as e.g. coughing or an } \\
\text { epileptic attack can be very dangerous for the patient. }\end{array}$ & $\begin{array}{l}\text { 1) Positioning of patient and devices, e.g. add-ons, should be } \\
\text { checked by an independent qualified person. }\end{array}$ \\
\hline
\end{tabular}




\begin{tabular}{|c|c|c|}
\hline & \multicolumn{2}{|c|}{ Treatment Positioning: Mechanical systems } \\
\hline Aspect & Methodology and Equipment (Link to Figure 2B) & Process chain (Link to Figure $2 \mathrm{C}$ ) \\
\hline Quality Control & $\begin{array}{l}\text { Lasers (Cyberknife; Linac) } \\
\text { 1) The laser system should be aligned as an orthogonal } \\
\text { system, within an accuracy of } 1 \text { mrad }^{7} \text { at maximum. } \\
\text { 2) Alignment of the laser system at the (mechanical) isocentre } \\
\text { should be performed with the Winston Lutz test. } \\
\text { 3) The accuracy of this alignment should be better than } 0.25 \\
\text { mm, unless on-line target position verification and } \\
\text { correction guarantee target positioning within accuracy } \\
\text { limits stated by the institution. See statement } 11 . \\
\text { 4) The short and long term stability should be investigated } \\
\text { and taken into account in the regular quality control, or } \\
\text { even in a pre-treatment verification procedure. } \\
\text { 5) If a pre-treatment verification procedure of the laser system } \\
\text { is required, a quick effective and efficient scan procedure, } \\
\text { e.g. } 5 \text { minutes at most, should be developed. Verification } \\
\text { during "patient time" is "expensive time". } \\
\text { Immobilisation \& Lasers } \\
\text { 6) For invasive frame based treatments the laser system is }\end{array}$ & $\begin{array}{l}\text { Lasers (Cyberknife, Linac) } \\
\text { 1) A pre-treatment check of the orthogonality of the laser } \\
\text { system as its alignment with respect to the isocentre should } \\
\text { be checked. If out of limits, it should be adjusted within } \\
\text { limits before treatment. } \\
\text { Immobilisation } \\
\text { 2) Assure correct and rigid positioning of the frame, e.g. } \\
\text { invasive or frameless mask, to the treatment couch. } \\
\text { 3) Take your time for fine-adjustment of target positioning to } \\
\text { the isocentre. } \\
\text { 4) The people doing this should be trained that the fine- } \\
\text { adjustment might be time consuming while a pressure is } \\
\text { there "to act quickly to be patient friendly". } \\
\text { Rontgen-Light field (Linac) } \\
\text { 5) Patient alignment by light field alone has to be considered } \\
\text { as insufficient with respect to target alignment at isocentre. } \\
\text { Frame-based alignment, e.g. invasive or frameless, should }\end{array}$ \\
\hline
\end{tabular}

\footnotetext{
${ }^{7}$ Rule of thumb: $1 \mathrm{mrad}$ is equal to $1 \mathrm{~mm}$ deviation at $1 \mathrm{~m}$ distance.
} 


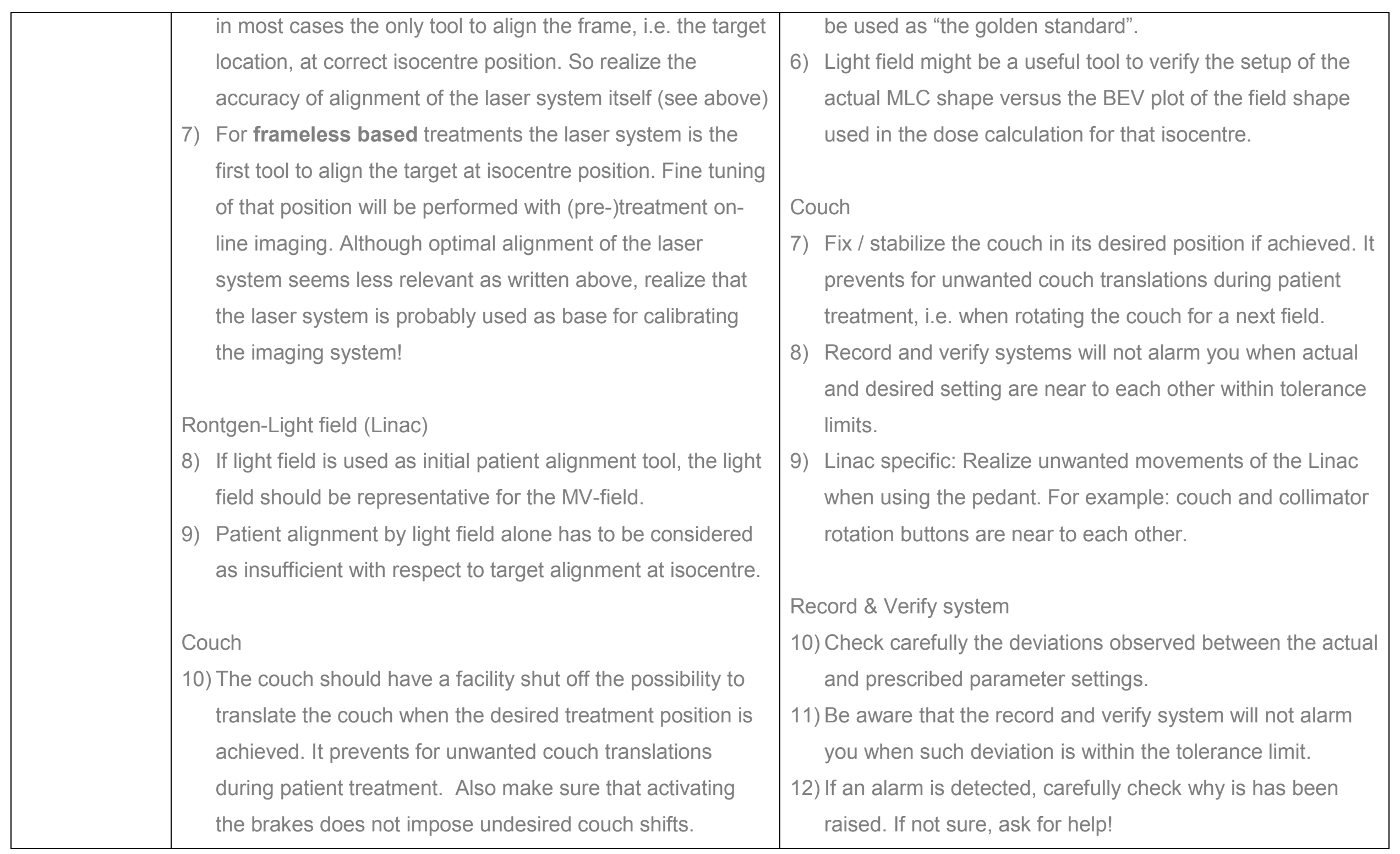




\begin{tabular}{|c|c|c|}
\hline & $\begin{array}{l}\text { 11) Linac specific: One way to do is to shut off the pendant } \\
\text { facility for couch translations. } \\
\text { 12) Gamma Knife specific: Investigate the accuracy in frame } \\
\text { positioning with respect to the helmet. A regular (pre- } \\
\text { treatment) quality control should be present. The couch } \\
\text { itself is not the problem } \\
\text { Record and Verify system } \\
\text { 13) Investigate the deviations, i.e. the tolerance limits, allowed } \\
\text { between actual and prescribed parameter setting. } \\
\text { 14) In practice they will be a trade-off between desired } \\
\text { accuracy and manageable treatment performance. } \\
\text { 15) Note that within this limits the record and verify system will } \\
\text { not alarm you about an observed deviation. } \\
\text { Chapter 11: more aspects about quality control have been } \\
\text { given. }\end{array}$ & $\begin{array}{l}\text { 13) In case of fractionated treatment, couch parameters } \\
\text { obtained in the first fraction can help you to re-align the } \\
\text { target to the isocentre. } \\
\text { 14) In the case of more isocentres / targets: } \\
\text { a) Be careful to take the correct treatment plan for the } \\
\text { target positioned at the isocentre. } \\
\text { b) Independent calculation of the couch parameters per } \\
\text { isocentre and verification with actual patient / couch } \\
\text { position is recommended. }\end{array}$ \\
\hline
\end{tabular}




\begin{tabular}{|c|c|c|}
\hline & \multicolumn{2}{|c|}{ Treatment Positioning: Imaging systems } \\
\hline Aspect & Methodology and Equipment (Link to Figure 2B) & Process chain (Link to Figure $2 \mathrm{C}$ ) \\
\hline Quality Control & $\begin{array}{l}\text { CT-kV-(ExacTrac) - MV-mech. Isocentre)) } \\
\text { 1) Treatment and imaging isocentre should coincide } \\
\text { a) Request for an high accuracy, i.e. } \varnothing \leq 1.0 \mathrm{~mm} \text {. } \\
\text { b) This should be tested and optimized for every used } \\
\text { combination. } \\
\text { 2) If long term stability cannot be assured, a quick check } \\
\text { protocol should be there to assure the accuracy just before } \\
\text { treatment, e.g. ball-bearing test. } \\
\text { QA protocols } \\
\text { 3) QA protocols should be robust, easy and fast. }\end{array}$ & $\begin{array}{l}\text { CT / kV [123, 145, 149] } \\
\text { 1) Test daily whether the treatment and imaging isocentre } \\
\text { coincide; } \\
\text { a) Run tests at different gantry angles. } \\
\text { b) If criteria are not met, optimize the system. } \\
\text { 2) Pre-treatment position verification: } \\
\text { a) Always preform positioning verification based on } \\
\text { planning CT / MRI or DRR.. } \\
\text { b) In case of multiple targets, perform a check per } \\
\text { isocentre. } \\
\text { Gamma Knife specific. } \\
\text { Pre-treatment imaging in treatment position per individual } \\
\text { patient treatment is not possible with older versions. So: } \\
\text { 1. Although frame movement based on coordinate shift } \\
\text { procedure is motorized and in principle accurate, there } \\
\text { should be a method available to convince yourself of the } \\
\text { correct positioning of the frame for each individual patient } \\
\text { treatment. } \\
\text { 2. Be aware of motor-slipping: it is possible without } \\
\text { observation and warning. }\end{array}$ \\
\hline
\end{tabular}




\begin{tabular}{|c|c|c|}
\hline Add-ons & $\begin{array}{l}\text { Statement: For Linacs: avoid add-ons where other options are } \\
\text { available. } \\
\text { Main reason is: } \\
\text { - The outlining of such devices with respect to beam focus } \\
\text { and central beam ray line might cumbersome to fulfil each } \\
\text { treatment moment } \\
\text { The safety to use the correct device, e.g. cone, or having } \\
\text { correct connection, e.g. MLC, during individual patient } \\
\text { treatment } \\
\text { If an add-on is required, e.g. cones / aperture systems for } \\
\text { Cyberknife, Gamma Knife and specific Linac-treatments: } \\
\text { 1. Convince yourself the reproducibility in re-positioning } \\
\text { accuracy of that device. } \\
\text { 2. Set-up a method to assure yourself as much as possible } \\
\text { the right device is used during individual patient treatment. } \\
\text { (risk analysis). } \\
\text { 3. If an add-on MLC is used on a Linac, setup a QA program } \\
\text { to assure accurate alignment of the add-on MLC leafs with } \\
\text { respect to the collimator rotation axis; in general such a } \\
\text { program is much more complex compared to cone-testing. }\end{array}$ & $\begin{array}{l}\text { In case an add-on is required, e.g. cones / aperture systems } \\
\text { for Cyberknife, Gamma Knife and specific Linac-treatments: } \\
\text { Cones / Aperture system (Cyberknife, Gamma Knife) } \\
\text { 1. Convince yourself the correct cone / aperture is are used. } \\
\text { Cones (Linac) } \\
\text { 1. Convince yourself the correct cone is used. } \\
\text { 2. A bar-code system might be helpful. } \\
\text { 3. Realize that the jaws are at correct position; often that } \\
\text { setup is not automated! So: check it! } \\
\text { 4. Convince yourself the outlining of the device is sure just } \\
\text { before patient treatment; inaccuracies depends on fixation } \\
\text { construction of the cone into the treatment head. Checking } \\
\text { on correct positing might be possible by using a dial } \\
\text { indicator. } \\
\text { Linac, add-on MLC: } \\
\text { 5. Realize that field-shape and orientation can be checked } \\
\text { easily with light field projection on a paper positioned at } \\
\text { the isocentre. }\end{array}$ \\
\hline
\end{tabular}




\begin{tabular}{|c|c|c|}
\hline & \multicolumn{2}{|c|}{ Treatment Delivery: Quality Control \& Irradiation } \\
\hline Aspect & Methodology and Equipment (Link to Figure 2B) & Process Chain (Link to Figure 2C) \\
\hline General & $\begin{array}{l}\text { The mechanical and dosimetrical aspects of the treatme } \\
\text { process control system, as described by Figure 2D. } \\
\text { As described there, the best way is to perform an "end-t } \\
\text { given in Chapter } 7 \text {. } \\
\text { Without being complete, below several basic advices are }\end{array}$ & $\begin{array}{l}\text { t delivery as the actual irradiation of a patient can be incorporated in a } \\
\text {-end" test. Additional technical information about the end-to-end test is } \\
\text { summarised. }\end{array}$ \\
\hline $\begin{array}{c}\text { Mechanical } \\
\qquad \& \\
\text { Dosimetrical }\end{array}$ & $\begin{array}{l}\text { 1) Define the basic aim of your test: } \\
\text { a) Following the setup of this report, here it should } \\
\text { be a technical investigation of well-working of } \\
\text { your system. } \\
\text { b) Keep in mind this test should be in relation to } \\
\text { the aim of the "end-to-end" used in the "process } \\
\text { control". } \\
\text { 2) Setup "on paper" the aims of your test. } \\
\text { a) Realize that in that way you might have several } \\
\text { "end-to-end" tests, e.g. target-type treatment } \\
\text { dependent, several isocentres in a single } \\
\text { patient. } \\
\text { b) An "end-to-end" test can never replace standard }\end{array}$ & $\begin{array}{l}\text { 1) Define the basic aim of your test: } \\
\text { a) Following the setup of this report, here it should be a process } \\
\text { control of well-working of the treatment as performed by all } \\
\text { disciplines involved in stereotactic treatment (see nearby). } \\
\text { b) Keep in mind this test should be in addition to the aim of the } \\
\text { technical "end-to-end" test. } \\
\text { 2) Setup "on paper" the aims of your test. } \\
\text { a) Note that technical performance is tested separately, so focus } \\
\text { on "process control". } \\
\text { b) Process control means here whether each discipline is doing } \\
\text { c) Nis / her job well. } \\
\text { "doing your job well": protocols only help you but }\end{array}$ \\
\hline
\end{tabular}




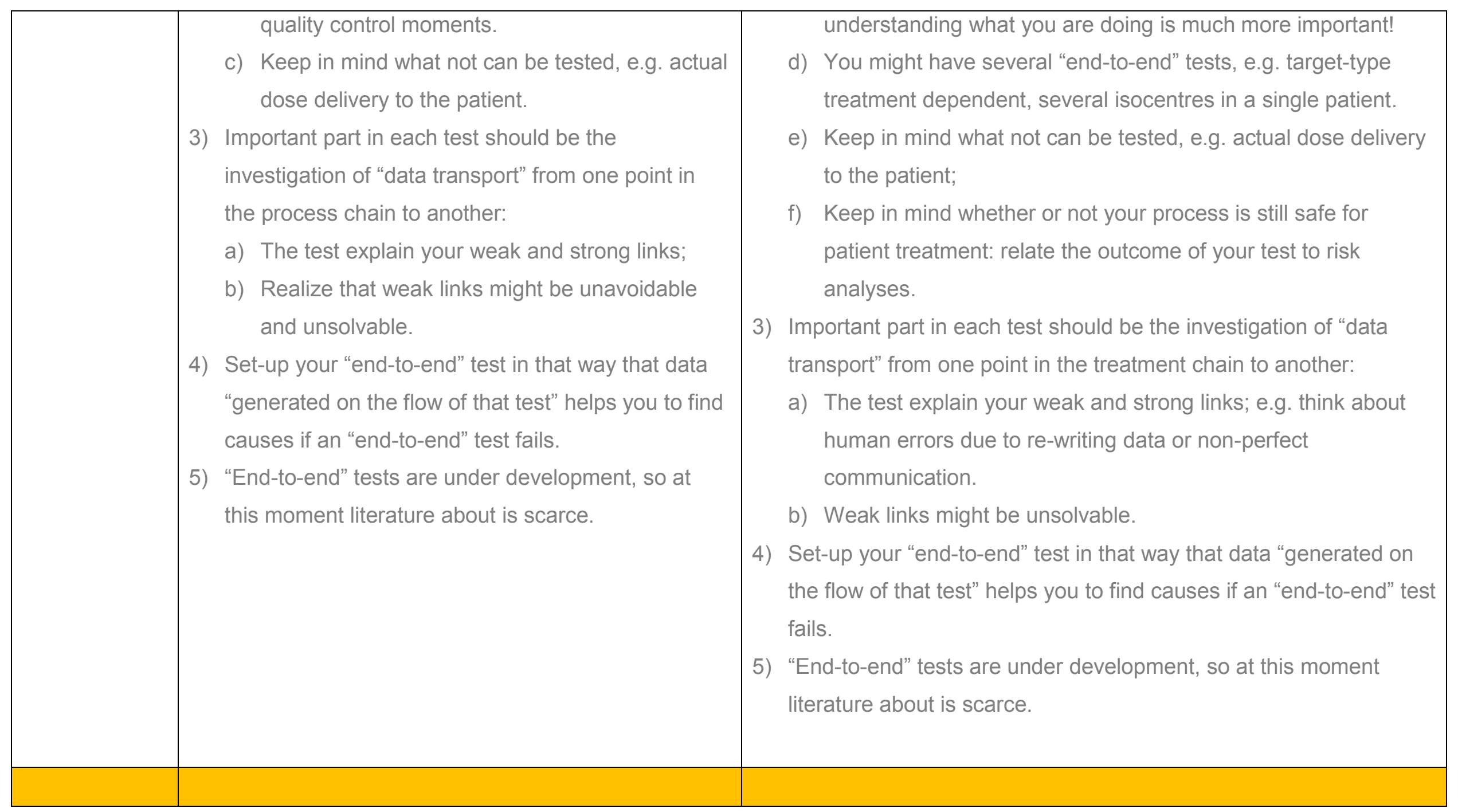




\section{Appendix: Process control (Figure 2D)}

In this Appendix detailed information is given on the subject "Process Control" (Figure 2D) Note: in chapter 6-Appendix , "Treatment Delivery, Quality Control \& Irradiation” several basic aspects regarding the end-to-end test have been presented.

\subsection{End-to-end test: phantom}

Phantoms to be used in an end-to-end test can be provided by the manufacturer delivering the stereotactic equipment or by others companies. If not, such phantom has to be constructed by the institution itself or obtained in another way.

The phantom used might influence the dose detection method to be used. As stereotactic treatment is a high-fraction dose high precision target positioning treatment, such dose detection system should have a high-spatial resolution and a high-accuracy in dose detection in the high-dose region.

In addition, the phantom used should be flexible since different inserts may be required for scanning on different imaging devices (MRI, CT) or to mimic different geometries. Despite this flexibility, the geometrical accuracy should be high if the phantom is to be used in a meaningful end-to-end test.

If a single phantom does not fulfil the criteria stated above, complementary phantoms should be used.

\subsection{End-to-end test: dose detector}

\subsubsection{Detector type}

The first step in an end-to-end test is to investigate the dose distribution around target and critical organs. The second step is to investigate the absolute dose delivery. The detectors should adhere to the requirements of the test.

High-spatial resolution and a high-accuracy in dose detection is required in stereotactic treatment verification, i.e. at least at $\mathrm{mm}$-level and $5 \%$ overall dose accuracy, respectively.

The latter means that the accuracy of the detector system is enough to discriminate a $5 \%$ differences between measurement and dose calculation as significant. 
Dose distributions: at the moment film and portal imaging devices are only usable as relative detector system. (Small) ionisation chamber, TLD and alanine are well-established standard devices. Good knowledge and skills with a dose detector system used is mandatory, but what and how is beyond the scope of this report. More details are given in chapters $8 \& 9$.

\subsubsection{Film.}

At present, Gafchromic film is the most promising film which can be used. It can be easily handled and modelled into structures which can be positioned in the phantom.

\subsubsection{Portal imaging device.}

As the dose distribution in the phantom has to measured, the portal imaging detector should be always positioned behind the phantom, to incorporate the transmission and scatter behaviour of the irradiation photon beam in the phantom.

An accurate algorithm should be available to transpose the detected dose distribution on the detector into a dose distribution in the phantom. At the moment such software is only available at a limited number of institutions.

\subsubsection{Pre-treatment verification: phantom}

In contrast to the end-to-end test, the phantom used in the pre-treatment verification may be limited to the geometry under investigation. Since pre-treatment verification often investigates the absolute dose and dose distribution on details, a high dosimetric and spatial accuracy is required.

\subsubsection{Pre-treatment verification: detector}

About the dose detector, similar aspects have to be considered as for the end-to-end test.

\subsubsection{Pre-treatment verification: acting}

Control of the treatment planning beam data is the first step in any end-to-end test. It can easily be done by setting up simple fields on a CT-scanned phantom. Be aware of resolution and choice of detector; be independent of data and means used in gathering treatment planning input data.

\subsubsection{More detailed information: see Chapter 8 \& 9 .}




\section{Appendix: Small field dosimetry: detectors}

This Appendix briefly summarizes aspects of small field dosimetry, related to detectors [50, $65,137,138,154,159]$.

\subsection{Introduction}

In traditional radiation therapy field sizes typically range from $4 \times 4 \mathrm{~cm}^{2}$ to $40 \times 40 \mathrm{~cm}^{2}$ and the clinical dosimetry systems have been developed accordingly. However, with the introduction of stereotactic radiotherapy extremely small fields in the order of few millimetres are introduced and both the dosimetry system's response as well as the applied dosimetry protocols require careful review. Small-field dosimetry is challenging due to the lack of electron equilibrium, size of detectors, variations in energy spectrum and associated dosimetric parameters and the composition of the medium. It should be noted that changes in lateral electron equilibrium are exasperating at higher energies. For a variety of reasons, including increasing uncertainty in output factors with decreasing field size and beam perturbation by tissue heterogeneities, small field irradiation using high-energy photon beams should be avoided.

With a large selection of radiation detectors and shapes the choice of a proper detector in small fields could be challenging especially in inhomogeneous media. This section will provide a generic overview of detector types, addressing the application for absolute dose, determination of output factors and beam profiles, and how to demonstrate end-to-end dosimetric capabilities in treatment delivery.

\subsection{Detector types, choices}

The choice of a detector is a critical consideration in stereotactic treatment beam characterisation [89]. Rice et al. [131] state that both the lack of lateral charged particle equilibrium and the steep dose fall-off in the penumbra of small fields, necessitate the use of small detectors. It has been suggested [e.g. 52] that the maximum inner diameter should be smaller than the beam radius. Various detectors have been developed to meet those requirements and although the use of one detector to acquire all the necessary data would be welcome, provided such a detector can be developed, Podgorsak [127] points out that it is 'good practice' to compare several different types in clinical practice and perform cross references. A good understanding of the detector's properties and limitations depending on field size and type of measurement being performed, is warranted. The reader is referred to 
the excellent references on the characteristics and applications of various detectors by Papas et al. and Ding et al. [48, 122]. Das et al. [38] provide a good review on the perturbation conditions and other issues associated with reliable measurements in nonstandard conditions. Candidate detectors covered briefly in this section include small ionisation chambers; semiconductors such as diodes, MOSFETS and diamond detectors; film dosimetry, both radiographic and Gafchromic, thermoluminescent detectors, alanine detectors and gel dosimetry.

\subsection{Ionization chambers}

As in standard radiation therapy, ionization chambers are the most common detector for collecting beam data in stereotactic treatment. Their dose response is nearly independent on energy, dose and dose rate; they exhibit excellent stability, linearity and reproducibility. For small field sizes care should be taken to ensure that the dimensions of the chamber's sensitive volume are considerably smaller than those of the radiation fields. Various manufacturers offer a wide range of chamber types (cylindrical, spherical and plane parallel), and a possible classification could be defined as a function of their sensitive volume [37].

Considering ionization chamber types:

- Standard farmer-type ionization chamber (active volume in de order of $10^{-1} \mathrm{~cm}^{3}$ ) has an active volume of on average $0.6 \mathrm{~cm}^{3}$.

- Mini ionization chamber (active volume in de order of $10^{-2} \mathrm{~cm}^{3}$ ): typical active volumes are $0.05 \mathrm{~cm}^{3}$.

- Micro ionization chamber (active volume in de order of $10^{-3} \mathrm{~cm}^{3}$ ): Typical active volumes are $0.007 \mathrm{~cm}^{3}$.

- Micro ionization chambers tend to react awkward for $10 \times 10 \mathrm{~cm}^{2}$ field sizes, related to over-response to low energy scatter. Therefore, when using this type of chambers it is recommended to reference them against the standard ionization chambers in $4 \times 4$ $\mathrm{cm}^{2}$ fields, and reference the $4 \times 4 \mathrm{~cm}^{2}$ against a $10 \times 10 \mathrm{~cm}^{2}$ with a standard ionization chamber (see below) [30, 81, 90, 106, 108].

\subsection{Diodes, MOSFETS and diamond detectors}

Diodes are available with relatively small sensitive volume with excellent spatial resolution making it ideal for obtaining small field beam characteristics, and being a solid state detector they feature high sensitivity compared to ionization chambers. The response of diode detectors depends on temperature, dose rate and energy, and depending on the design, 
some detectors show angular dependence as well [72]. In practice, shielding can be added to diode detectors to limit the large sensitivity for low-photons. As no simple answers can be given what to use and why, one should be aware of the difficulties around diode use in stereotactic beam measurements [31, 34, 36, 46, 52, 59, 81, 99] and one should be careful in using them.

Although a MOSFET is based on a similar semi-conducting technology, its way of use is different from diodes. Its use is complex while there is contradicting literature on dose rate, temperature and directional dependence. Careful cross calibration will be needed [146].

The small sensitive volume $\left(1.0-6.0 \mathrm{~mm}^{3}\right)$ and tissue equivalence of the diamond detectors makes it ideal for small field dosimetry and beam profile measurements. A major limitation is dose rate dependence and their high price compared with other solid state detectors as they are difficult to manufacture $[75,87,105,111,129]$

\subsection{Thermoluminescent dosimetry}

Thermoluminescent detectors (TLD) have a long state of service in radiotherapy, particular the small size and absence of electronic components are attractive for use in anthropomorphic phantoms and postal auditing [RPC, Followill]. TLD needs individual and regular calibration to take into account possible drifts of the relative individual sensitivity, which is influenced by the annealing process and ageing of the TL material. Bjarngard et al. reported a precision of $0.2 \%$, achievable using readings of 15 dosimeters for each dose point [23], but in general it is accepted that uncertainties of approximately $2-3 \%$ (1 SD) can be obtained in routine use, provided the read-out procedure is meticulously followed. The repeatability depends strongly on the combination of TL material chosen, type of TL-reader, as well as on the read-out cycle. For more information the reader is referred to Kerby et al., Derreumaux et al., Izewska et al., and Ferreira et al. [43, 60, 78, 83]

\subsection{Film dosimetry}

Basically, two types of films are being used in clinical practice. First, radiographic or silver halide-based film that require processing and have a strong energy dependence. The development and calibration process requires special attention in order to limit the uncertainties due to emulsion irregularities and film processing parameters. Second, Gafchromic films based on the transformation of monomers (positioned in crystals) into polymers. Those films don't need any development process. Due to its near-water 
equivalence it has no low-photon energy dependency when used in stereotactic megavoltage beams. The advantage of Gafchromic over radiographic film is evident. However, it should be noted that similar to radiographic film the accuracy in dose detection is critically dependent on the scanning process and the time between measurement and read-out [148] Also variations in film composition within films and between different batches have been observed much like those observed with radiographic film [29, 97]. The reader should be aware that at this moment conflicting results have been reported in literature concerning the accuracy in response variations with EBT, EBT2 and EBT3 Gafchromic film; the solution is not evident [120].

The excellent spatial resolution in addition to the planar aspects make film an ideal dosimeter for high-resolution, relative measurements. In addition, with careful processing and cross referencing with other detectors it can be used for defining output factors as well [115]. Although radiographic film was originally tested for output measurements, inconsistencies in the processing and general non-reproducibility diminished that particular use. Recently, some authors have investigated both radiographic and Gafchromic film in the measurement of relative outputs, with promising results. Somigliana et al [144] used both types of film and a diode to measure relative outputs on both a Gamma Knife unit and a Linac. All 3 detectors produced equivalent results on the Linac for collimator diameters between 24 and $14 \mathrm{~mm}$. On the Gamma Knife unit, differences were reported, probably because the directional dependence of the detectors becomes important due to the geometrical set-up of the ${ }^{60} \mathrm{Co}-$ sources.

\subsection{Gel dosimetry}

3D-gel dosimetry provides an excellent tool for end-to-end testing as it combines both localization as well as dosimetric verification. Unfortunately, the process of creating stable gels and anthropomorphic configurations is limited to experienced and specialized centres, which makes this tool rather cumbersome for basic dosimetry [21, 40, 41]. In addition, the majority of these gels require extensive use of MRI equipment for read-out, necessitating a multidisciplinary approach and close collaboration with MRI specialists. Mailed dosimetry systems are available (Medical Gel Dosimetry Systems, Inc. Madison), and efforts are being made to avoid MRI read-out by developing optical scanning methods. 


\subsection{Alanine dosimetry}

Particular about alanine/EMR (electro magnetic resonance) dosimetry is that the measurement performance for absolute dosimetry can be extrapolated to non-reference situations. Alanine dosimetry can be used in situations where, for pure technical reasons, the reference measurement conditions as specified in international codes of practice for absolute dosimetry, can't be met [15]. When using ionization chambers or solid state detectors for QA of small and irregularly shaped IMRT fields, the detector fluence-perturbation effect may invalidate the application of standard-field dosimetry protocols. These measurement complications reveal a deeper underlying problem: the presence of a physical detector causes a change in particle fluence in water at the point of measurement, compared to the fluence in absence of the detector. The presence of a solid detector, composed of water equivalent material with no additional components, avoids this complication. Alanine/ EMR dosimetry meets these criteria as radicals, formed in a tissue equivalent substance, can be measured directly with quantitative EMR spectroscopy [16]. Currently, read-out of these detectors is limited to a few specialized centres, but the detector is an excellent candidate for external auditing and end-to-end testing of radiosurgery programs [136]. 


\section{Appendix: Small field dosimetry: beam characteristics}

This Appendix briefly summarizes aspects of small field dosimetry, related to beam characteristics $[42,101,103,137,152,159]$.

\subsection{Introduction}

Ensuring dosimetric integrity relies on several elements: (1) basic characterisation of the treatment beams, which is particularly challenging due to the small field size, and (2) an adequate and correct commissioning of the beam data input for the treatment planning system.

In traditional radiation therapy field sizes typically range from $4 \times 4 \mathrm{~cm}^{2}$ to $40 \times 40 \mathrm{~cm}^{2}$ and the clinical dosimetry systems have been developed accordingly. However, with the introduction of stereotactic treatment / radiosurgery (SRS) and intensity-modulated radiotherapy (IMRT) extremely small fields in the order of few millimetres are introduced and both the dosimetry system's response as well as the applied dosimetry protocols require careful review. Smallfield dosimetry is challenging due to the lack of electronic equilibrium, size of detectors, variations in energy spectrum and associated dosimetric parameters, and the composition of the medium. It should be noted that changes in lateral electronic equilibrium are exasperated at higher energies.

For a variety of reasons, including increasing uncertainty in output factors with decreasing field size and beam perturbation by tissue heterogeneities, small field irradiation using highenergy photon beams should be avoided.

\subsection{Output factors, related to dosimetry systems used}

Adaptation (or removal) of flattening filters and the strong collimation to produce high dose rate narrow fields for stereotactic treatment modify significantly the energy spectra of conventional radiotherapy broad photons beams. Absolute dosimetry of the small photon fields with ionization chambers is constrained by the lack of charged particle equilibrium in the radiation field and the size of the detector, invalidating the application of open-field dosimetry protocol data for the derivation of absorbed dose to water from ionization chamber measurements $[6,10,13,63,64]$. The chamber's sensitive volume effect can be solved in part by the development of micro ionization chambers, but considerable uncertainty exists with regard to the validity of using existing dosimetry data with such chambers. Basic data 
(e.g. stopping-power ratios) have been obtained for broad beam configurations where conditions of quasi-electron equilibrium prevail. It is, however, questionable that such data can be used in the dosimetry of narrow fields while keeping the uncertainty at the same level as for the broad beams used in the reference conditions of a clinical accelerator calibration. To accurately measure dose in small fields it is important to realize that most of the parameters that are used in the dosimetry protocols (e.g. mass attenuation coefficients, stopping power ratios, beam quality factors, ...) are depending on the energy spectrum. Hence, comparing dose measurements in reference fields $\left(10 \times 10 \mathrm{~cm}^{2}\right)$ with measurements in small fields is uncertain, especially for fields in specialized treatment machines such as NOVALIS, Cyberknife and TomoTherapy. A careful selection of detectors and understanding of their response in small fields is warranted; see Chapter 8.

In clinical practice, however, studies on stopping-power ratios have shown that the uncertainty due to narrow field dosimetry is at the same level as for broad beam dosimetry. More particularly Sanchez-Doblado et al. have shown that for water/air and PMMA/air stopping-power ratios agree within $\pm 0.3 \%$ with reference (i.e. $10 \times 10 \mathrm{~cm}^{2}$ ), well within the estimated standard uncertainty of the reference stopping-power ratios (i.e. 0.5\%) [134, 139]. It is important to realize that those studies have been performed on conventional treatment machines (e.g. Elekta Sli15: $D_{20} / D_{10}=0.58 @$ UZ Brussels) with extra collimation to obtain narrow beams. Some recent developments, however, are introducing clinical accelerators with flattening filters adapted for narrow fields (NOVALIS, BrainLAB: $D_{20} / D_{10}=0.56 @$ UZ Brussels) or even omitted the flattening filter all together (TomoTherapy, TomoTherapy Inc.: $D_{20} / D_{10}=0.52 @$ UZ Brussels) as the device is designed for IMRT and the concept of flat homogeneous treatment fields becomes irrelevant. The latter translates to an increased uncertainty in the beam quality parameter (e.g. $\mathrm{k}_{\mathrm{Q}}$ in the TG51 dose-to-water protocol) [80, 121]. The dosimetry protocols can be extended by including an additional measured-toreference factor, $\mathrm{k}_{\mathrm{mr}}$, which has a similar role as the quality conversion factor, $\mathrm{k}_{\mathrm{Q}}$, but it transfers the dose value from the measurement to reference calibration conditions, rather than from a beam of one quality to another. Jeraj et al. showed that for helical TomoTherapy this conversion is close to unity for larger field sizes, however, for smaller fields $\left(2 \times 2 \mathrm{~cm}^{2}\right)$ this factor accounts for an approximately $1 \%$ correction [80].

Recently the IAEA and AAPM proposed a new formalism for the dosimetry of small and composite fields with the intention to extend recommendations given in conventional Codes of Practice for clinical reference dosimetry based on absorbed dose to water. This formalism 
introduces the concept of two new intermediate calibration fields: (i) a static machine-specific reference field for those modalities that cannot establish conventional reference conditions, and (ii) a plan-class specific reference field closer to the patient-specific clinical fields, thereby facilitating standardization of composite field dosimetry [12]. Especially users of dedicated radiosurgery devices (such as Cyberknife and Gamma Knife) are advised to consult this paper.

Determination of small field output factors is often the most challenging measurement encountered in stereotactic treatment physics. As output factors are directly correlated with the calculated dose, any error in measurement will correspond to an equivalent error in the delivered dose. Some of the most common errors include: (i) failure to properly align the detector with the beam's central axis, (ii) use of an inappropriate detector. A number of groups have investigated the relationship between detector type and resulting output factors $[48,73,74,108,122]$.

\subsection{Beam profiles}

Central axis profiles are best obtained by scanning (both PDD or TMR) in a motorized water phantom using small volume ionization chambers $\left(\leq 0.015 \mathrm{~cm}^{3}\right)$ for fields larger than $2 \times 2 \mathrm{~cm}^{2}$ and stereotactic diodes for smaller fields as detector of choice. Detector alignment with the beam axis presents most likely the most difficult practical challenge for very small fields. Small field PDD and TMR curves exhibit the familiar dependence on field size in that the slope at larger depths becomes increasingly shallow for larger field sizes. Therefore, it is good practice to overlay these curves for different field sized in a single plot to detect possible errors in experimental set-up.

Interesting to note is that when measuring beam profiles for narrow field configurations in SRS, the depth of $d_{\max }$ decreases as field sizes decreases. This antithesis to large open fields is not related to electron contamination but is in part due to the increasing effect of phantom scatter as field size increases and possibly also due to the effects of tertiary collimator scatter [19, 108, 131]. An appropriately small detector is also required for accurate assessment of off-axis profiles because detectors with too large volumes will produce artificially broad profiles. Even small volume ionization chambers have been shown to be questionable for this purpose [48, 108, 122]. Radiographic film can over-respond in the penumbra region of small photon beams, also contributing to some broadening. Gafchromic 
film might be a promising alternative, but the contradicting observations in literature warrant some caution.

\subsection{Practical recommendations}

Again, beam data characterization will require the use of multiple detectors, depending on field size and type of measurement being performed.

A wide range of detectors has been developed with excellent spatial resolution for depth dose and beam profile measurements. The measurement of the relative output factors, however, represents the largest problem because no detector is ideal and a comparison between different detectors (cross reference) highlights the weaknesses of each detector rather than offering an accurate value of the output factor. For non-tissue equivalent detectors the energy difference results in variations in response at small fields relative to the standard $10 \times 10 \mathrm{~cm}^{2}$ reference field. To circumvent this problem, the use of these detectors should be limited to measurements in which the field size differences produce little change in the energy spectrum.

In practice scatter factors for small fields can be obtained by cross referencing the detector's response to an intermediate size field at which an accurate ion chamber measurement can be obtained [108]. For example, the output factor of a $5 \mathrm{~mm}$ circular cone can be determined from cross referencing the measurement obtained from a diode to that from an ionization chamber (IC) as follows [58]:

$S_{c, p}(r=5 \mathrm{~mm})=\frac{D_{\text {diode }}(r=5 \mathrm{~mm}, d, S S D)}{D_{\text {diode }}\left(40 \times 40 \mathrm{~mm}^{2}, d, S S D\right)} \square \frac{D_{I C}\left(40 \times 40 \mathrm{~mm}^{2}, d, S S D\right)}{D_{I C}\left(100 \times 100 \mathrm{~mm}^{2}, d, S S D\right)}$

In general, small field dosimetry can be successfully performed provided the following recommendations are considered:

- A small volume detector should be used that has minimum polarity, energy, dose and dose rate dependence.

- Micro-ionization chambers and stereotactic diodes are best suited for small field dosimetry, provided signal-to-noise issues are evaluated.

- If the field size is small compared to the detector size and charged particle equilibrium could be compromised, measurements might be performed at larger source to surface distance with proper correction. 
- Energy spectrum does vary in small fields such as SRS and IMRT, however, its impact is not significant. Careful evaluation might be warranted for those delivery systems where flattening filters have been adapted or removed entirely.

- Stopping power ratios in small fields are relatively constant for most ionization chambers.

- A cross reference and verification of small field beam parameters should be carried out with at least one other independent detector. 


\section{Appendix: Commissioning TPS: controlling the data}

In this Appendix more detailed information is presented about commissioning the treatment planning system.

\subsection{General Remarks}

Commissioning a treatment planning system is reported by e.g. NCS15 [27]. The tests described in that report remain valid for a stereotactic treatment planning system. Tolerances of $2 \mathrm{~mm}$ and $2 \%$ are often quoted [27, 51, 130], except for field sizes below $1 \times 1 \mathrm{~cm}^{2}$ where 5$6 \%$ dose differences are tolerated. Most recommendations of NCS15 remain valuable for the stereotactic treatment planning system, but the distance to agreement for individual beams is reduced to $1 \mathrm{~mm}$ or better $[51,126]$. Please notice that important issues on TPS are also handled in Chapter 6 of his report.

\subsection{Dosimetric verification}

Once reliable beam data have been obtained and the stereotactic treatment planning system has been commissioned, a thorough dosimetric validation is required comparing calculation of the basic beam characteristics with measured data. Failure to reproduce beam data in simple geometrical conditions is an indication of a fundamental problem. In a next phase a systematic approach is advised starting with dosimetric verification of simple plans accompanied by measurements in phantoms and working towards more sophisticated cases applying measurements of composite beam configurations. A combination of detectors and phantoms will be required to verify both the absolute dose delivered to the target (ionization chamber, film, alanine, ...) as well as the overall dose distribution (film, 2D-arrays, 3D-gel, ...). End-to-end assessment of all aspects of the process, localization as well as dosimetric, is essential [155]. Standard dosimetric verification procedures are important yet incomplete when commissioning a treatment planning system for stereotactic radiotherapy. As a final note, this end-to-end verification should always be performed through the workflow as applied in clinic, i.e. using the record and verify system and phantom set-up by those people that perform the patient set-up in clinical routine.

\subsection{Monte Carlo calculations}

It has been argued that Monte Carlo (MC) simulations are more appropriate in those situations where measurements are rather difficult (e.g. conditions of non-equilibrium, buildup dose regions and tissue interfaces) and various reports have highlighted the usability of 
MC for small field dosimetry [17, 26, 38, 42, 135]. To quote Das, Ding and Ahnesjö [38]: "It is also expected that the Monte Carlo techniques will increasingly be used in assessing the accuracy, verification, and calculation of dose, and will aid perturbation calculations of detectors used in small and highly conformal radiation beams." Without reopening the discussion between measurement and simulation, it is fair to acknowledge that both approaches feature intrinsic uncertainties. In the case of MC, it is important to realize that the modelling of the clinical machine defines its accuracy, and in case of discrepancies between measurement and MC, cross referencing can be difficult. 


\section{Appendix: Quality Control: Mechanical}

This Appendix describes more information about quality control of treatment devices related to mechanical characteristics.

\subsection{General}

In general, a high level of mechanical accuracy should be attained for all stereotactic or high precision target positioning treatments, irrespective of the machine used to deliver the dose. Mechanical QA tests should be designed to establish and maintain the mechanical accuracy of a stereotactic or high precision target positioning system by means of measurable parameters. During the process of acceptance of the equipment the supplier demonstrates its performance to the satisfaction of the customer against specifications, which should be part of the agreed contract. The mechanical measurements should (at least) satisfy the agreed upon specification values. Acceptance testing set therefore the baseline for future measurements and verifies that the equipment is mechanically functional and operates within certain tolerances from absolute specified values.

Published protocols contain tables with specified values defined as tolerance and/or action levels for the performance of system parameters. In the following paragraphs, depending on the treatment technique, specific protocols from the literature are suggested.

If a change in the baseline parameter exceeds the tabulated values in these protocols, action is required. Actions should be set by the supervising medical physicist in terms of the level of action (inspection, scheduled or immediate stoppage) to be taken and under what circumstances. The actions should be well known by all personnel involved in the QA process [84]. Definitions of tolerance and action level can be found in the glossary at the end of this report.

The frequency of mechanical performance tests is often suggested in published protocols. Daily tests must be scheduled prior to the first SRS/T treatment of the day, or in some cases prior to every treatment if equipment is removed from the treatment device (e.g. Linac: cones, add-on MLC). For other, less frequent tests, testing at less than the minimum frequency is permissible only if experience has established that the parameters of interest are highly stable. Documentary evidence supporting this decision is essential [20]. 


\subsection{Linac based techniques}

Extensive QA recommendations and guidelines can be found in literature for a stereotactic Linac based systems. In this report we suggest the use of the following published protocols, containing tables with tolerances for mechanical parameters as well as the frequency of the measurement to be performed:

- AAPM TG42 Report No 54: Stereotactic radiosurgery [141]

- AAPM TG142 Report: QA of medical accelerators [84]

- Canadian Association of Provincial Cancer Agencies (CAPCA): Standards for quality control at Canadian radiation treatment centres: Stereotactic Radiosurgery I radiotherapy [20]

In stereotactic treatment, machine and patient safety is also an issue as large doses are given in one or few fractions. Interlock functionality is therefore of highly importance. Additionally, a treatment plan with a possible risk for collision should be tested prior to the (1st) treatment in similar conditions. This is particularly helpful when using tertiary collimators (e.g. cones) in combination with computer controlled motion of the Linac (arc treatment). If necessary, the treatment plan needs to be adjusted.

\subsection{Cyberknife}

At the time of publication of this report, the only clinically implemented robotic radiosurgery device is the Cyberknife $®$. Recommendations and guidelines regarding mechanical alignment (Cyberknife) and QA of this robotic system can be found in recent literature:

- $\quad$ AAPM Task Group 135, Quality Assurance report for robotic radiosurgery [47].

It includes examples of checklists for daily, monthly, annually and upgrade $Q A$ as guidance for medical physicists as well as a description of tests to be performed and tolerances to be attained. In Chapter 12, Glossary Cyberknife, several terms used have been described.

\subsection{Gamma Knife}

\subsubsection{Introduction}

The Gamma Knife is a fully integrated stereotactic system, with less degrees of freedom than a linear accelerator or the Cyberknife ${ }^{\circledR}$. With respect to machine specific requirements, the Gamma Knife therefore needs more integral QA tests, rather than mechanical component based tests. 
Unfortunately, literature data concerning Gamma Knife mechanical quality assurance is rather sparse [e.g. 104], especially for Gamma Knife types B, C and 4C. Therefore, this report gives some suggestions, without the intent to be complete! The advised tests and tolerance levels listed in Table I are a combination of those recommended by the acceptance and commissioning testing procedures of the manufacture, scarce scientific literature and good physics practice.

Some years ago the Gamma Knife manufacturer (Elekta Instruments AB, Stockholm, Sweden) introduced a new type named Perfexion. The Perfexion is different in design in such a way that it no longer has a separate automatic positioning system (APS), but the patient positioning is integrated in the table design. Also, the trunnion-mode no longer exists in this design and the helmets are integrated in the head in such a way that the sources move from one collimator section to the other. These differences have consequences for the QC of the Gamma Knife. More recent literature is available for this type of Gamma Knife [96]. In Chapter 12, Glossary Gamma Knife, several terms used have been described. Regarding the announced newest version of the Gamma Knife with integrated cone beam CT, no literature was available at the time out of writing.

\subsubsection{Machine Interlocks}

The Gamma Knife is equipped with several machine interlocks. Door interlocks, emergency switches, radiation lights, etc. should be tested on a regular bases, equivalent to procedures of linear accelerators. Other Gamma Knife specific interlocks such as patient arm rest interlocks, should also be tested on a regular basis but at least once a year.

\subsubsection{Collision tests}

In practice it is possible to plan a treatment shot in such a way that the patients head, or the fixation frame, collides with the helmet. Possible shots that may cause a problem, are recognized by the planning system. These coordinates should be checked prior to treatment, with a dummy run.

\subsubsection{Helmet tests}

Each helmet provides a couple of safety checks. The helmet ID is recognised by the console, by means of micro switches. For the Perfexion model the helmet does not change. However, the placement of the source with respect to the helmet does. Therefore this should be verified in some way. 
The patients hair is protected by a helmet cap, which needs to be on the helmet before the treatment can start. Finally, two micro switches are on the top and bottom of the helmet. When docking the patient in the gantry these switches must be activated within a certain time from each other, and within a certain distance of the inner collimator.

Each can be tested with help of the test box from the manufacturer that indicates ID, cap sensor and distance switches with LED's. These can be adjusted if necessary. For the distance switches an adjustment ring can be placed over the helmet. This will activate the micro switches. The test tool will indicate activation of both switches with a green LED. By introducing shims of different thickness between the helmet and the adjustment ring the switches can be checked. The switch should get inactive at $0.1 \mathrm{~mm}$ shim, indicated by a red LED on the test box. According to the manufacturer this test should be performed weekly

\subsubsection{Radiation safety tests}

Since a Gamma Knife holds permanent radiation sources within, an independent radiation alarm should also be operational if no patients are being treated. Regular wipe-tests, radiation surveys and radiation leakage tests should be performed. Mostly these frequencies prescribed by legislation or legislation controlling offices.

\subsubsection{Patient positioning}

The patient should be positioned relative to the isocentre of the sources of the Gamma Knife with an sub-millimetre accuracy. This is dependent on the frame stability, the image and coordinate definition accuracy and the machine accuracy. This section will focus on the machine accuracy specifically.

As mentioned there are two different ways of positioning the patient, one by hand and one automatic. Since both systems are used on the same Gamma Knife, each system needs to be checked on accuracy.

\subsubsection{APS}

The automatic system can be checked by simply reading the rulers on the APS. The accuracy of the system is said to be $<0,2 \mathrm{~mm}$. Left and right APS have separate motors. Inconsistencies between left and right position can be detected by the system itself. It is advisable to do an additional visual check in case there are known problems. 
The Gamma Knife has a build-in protocol for a daily positioning consistency check, to be performed with a tool delivered with the system. The test tool has a spring loaded tip. The APS is instructed to move to two opposite peripheral points. When each point is reached the tool is pressed against the helmet. Markers on each of the helmets and on the tool provide a measure for consistency. The reading should be within $0.5 \mathrm{~mm}$ of previous readings. The manufacturer advises to do this after each APS trunnion exchange. In clinical practice this means about every day and sometimes more than once a day. This might however be a bit on the conservative side and is very time consuming. It is advisable to start these checks on a daily basis, but if the user is confident that the system is reproducible even after switching between APS and trunnions, the frequency can be brought down to once a week.

There is a more extended protocol for the APS that consists of 50 positions. This protocol is only used by the manufacturer, on each maintenance. It is recommended that it should be performed once a year, or on every switch or repair on the APS system.

\subsubsection{Trunnions}

The trunnions are basically slide rulers that can be attached to the patient frame, in order to obtain $y$ and $z$ coordinates, and to the helmet for the $x$ coordinate. The $x$ coordinate can be checked by using a tool that is delivered with the Gamma Knife. This $X$ shaped tool is attached to the helmet and the $x$-coordinate slider is pushed to the centre of the tool. The position then should be $x=100$. The manufacturer states that this check should be done weekly, while the trunnions are in use, and whenever damage is expected. However, this test only checks for $x$ coordinates, only in one position, and not for $y$ and $z$ coordinates. And no alternative for these is provided. A more meaningful test can be performed by simply checking if left and right coordinates are the same, when the patient is mounted. This can be done for every patient that has trunnion co-ordinates.

Also trunnions can be checked by comparing it to a slide ruler. This is only advised in case of suspected deformation.

\subsubsection{Patient Positioning System (PPS)}

In the Gamma Knife Perfexion model the helmet and positioning systems are integrated into one patient positioning system. Overall accuracy of this system should be within $0.3 \mathrm{~mm}$. As the system no longer has outside reference points checking accuracy of the positioning alone 
will be much more difficult. Most likely the best option will be to check this with a film measurement, together with radiation isocentre checks. 


\begin{tabular}{|c|c|c|c|c|}
\hline Overview Gá & ma Knife QC tests: minimum requirements & & & \\
\hline QC Test & Description & $\begin{array}{l}\text { Tolerance } \\
\text { Action level }\end{array}$ & Frequency & Comment \\
\hline Collision tests & & functional & Pre-treatment & \\
\hline Trunnion & & $<0.3 \mathrm{~mm}$ & Treatment & Perfexion model \\
\hline APS & View difference APS coordinate with ruler & $<0.2 \mathrm{~mm}$ & Treatment & \\
\hline PPS (moving table) & Overall & $<0.3 \mathrm{~mm}$ & & See advices manufacturer \\
\hline Overall patient positioning & & $<1 \mathrm{~mm}$ & & See advices manufacturer \\
\hline Machine interlocks & & functional & & See advices manufacturer \\
\hline APS & Predefined position reproducibility & $<0.05 \mathrm{~mm}$ & Weekly & 2 positions; extended test X-frame \\
\hline Helmet tests & & $1 \mathrm{~mm}$ & 6 monthly & \\
\hline Isocentre check & Film measurement & $<0.3 \mathrm{~mm}$ & 6 monthly & \\
\hline Timer accuracy & Compared to external timer & $<0.2 \%$ & 6 monthly & \\
\hline Timer linearity & Increasing treatment time & $<0.2 \%$ & 6 monthly & \\
\hline Trunnion & & $<0.1 \mathrm{~mm}$ & Annually & \\
\hline Radiation safety check & & No leaks & Annually & According to legislation \\
\hline APS & Predefined position reproducibility & $<0.05 \mathrm{~mm}$ & Annually & 2 positions; extended test X-frame \\
\hline Frame stability & Revision of fixation device & & Per 3 years & \\
\hline
\end{tabular}




\section{Appendix: Descriptions and Glossary}

The list concerns descriptions and a glossary of terminology used in stereotactic treatment. The latter is separated to Linac, Gamma Knife and Cyberknife. The first column gives the description. The second column gives additional information.

The authors are aware the list will not be complete; new terminology will be introduced in the mean time writing this report.

\begin{tabular}{|c|c|}
\hline \multicolumn{2}{|l|}{ Definitions } \\
\hline \multicolumn{2}{|l|}{ Accelerated fractionation } \\
\hline $\begin{array}{l}\text { Accelerated fractionation is a dose delivery strategy } \\
\text { with a similar number of fractions compared to } \\
\text { conventional fractionation schedule but delivered } \\
\text { over a shorter time period. Usually it is accompanied } \\
\text { with a reduced fraction dose to maintain the same } \\
\text { Biological Equivalent Dose (BED) [e.g. 57] }\end{array}$ & $\begin{array}{l}\text { a. Typical example is the treatment strategy with two fractions a day. } \\
\text { b. The overall length of treatment time reduces. } \\
\text { c. See the definition of hyper-fractionation } \\
\text { d. See the definition of hypo-fractionation }\end{array}$ \\
\hline \multicolumn{2}{|l|}{ Acceptance } \\
\hline $\begin{array}{l}\text { Acceptance is the procedure in which the customer } \\
\text { takes over the equipment from the vendor and } \\
\text { becomes owner of that equipment. }\end{array}$ & $\begin{array}{l}\text { a. In general, before take over the equipment, customer and vendor test the equipment } \\
\text { on its functioning versus specifications. } \\
\text { b. If all specifications are fulfilled a "take over" document is signed by vendor and } \\
\text { customer. }\end{array}$ \\
\hline \multicolumn{2}{|l|}{ Action level } \\
\hline The action level is defined as an accuracy limit that if & a. If the ideal response is not immediately possible, then the use of the equipment must \\
\hline
\end{tabular}




\begin{tabular}{|c|c|}
\hline $\begin{array}{l}\text { a deviation between the actual status of a parameter } \\
\text { and its ideal status exceeds that accuracy limit a } \\
\text { response is required immediately in order to bring } \\
\text { the value back to or below the tolerance level. }\end{array}$ & $\begin{array}{l}\text { be restricted to clinical situations in which the identified inadequate performance is of } \\
\text { no significance. } \\
\text { b. The decision on the most appropriate response is made by the supervising experts } \\
\text { in consultation with the users of the equipment and others as appropriate. } \\
\text { c. The ideal response is to bring the system back to a state of functioning which meets } \\
\text { all tolerance levels before the system is re-introduced in the clinical process. } \\
\text { d. A clinical system with parameters which exceeds action levels should not be used } \\
\text { for patient treatment as there is no guarantee of a safe and proper treatment of the } \\
\text { patient. }\end{array}$ \\
\hline \multicolumn{2}{|l|}{ Commissioning } \\
\hline $\begin{array}{l}\text { Commissioning is the procedure in which data about } \\
\text { (the functioning of) the equipment is gathered } \\
\text { additional to those obtained during acceptance. }\end{array}$ & $\begin{array}{l}\text { a. Commissioning is supplied after the acceptance is finished. } \\
\text { b. Reason is that in that situation the equipment can be investigated on working like it } \\
\text { would be function in daily use. } \\
\text { c. As commissioning is supplied after acceptance, the commissioning and its validity is } \\
\text { the responsibility of the customer. } \\
\text { d. In general: if functioning of equipment is out of specifications but checked or } \\
\text { investigated after acceptance, the consequences are up to the customer. }\end{array}$ \\
\hline \multicolumn{2}{|l|}{ Competent (unconscious, incompetent) } \\
\hline $\begin{array}{l}\text { Competence is the capacity to act adequate, } \\
\text { efficient and effective, based on knowledge, skills } \\
\text { and experience. }\end{array}$ & $\begin{array}{l}\text { a. Competence should not be exchanged with qualification: a person can be competent } \\
\text { but not qualified to act. And revers: be qualified but not competent. }\end{array}$ \\
\hline
\end{tabular}




\begin{tabular}{|c|c|}
\hline \multicolumn{2}{|l|}{ Conformity Index } \\
\hline $\begin{array}{l}\text { The conformity index is defined as the quotient of } \\
\text { the Treated Volume (TV) and Planning Target } \\
\text { Volume (PTV), if the TV fully encompasses the PTV } \\
{[4,5,32,961,85,112,118,119,140,156,162]}\end{array}$ & $\begin{array}{l}\text { a. The limitations of this definition are reported by Loïc Feuvret et al [61]. However, in } \\
\text { this report we want to be consistent in definition with international reports. } \\
\text { b. The treated volume is the tissue volume that receives at least the dose selected and } \\
\text { specified by the radiation oncology team as being appropriate to achieve the } \\
\text { purpose of the treatment, i.e. cure, local target control or palliation. } \\
\text { c. According to this definition a value of } 1.0 \text { means ideal dose conformation to the } \\
\text { target, taking into account point a. }\end{array}$ \\
\hline \multicolumn{2}{|l|}{ Conventional fractionation schedule } \\
\hline \multicolumn{2}{|l|}{$\begin{array}{l}\text { Conventional fractionation schedule is considered as } \\
\text { a curative or local target control dose fractionation } \\
\text { schedule of } 1.8-3.0 \mathrm{~Gy} / \text { fraction, delivered at five } \\
\text { days a week. }\end{array}$} \\
\hline \multicolumn{2}{|l|}{ Co- registration } \\
\hline $\begin{array}{l}\text { Co-registration is a method to overlay one patient } \\
\text { dataset to another with the aim to identify identical } \\
\text { points in both datasets. }\end{array}$ & $\begin{array}{l}\text { a. In general the overlay is based on a rigid match, i.e. both patient datasets are not } \\
\text { deformed in that match procedure. } \\
\text { b. The overlay yields an overlaid-image dataset } \\
\text { c. See definition of registration. }\end{array}$ \\
\hline \multicolumn{2}{|l|}{ Coverage Index } \\
\hline The coverage index is defined as the ratio of the & ans no under-dosa \\
\hline
\end{tabular}




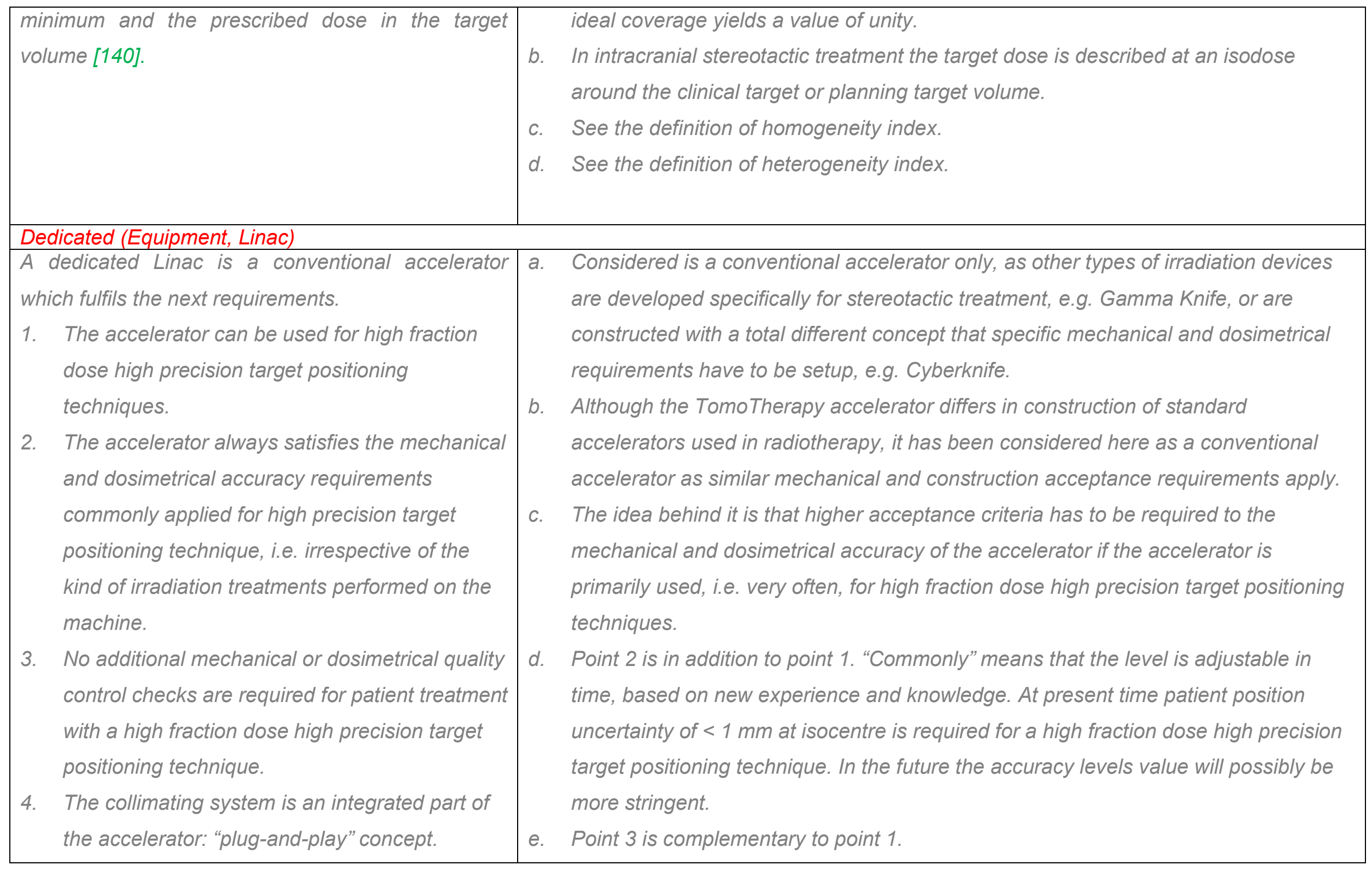




\begin{tabular}{|c|c|}
\hline $\begin{array}{l}\text { The Gamma Knife and Cyberknife are intrinsic } \\
\text { dedicated for stereotactic treatment. }\end{array}$ & $\begin{array}{l}\text { f. Contrary to the "plug-and-play" concept is the "plug-check-and play" concept. Add- } \\
\text { ons like MLC has to be handled according to the latter, requiring a check on correct } \\
\text { performance before use. Cones might fulfil the "plug-and-play" concept as long as } \\
\text { identical accuracy requirements as stated in point } 2 \text { and } 3 \text { can be assured. }\end{array}$ \\
\hline \multicolumn{2}{|l|}{ Fiducial } \\
\hline $\begin{array}{l}\text { A fiducial is an internal landmark directly related to } \\
\text { the target such that it yields information about target } \\
\text { position and preferably target orientation. }\end{array}$ & $\begin{array}{l}\text { a. In general a marker, e.g. golden seed, is implanted in or near the target for optimal } \\
\text { effective determination of target position and orientation. } \\
\text { b. However, a marker or structure might be handled as fiducial if it yields exact } \\
\text { information about target position, and orientation if possible: corresponding to the } \\
\text { intended description of fiducial. } \\
\text { c. For example: the spine for a spine target. } \\
\text { d. See definition of marker. }\end{array}$ \\
\hline \multicolumn{2}{|l|}{ Fused-image set } \\
\hline $\begin{array}{l}\text { A fused-image set is a single patient dataset, } \\
\text { created from other patient image datasets and }\end{array}$ & \\
\hline
\end{tabular}




\begin{tabular}{|c|c|}
\hline \multicolumn{2}{|l|}{ merged and stored as a new image dataset. } \\
\hline \multicolumn{2}{|l|}{ Heterogeneity Index } \\
\hline $\begin{array}{l}\text { The heterogeneity index is defined as the ratio of the } \\
\text { maximum and the prescribed dose in the target } \\
\text { volume. }[14,49,82,163]\end{array}$ & $\begin{array}{l}\text { a. According to this definition a value of } \leq 1.0 \text { means no over-dosage in the target } \\
\text { b. In stereotactic treatment a high heterogeneity in dose distribution over the target } \\
\text { volume is accepted: see table "Treatment Planning, Dose prescription and } \\
\text { Treatment Planning". } \\
\text { c. See the definition of coverage index. } \\
\text { d. See the definition of homogeneity index. }\end{array}$ \\
\hline \multicolumn{2}{|c|}{ High fraction dose high precision target positioning techniques } \\
\hline $\begin{array}{l}\text { A radiation treatment technique in which for each } \\
\text { fraction the highest accuracy in clinical practice can } \\
\text { be achieved in target positioning with respect to the } \\
\text { radiation beams. In addition, in this technique a high } \\
\text { fraction dose is delivered in a limited amount of } \\
\text { fractions. }\end{array}$ & $\begin{array}{l}\text { a. Much more to most conventional radiotherapy treatments, here differences between } \\
\text { the planning target position and the treatment target position with respect to the } \\
\text { radiation beams are minimised in each fraction (on-line correction). } \\
\text { b. High precision in target positioning can be obtained by using fixation or by an image } \\
\text { guided technique. } \\
\text { c. High fraction dose refers to a fraction dose at least twice the fraction dose of a } \\
\text { conventional fractionation schedule. }\end{array}$ \\
\hline \multicolumn{2}{|l|}{ Homogeneity Index } \\
\hline $\begin{array}{l}\text { The homogeneity index is defined as the ratio of the } \\
\text { minimum and maximum dose in the target volume } \\
{[82,117,140,161]}\end{array}$ & $\begin{array}{l}\text { a. According to this definition a value of }<1 \text { means a dose variation over the target. } \\
\text { b. See the definition of coverage index. } \\
\text { c. See the definition of heterogeneity index. }\end{array}$ \\
\hline
\end{tabular}




\begin{tabular}{|c|c|}
\hline $\begin{array}{l}\text { Hyper-fractionation is a dose delivery strategy with } \\
\text { an increased number of fractions compared to } \\
\text { conventional fractionation schedule. Usually, it is } \\
\text { accompanied with a reduced fraction dose to } \\
\text { maintain the same Biological Equivalent Dose (BED) } \\
\text { [e.g. 57] }\end{array}$ & $\begin{array}{l}\text { a. Typical example is the treatment strategy of continuous treatment including week- } \\
\text { end. } \\
\text { b. In general the overall length of treatment time differs from that applied under the } \\
\text { conventional fractionation schedule. } \\
\text { c. See the definition of accelerated fractionation } \\
\text { d. See the definition of hypo-fractionation }\end{array}$ \\
\hline
\end{tabular}




\begin{tabular}{|c|c|}
\hline \multicolumn{2}{|l|}{ See definition of fused-image set. } \\
\hline \multicolumn{2}{|l|}{ Isocentre } \\
\hline $\begin{array}{l}\text { The isocentre is that point in space of the treatment } \\
\text { machine at which the central beam axis of the } \\
\text { radiation beam is always directed, irrespective of the } \\
\text { position in space of the radiation beam focal spot. }\end{array}$ & $\begin{array}{l}\text { a. The central beam axis is the rotational symmetry axis through the focal spot at one } \\
\text { side and the mechanical geometric centre of the beam aperture at the other side of } \\
\text { the radiation delivering part of the treatment machine. } \\
\text { b. For linear accelerators our definition of the isocentre corresponds with the original } \\
\text { isocentre definition [77], i.e. the cross point of the mechanical axis of treatment head } \\
\text { en gantry. } \\
\text { c. For TomoTherapy devices our definition yields the mechanical geometrical centre } \\
\text { d. Foint of the ring on which the radiation tube rotates. } \\
\text { sphere on which the irradiation sources are distributed. } \\
\text { e. For Cyberknife systems our isocentre point cannot been deduced from mechanical } \\
\text { characteristics of the machine. } \\
\text { f. Here, the calibration procedure of the Cyberknife system yields a point satisfying our } \\
\text { definition of isocentre. See Glossary. }\end{array}$ \\
\hline \multicolumn{2}{|l|}{ Manufacturer } \\
\hline $\begin{array}{l}\text { The company who produces equipment and is liable } \\
\text { for its functioning as total system, conform European } \\
\text { and / or national law. } \\
\text { See too: Vendor }\end{array}$ & $\begin{array}{l}\text { a. The manufacturer has to certify his equipment according to CE-marking (Conformité } \\
\text { Européenne), required by European Directive. } \\
\text { b. In selling equipment to customers, the manufacturer might be represented by a } \\
\text { vendor which is not commercial unit of that manufacturer. } \\
\text { c. Often a manufacturer offers a package of own-produced equipment. In general, that }\end{array}$ \\
\hline
\end{tabular}


d. An example is the Gamma Knife and Cyberknife system.

\section{Marker}

A marker is an external landmark with the aim to

a.

a. In general there is a relationship assumed between marker and target, although

identify the position, and if possible, the orientation often not that exact as there is between a fiducial and target.

of the target.

b. See definition of fiducial.

\section{Master-image set}

A master-image set is a patient image dataset used

as base for treatment planning, co-registration and

treatment delivery.

\section{Non-dedicated treatment equipment}

Non-dedicated treatment equipment is equipment

that does not fulfil the requirements for as much as

inherent safe stereotactic treatments.

a. In non-dedicated equipment, there are aspects influencing the treatment safety and quality in a negative way.

b. For instance: they may yield additional risk of process or technical errors.

c. Think about: add-ons, self-written software, work-around needed, not fully compatible appliance, etc.

\section{Overlaid-image dataset}

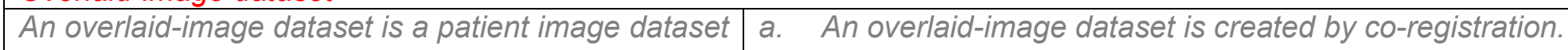

on which other patient image datasets are overlaid.

b. An overlaid image dataset is not stored as a new patient image dataset.

c. Each of the underlying patient image datasets remains an independent dataset. 


\begin{tabular}{|c|c|}
\hline & $\begin{array}{l}\text { d. See definition of registration. } \\
\text { e. See definition of fused-image set. }\end{array}$ \\
\hline $\begin{array}{l}\text { Plan Do Check Act cycle is an iterative four-step } \\
\text { management method used in business for the } \\
\text { control and continuous improvement of processes } \\
\text { and products. }\end{array}$ & $\begin{array}{l}\text { a. Plan: set- up a plan, based on a strategy and mission of the department, in which } \\
\text { success-factors, norm-points and their critical points to realize them have been } \\
\text { identified. } \\
\text { b. Do: perform and manage the plan. } \\
\text { c. Check: compare plan-performs against success-factor, norm-points and their critical } \\
\text { points. } \\
\text { d. Act: adjust process if needed. } \\
\text { e. Most critical points: 1) no reflection on plan and performance (no realistic check and } \\
\text { act moment), 2) no try and test moment,3) PDCA is only part of a process analysis to } \\
\text { repair, 4) no real intention to evaluate, 5) no integrated action between management } \\
\text { and performers. }\end{array}$ \\
\hline \multicolumn{2}{|l|}{ Reference frame } \\
\hline $\begin{array}{l}\text { A reference frame is a rigid structure allowing an } \\
\text { unambiguous transformation of patient (target / } \\
\text { organs at risk) position coordinates from one space } \\
\text { to another. }\end{array}$ & $\begin{array}{l}\text { a. A reference frame can be external, e.g. a rigid metal structure fixed to the skull like } \\
\text { the invasive frame, or internal, e.g. the skull, a vertebra or a fiducial: as long as the } \\
\text { target / organs at risk is / are in unambiguous position relative to this structure. } \\
\text { b. A reference frame is assumed representative of the patient anatomy, i.e. } \\
\text { transportation of the patient anatomy from one space to another is unambiguously } \\
\text { related to a similar transportation of the frame from one coordinate system to } \\
\text { another. }\end{array}$ \\
\hline
\end{tabular}




\begin{tabular}{|c|c|c|}
\hline & $\begin{array}{l}\text { c. } \\
\text { d. } \\
\text { e. } \\
\text { f. } \\
\text { g. }\end{array}$ & $\begin{array}{l}\text { Frames not transforming unambiguously the patient position coordinates from one } \\
\text { space to another cannot be considered as reference frame according to the } \\
\text { definition in this report. } \\
\text { Such frames might only support a good immobilisation of the patient. } \\
\text { A "frameless stereotactic treatment technique" means that no invasive or relocatable } \\
\text { frame has been applied to transport the target / organ at risk position coordinates to } \\
\text { the treatment coordinates system. An internal frame is used for that, see note a) } \\
\text { above. } \\
\text { See frameless system in the Glossary. } \\
\text { See definition of fixation. }\end{array}$ \\
\hline \multicolumn{3}{|l|}{ Registration } \\
\hline $\begin{array}{l}\text { Registration is a method to transform one patient } \\
\text { dataset to another with the aim to identify identical } \\
\text { points in both datasets. }[25,45,68,71,113,125 \text {, } \\
\text { 147] }\end{array}$ & a. & $\begin{array}{l}\text { The transformation might be rigid or non-rigid, i.e. deformed in that transformation } \\
\text { procedure. }\end{array}$ \\
\hline \multicolumn{3}{|l|}{ Stereotactic Radiosurgery (SRS) } \\
\hline \multicolumn{3}{|l|}{$\begin{array}{l}\text { Stereotactic Radiosurgery is a stereotactic treatment } \\
\text { in where the total dose is delivered in a single } \\
\text { session. [93] }\end{array}$} \\
\hline \multicolumn{3}{|l|}{ Stereotactic treatment } \\
\hline In this report we describe stereotactic treatment as a & a. & The condition of "reference frame" is related to the requirement of a non-ambiguous \\
\hline
\end{tabular}




\begin{tabular}{|c|c|c|}
\hline $\begin{array}{l}\text { high fraction dose high precision target positioning } \\
\text { technique that rely on the use of a reference frame. }\end{array}$ & & $\begin{array}{l}\text { transformation of target and organs at risk coordinates from one coordinates system } \\
\text { to another. }\end{array}$ \\
\hline $\begin{array}{l}\text { Targeting error is the offset of the delivered dose } \\
\text { distribution to the intended dose distribution to a } \\
\text { (spherical) target positioned in a (cubical) phantom. }\end{array}$ & a. & $\begin{array}{l}\text { Mechanical and image-guided tools can be applied to position the spherical target } \\
\text { with highest obtainable accuracy at the isocentre. }\end{array}$ \\
\hline \multicolumn{3}{|l|}{ Tolerance level } \\
\hline $\begin{array}{l}\text { The tolerance level is defined as that accuracy limit } \\
\text { that is accepted as allowed deviation between the } \\
\text { actual status of a parameter and its ideal status to } \\
\text { use the system in a safe and proper way for clinical } \\
\text { patient treatment. }\end{array}$ & a. & $\begin{array}{l}\text { If the deviation between the actual status of a parameter and its ideal status is below } \\
\text { or equal to the tolerance level, no further action is required. } \\
\text { If the deviation between the actual status of a parameter and its ideal status is } \\
\text { between the tolerance and action level, several courses of action are open. } \\
\text { For a problem that is easily and quickly rectifiable, remedial action should be taken } \\
\text { at once. } \\
\text { A first alternative response is to delay remedial intervention until the next scheduled } \\
\text { maintenance period. } \\
\text { A second alternative response is to decide to monitor the performance of the } \\
\text { parameter in question over a period of time and to postpone a decision until the } \\
\text { behaviour of the parameter is confirmed. } \\
\text { However, the way of response is always to guarantee a safe and proper way for } \\
\text { clinical patient treatment and based on a decision made by the supervising experts } \\
\text { in consultation with the users of the equipment and others as appropriate. }\end{array}$ \\
\hline
\end{tabular}




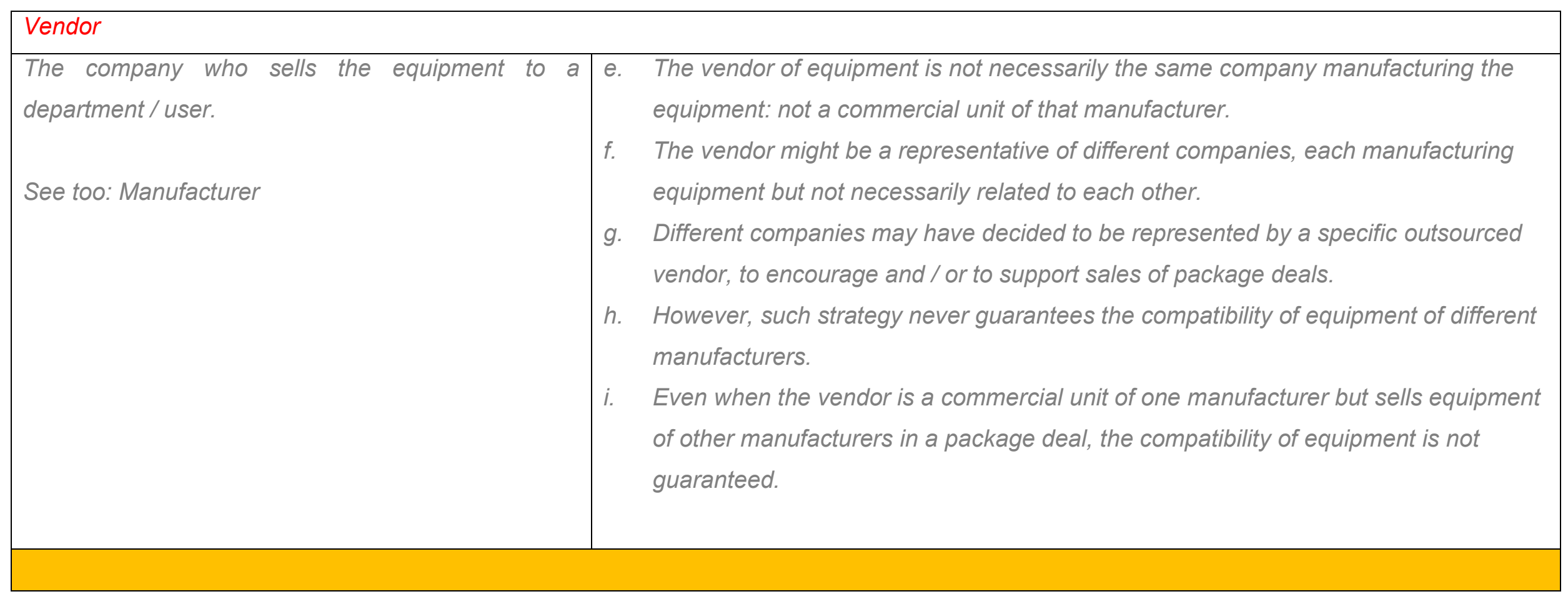

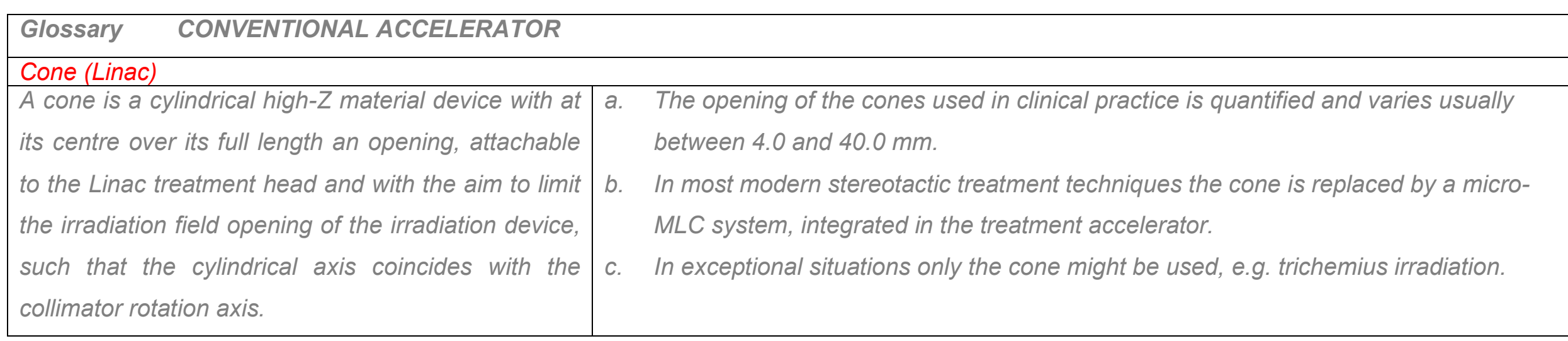




\begin{tabular}{|c|c|}
\hline \multicolumn{2}{|l|}{ ExacTrac System } \\
\hline $\begin{array}{l}\text { ExacTrac system is a patient alignment system, } \\
\text { forcing robotic treatment couch movements. }\end{array}$ & $\begin{array}{l}\text { a. ExacTrac system consists of: } \\
\text { - Infra-red system, detecting markers and used for a rough target set-up at } \\
\text { isocentre. } \\
\text { - X-ray system to detected fiducials or bony structures, used for fine-adjustment } \\
\text { of target set-up at isocentre. } \\
\text { Interface to the robotic treatment couch to force its movements to fine-align } \\
\text { target set-up at isocentre. } \\
\text { b. It is strongly advised to test the movement of the robotic treatment couch induced by } \\
\text { the ExacTrac system when both originate from different manufacturers. } \\
\text { c. Calibration procedure is described in the ExacTrac software itself. } \\
\text { d. Quality control of the calibration procedure is based on an independent X-ray based, } \\
\text { checking the isocentre projection by applying the Winston Lutz test tool with on the } \\
\text { X-ray imagers. }\end{array}$ \\
\hline \multicolumn{2}{|l|}{ Frameless (System) } \\
\hline $\begin{array}{l}\text { A Frameless System is a combined strong } \\
\text { immobilisation and marker system with the explicit } \\
\text { ambition to be handled as a non-invasive reference } \\
\text { frame. }\end{array}$ & $\begin{array}{l}\text { a. The ambition requires that a Frameless System can be handled as a reference } \\
\text { frame (see table "definitions"). The term "frameless" reflects the ambition to avoid } \\
\text { using an invasive frame. } \\
\text { b. To fulfil the ambition, a Frameless System can be handled as a reference frame. } \\
\text { Two potential weak links of the system should be investigated by the user: } \\
\text { - A Frameless System is based on immobilisation (see table "definitions") } \\
\text { principles instead of fixation (see table "definitions") }\end{array}$ \\
\hline
\end{tabular}




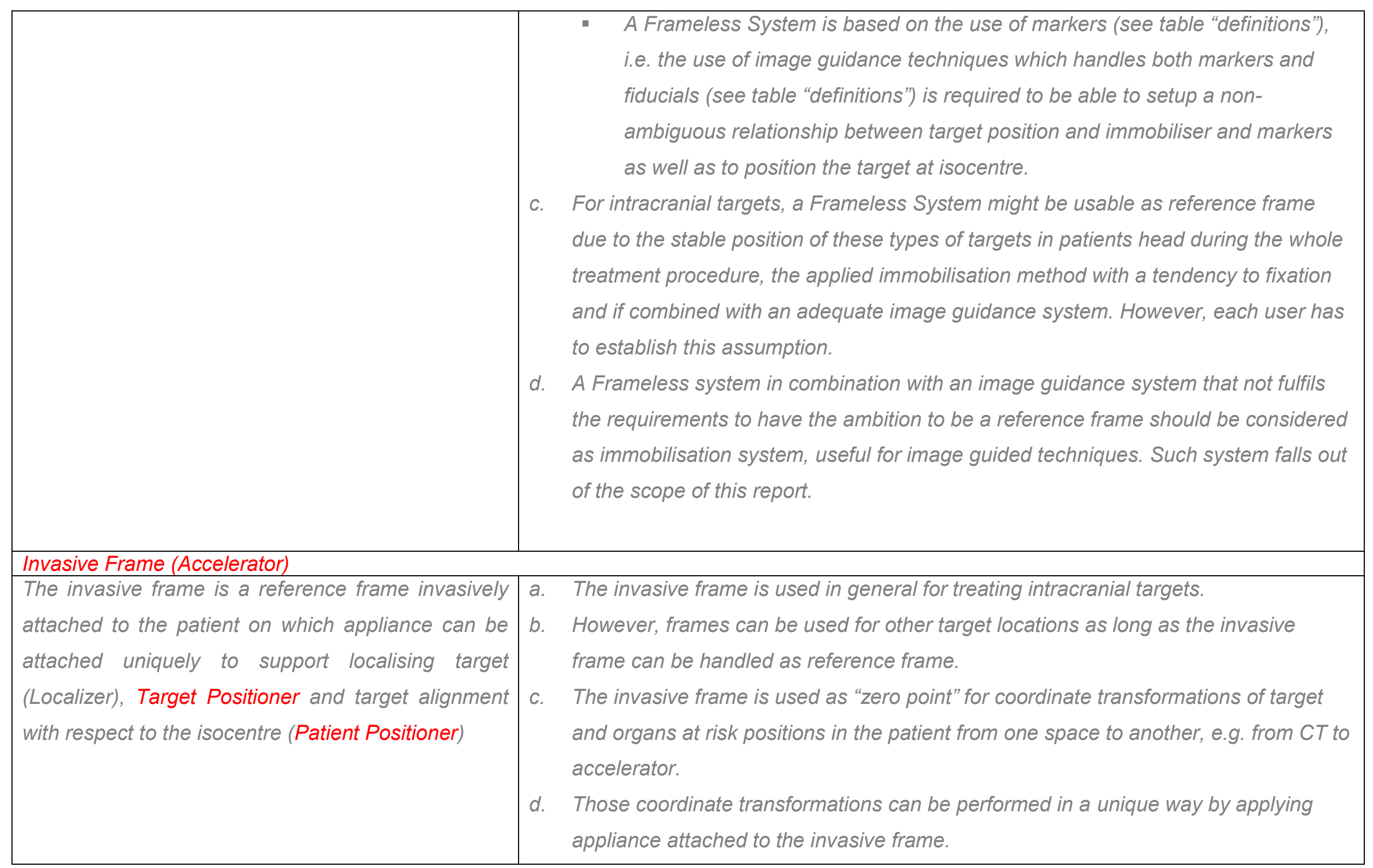




\begin{tabular}{|c|c|}
\hline & $\begin{array}{l}\text { e. In general it is not possible to use a mix of invasive frame and appliance from } \\
\text { different manufacturers, including home-made appliance. } \\
\text { f. However, it should be realised that if a mixed use is possible, it is dangerous to do } \\
\text { so as each manufacturer constructs invasive frame and appliance as a unique } \\
\text { combination. } \\
\text { g. In addition, treatment planning software from or advised by the manufacturer of the } \\
\text { invasive frame and its belonging appliance is strongly advised to use to handle those } \\
\text { devices correctly and safely in dose treatment planning and dose delivery. } \\
\text { h. If treatment planning software is used other than advised by the manufacturer of the } \\
\text { invasive frame and belonging appliance, it should be realised that a very careful and } \\
\text { extended test is required of proper functioning of that planning software with respect } \\
\text { to the applied devices. } \\
\text { An invasive frame used for intracranial targets is attached tight to the (top of each) } \\
\text { couch to avoid any movement of the patient during irradiation. } \\
\text { Small translation and rotations, i.e. several cm and degrees, respectively, of the } \\
\text { frame can be setup with respect to that couch, to allow high accurate alignment of } \\
\text { target and target positioner at isocentre position. }\end{array}$ \\
\hline \multicolumn{2}{|l|}{ Localizer } \\
\hline $\begin{array}{l}\text { The Localizer is a high-accurate hardware system } \\
\text { attachable to the invasive frame which the aim to } \\
\text { localize in a unique way target and organs at risk } \\
\text { position in the patient relative to the invasive frame. }\end{array}$ & $\begin{array}{l}\text { a. A localizer is used, for instance, during CT or MRI scanning of a patient. } \\
\text { b. Treatment planning software which allow apply of invasive frame and its belonging } \\
\text { appliance for stereotactic treatment, requires a facility to recognize such localizers } \\
\text { automatically and to use those localizers as coordinate transfer system for target } \\
\text { and organs at risk position from one space to another. }\end{array}$ \\
\hline
\end{tabular}




\begin{tabular}{|c|c|}
\hline \multicolumn{2}{|l|}{ Patient Positioner } \\
\hline $\begin{array}{l}\text { Patient Positioner is a high-accurate constructed } \\
\text { system attachable to the invasive frame, positioned } \\
\text { over the patient where the target is located, with the } \\
\text { aim for accurate positioning of the target with } \\
\text { respect to the isocentre. }\end{array}$ & $\begin{array}{l}\text { a. Treatment planning software which allows apply of invasive frame and its belonging } \\
\text { appliance for stereotactic treatment, requires a facility to project with high accuracy } \\
\text { target position in the patient on the Patient Positioner. } \\
\text { b. For irradiation of targets in patients head the Patient Positioner consists of a four- } \\
\text { wall box. } \\
\text { c. With this target projections on the Patient Positioner target in the patient can be } \\
\text { aligned on the treatment room laser system with high accuracy. }\end{array}$ \\
\hline \multicolumn{2}{|l|}{ Pitch } \\
\hline \multicolumn{2}{|l|}{$\begin{array}{l}\text { The pitch is the rotation angle of the treatment couch } \\
\text { over its longitudinal axis. }\end{array}$} \\
\hline
\end{tabular}




\begin{tabular}{|c|c|}
\hline & $\begin{array}{l}\text { is for the invasive frame. } \\
\text { f. If the relocatable frame system is obtained from the same manufacturer delivering } \\
\text { the invasive system to be replaced, the appliance belong to that invasive frame can } \\
\text { be used in general too in combination with the relocatable system. } \\
\text { g. Part of the relocatable frame is a control-helmet attachable to the relocatable frame } \\
\text { and positioned over the patients head. } \\
\text { h. The aim of this control-helmet is to measure at a large number of points the distance } \\
\text { between helmet and head of the patient each time the relocatable frame is } \\
\text { repositioned. }\end{array}$ \\
\hline $\begin{array}{l}\text { Robotic treatment couch } \\
\text { Robotic treatment couch is a treatment couch } \\
\text { allowing accurate rotations over longitudinal and } \\
\text { lateral couch axis, i.e. pitch and roll, respectively, } \\
\text { beside the standard allowed movements of similar } \\
\text { conventional treatment couches. }\end{array}$ & \\
\hline $\begin{array}{l}\text { Roll } \\
\text { The roll is the rotation angle of the treatment couch } \\
\text { over its lateral axis. }\end{array}$ & \\
\hline $\begin{array}{l}\text { Target Positioner } \\
\text { The Target Positioner is a high-accurate expedient } \\
\text { attachable to the invasive frame, containing a high-Z }\end{array}$ & $\begin{array}{l}\text { A. A target positioner is used on the accelerator for radiation simulation of the intended } \\
\text { patient treatment technique. }\end{array}$ \\
\hline
\end{tabular}




\begin{tabular}{|c|c|c|}
\hline $\begin{array}{l}\text { material sphere (target, } \varnothing=2-3 \mathrm{~mm} \text { ) adjustable in } \\
\text { position to that invasive frame, with the aim to } \\
\text { represent target position, i.e. high-Z material sphere, } \\
\text { relative to the invasive frame. }\end{array}$ & $b$. & $\begin{array}{l}\text { Treatment planning software which allows applies of invasive frame and its } \\
\text { belonging appliance for stereotactic treatment, requires a facility to calculate target } \\
\text { position with respect to the invasive frame. }\end{array}$ \\
\hline \multirow[b]{2}{*}{$\begin{array}{l}\text { Winston-Lutz test } \\
\text { The Winston-Lutz test is a mechanical quality control } \\
\text { (QC) procedure on a conventional accelerator as } \\
\text { suggested by Winston and Lutz. The aim is to } \\
\text { control the mutual alignment of the treatment room } \\
\text { laser system, couch rotation axis and mechanical } \\
\text { and X-ray beam isocentre of that accelerator for } \\
\text { gantry and couch angles in all possible } \\
\text { combinations. [132] }\end{array}$} & & \\
\hline & $d$. & $\begin{array}{l}\text { The Winston Lutz QC tool consists of a high-Z material sphere ( } \varnothing=2-3 \mathrm{~mm} \text { ) } \\
\text { enclosed in a small Perspex cube. } \\
\text { Each plane of the cube at contains an orthogonal cross-line with its centre at the } \\
\text { centre of the plane. } \\
\text { The Winston Lutz QC tool is fixed on the top of the treatment couch and positioned } \\
\text { at the mechanical isocentre applying the cross-lines and the treatment room laser } \\
\text { system. } \\
\text { X-ray images of the sphere are generated for several combinations of gantry and } \\
\text { couch angle. } \\
\text { To investigate the sphere position with respect to the X-ray isocentre, the X-ray } \\
\text { beam used is collimated by a cylindrical high-Z material cone with an opening of } \\
\text { about } 2 \text { cm, fixed on the treatment head and aligned exactly on the collimator } \\
\text { rotation axis using a dial indicator. } \\
\text { On the X-ray images the position of the sphere is analysed with respect to the } \\
\text { cylindrical opening. From this information the mutual position of the treatment laser } \\
\text { system, couch rotation axis and mechanical and X-ray isocentre can be deduced. }\end{array}$ \\
\hline
\end{tabular}




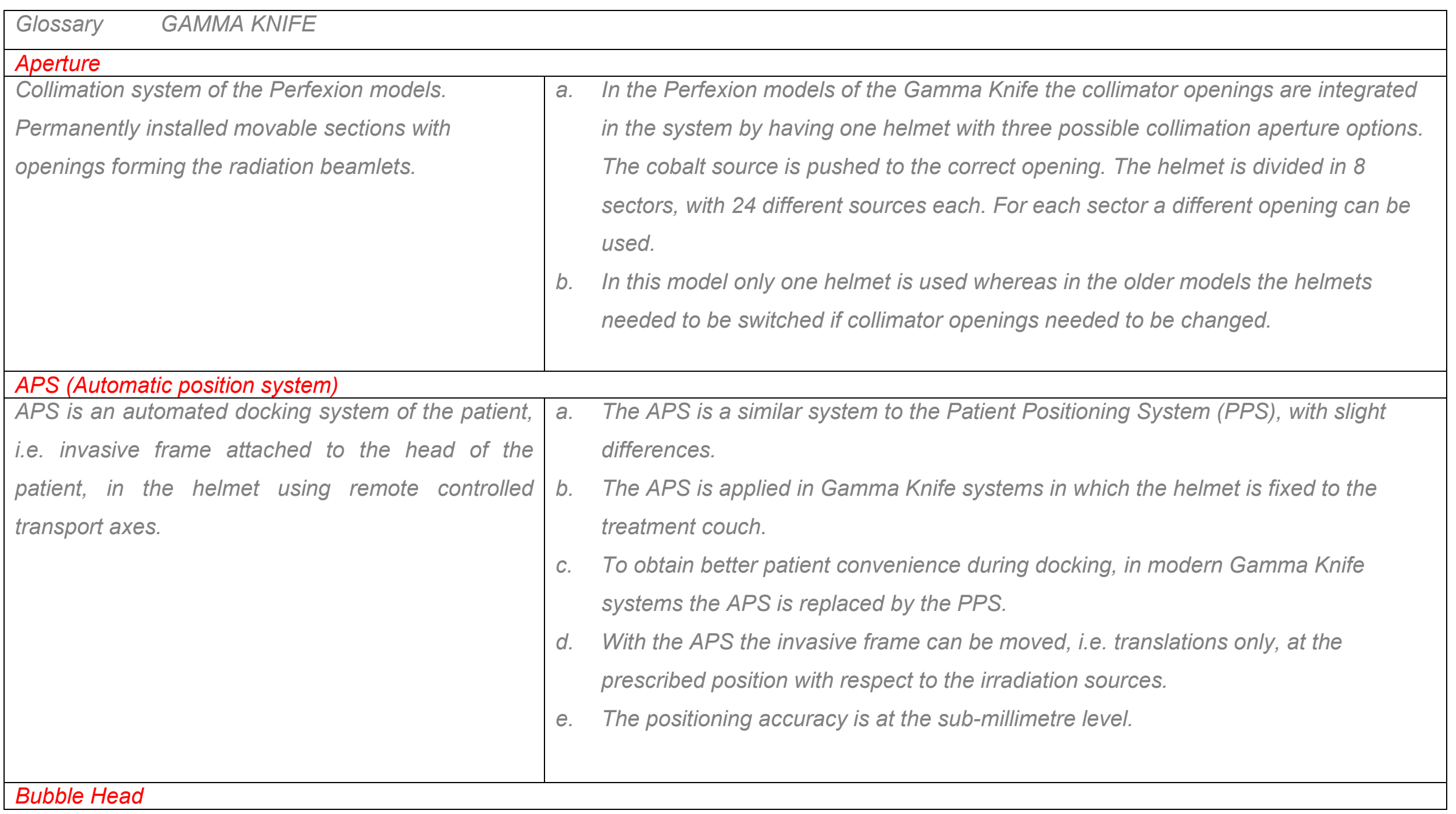




\begin{tabular}{|c|c|}
\hline $\begin{array}{l}\text { The bubble head is a hemi-spherical bowl which can } \\
\text { be positioned over the head of a patient and can be } \\
\text { fixed in a unique way to the invasive frame. }\end{array}$ & $\begin{array}{l}\text { a. The aim of the bubble head is to measure at a large number of points the distance of } \\
\text { the head of the patient to the bubble head. } \\
\text { b. With that information the head of the patient is modelled in the treatment planning } \\
\text { software for dose calculation reasons. }\end{array}$ \\
\hline \multicolumn{2}{|l|}{ Collimator (Gamma Knife) } \\
\hline $\begin{array}{l}\text { A collimator is a cylindrical high-Z material device } \\
\text { with at its centre over its full length an opening. It is } \\
\text { attachable in the helmet with the aim to limit the } \\
\text { irradiation field opening of a treatment source. }\end{array}$ & $\begin{array}{l}\text { a. A model C Gamma Knife helmet has } 201 \text { openings for collimators. } \\
\text { b. The central opening of the collimators used in clinical practice has discrete values } \\
\text { varies between } 0 \text { (i.e. closed) and } 4 \text { to } 18 \mathrm{~mm} \text {. } \\
\text { c. In modern Gamma Knife systems the set of cones is replaced by a computer } \\
\text { controlled aperture system, integrated in the helmet inside the Gamma Knife. } \\
\text { d. A collimator with } 0 \mathrm{~mm} \text { opening is called a plug. }\end{array}$ \\
\hline
\end{tabular}




\begin{tabular}{|c|c|}
\hline $\begin{array}{l}\text { The helmet is a hemi-spherical irradiation source } \\
\text { collimator system. }\end{array}$ & $\begin{array}{l}\text { a. The aim of the helmet is allow collimation of the irradiation sources of the Gamma } \\
\text { b. Thife system such that a proper treatment of the target can be applied. } \\
\text { values, applying a computer controlled aperture system per hole integrated in the } \\
\text { helmet or applying cones which can be plugged manually into the holes. } \\
\text { c. Depending on the Gamma Knife system used, the helmet can be docked in a unique } \\
\text { way to the irradiation sources (no specific name for that docking software) or the } \\
\text { helmet is already fixed to these irradiation sources. } \\
\text { d. In the first case, the helmet is fixed to the treatment couch while APS is used as } \\
\text { docking system of the patient to the helmet. } \\
\text { e. In the second case the whole treatment couch is docked to the helmet. }\end{array}$ \\
\hline \multicolumn{2}{|l|}{ Invasive Frame (Gamma Knife) } \\
\hline $\begin{array}{l}\text { The invasive frame-Gamma Knife is a reference } \\
\text { frame invasive attached to the patient and on which } \\
\text { appliance can be attached uniquely to support } \\
\text { localising target and target alignment with respect to }\end{array}$ & $\begin{array}{l}\text { a. The invasive frame for Gamma Knife treatments is used for treating targets in } \\
\text { patients head. } \\
\text { b. The invasive frame is used as "zero point" for coordinate transformations of target } \\
\text { and organs at risk positions in the patient from one space to another. }\end{array}$ \\
\hline
\end{tabular}




\begin{tabular}{|c|c|}
\hline the irradiation sources. & $\begin{array}{l}\text { c. Those coordinate transformations can be performed in a unique way by applying } \\
\text { appliance attached to the invasive frame. } \\
\text { d. An invasive frame used for targets located in patients head is attached tight to the } \\
\text { (top of each) couch or APS to avoid any movement of the patient during irradiation. } \\
\text { e. Translation and rotations of the frame can be setup with respect to the sources, to } \\
\text { allow high accurate alignment of target and target positioner at isocentre position. } \\
\text { f. See for example gamma angle. }\end{array}$ \\
\hline \multicolumn{2}{|l|}{ PPS (Patient Positioning System) } \\
\hline $\begin{array}{l}\text { Patient Positioning System is an automated docking } \\
\text { system of the patient, i.e. invasive frame attached to } \\
\text { the head of the patient, in the helmet using remote } \\
\text { controlled transport axes. }\end{array}$ & $\begin{array}{l}\text { a. The PPS is similar system to the Automatic Positioning System (APS), with slight } \\
\text { differences. } \\
\text { b. The PPS is applied in Gamma Knife systems in which the helmet is fixed to the } \\
\text { irradiation sources. } \\
\text { c. With the PPS the treatment couch can be moved, i.e. translations only, at the } \\
\text { prescribed position with respect to the irradiation sources. } \\
\text { d. The positioning accuracy is at the sub-millimetre level. } \\
\text { e. The PPS is docked into the helmet for patient source alignment. } \\
\text { f. Therefore, PPS replaced APS in modern Gamma Knife systems. }\end{array}$ \\
\hline \multicolumn{2}{|l|}{ Trunnion } \\
\hline $\begin{array}{l}\text { Trunnion is a manual docking system of the patient, } \\
\text { i.e. invasive frame attached to the head of the } \\
\text { patient, in the helmet. In other words: it is the } \\
\text { manual executable of the APS or PPS. }\end{array}$ & \\
\hline
\end{tabular}




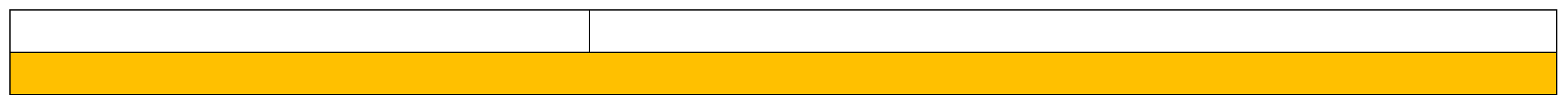

\begin{tabular}{|c|c|}
\hline CYBERKNIFE & \\
\hline Collimator Systems (Cyberknife) & \\
\hline $\begin{array}{l}\text { 1. Fixed collimators (Cones) } \\
\text { The Cyberknife has two collimators, a fixed primary } \\
\text { collimator and a changeable secondary collimator. } \\
\text { The primary collimator surrounds the X-ray target } \\
\text { and limits the X-ray beam to a narrow cone in the } \\
\text { forward direction. Secondary collimators may be } \\
\text { attached to the X-ray head to produce different } \\
\text { beam sizes. }\end{array}$ & $\begin{array}{l}\text { a. The Cyberknife has } 12 \text { interchangeable fixed collimators with apertures of 5, 7.5, } 10 \text {, } \\
12.5,15,20,25,30,35,40,50,60 \mathrm{~mm} \text {, defined at a distance of } 80 \mathrm{~cm} \text { from the } X \text { - } \\
\text { ray source. }\end{array}$ \\
\hline 2. Incise Multileaf Collimator (MLC) & $\begin{array}{l}\text { a. The optional Incise Multileaf Collimator (MLC) is a secondary collimator whose } \\
\text { aperture is adjustable under computer control. } \\
\text { b. Using tungsten leaves to rapidly adjust the aperture, the MLC can deliver variable } \\
\text { shaped beams from each treatment head position. }\end{array}$ \\
\hline 3. Iris Variable Aperture Collimator & $\begin{array}{l}\text { a. The optional Iris Variable Aperture Collimator provides the same } 12 \text { apertures as the } \\
\text { fixed collimators. } \\
\text { b. The aperture of the Iris Collimator is adjustable under computer control. }\end{array}$ \\
\hline
\end{tabular}




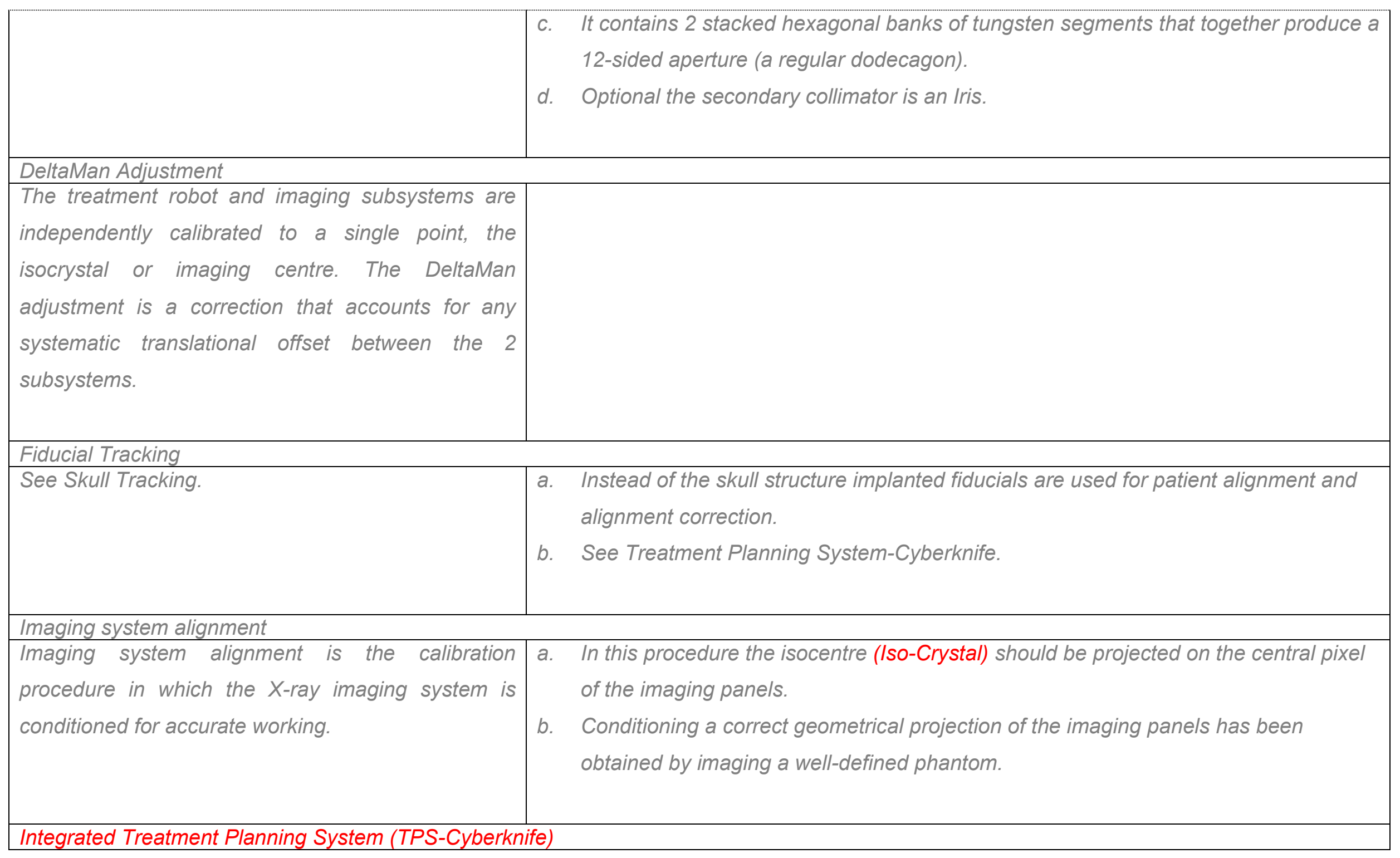




\begin{tabular}{|c|c|}
\hline $\begin{array}{l}\text { TPS-Cyberknife is the build-in treatment planning } \\
\text { software in the Cyberknife system to guarantee by } \\
\text { the manufacturer a non-ambiguous interface to force } \\
\text { the treatment delivery as intended by dose } \\
\text { calculation. }\end{array}$ & $\begin{array}{l}\text { a. TPS-Cyberknife is working too as a record and verify system of the Cyberknife } \\
\text { hardware. } \\
\text { b. It is dangerous to use another software packet as supplied by the manufacturer of } \\
\text { the Cyberknife system as the interface between software (dose prescription) and } \\
\text { hardware is not optimal established as can be expected and required from the } \\
\text { manufacturer of the Cyberknife. } \\
\text { c. Reason for this is that another software designer as the manufacturer is in general } \\
\text { not optimal informed about all technical aspects of the hardware to be able to } \\
\text { guarantee an optimal functioning of its software. } \\
\text { d. Software designed for optimal patient (target) positioning with respect to the } \\
\text { irradiation beam should be considered as an integrated part of the treatment } \\
\text { planning software due to the specific interface with the Cyberknife hardware. } \\
\text { e. TPS software packets delivered by the manufacturer may have names different from } \\
\text { "Cyberknife". }\end{array}$ \\
\hline \multicolumn{2}{|l|}{ Iso-Crystal / Iso-Crystal p } \\
\hline $\begin{array}{l}\text { The Iso-Crystal is a 3D isotropic light detector, i.e. a } \\
\text { white sphere of about } 3 \mathrm{~mm} \text { diameter, at the tip of a } \\
\text { post, used as a tool to represent the isocentre of the } \\
\text { Cyberknife. }\end{array}$ & $\begin{array}{l}\text { a. The isocentre of the Cyberknife cannot be defined like for conventional linear } \\
\text { accelerators. } \\
\text { b. To ensure that the radiation beam of the Cyberknife can be directed to a single point } \\
\text { in space, an isocentre is defined by the manufacturer applying the Iso-Crystal. } \\
\text { c. During calibration and quality control of the Cyberknife system the Iso-Crystal post is } \\
\text { mounted in the floor frame of the Cyberknife between the imager panels in a unique } \\
\text { and reproducible way. } \\
\text { d. The 3D isotropic light detector and the software behind allow determination of the }\end{array}$ \\
\hline
\end{tabular}




\begin{tabular}{|c|c|}
\hline & $\begin{array}{l}\text { position and direction of the laser beam in space. } \\
\text { e. Irradiating the detector from a particular direction, it owns the capability to detect } \\
\text { light intensity variation when varies slightly that direction. } \\
\text { f. This capability is used to register robotic arms position (mechanical alignment). }\end{array}$ \\
\hline \multicolumn{2}{|l|}{ Laser Cyberknife } \\
\hline $\begin{array}{l}\text { The Cyberknife System uses a pinhole laser as a } \\
\text { QA tool; it is positioned in the treatment head and } \\
\text { coincides with the radiation field central axis (CAX). }\end{array}$ & $\begin{array}{l}\text { The laser is used to perform, e.g.: } \\
\text { a. configuration checks on the collimator exchange table; } \\
\text { b. path calibration and verification using an Iso-Crystal. }\end{array}$ \\
\hline \multicolumn{2}{|l|}{ Mechanical alignment of a Cyberknife } \\
\hline $\begin{array}{l}\text { Cyberknife mechanical alignment is a calibration } \\
\text { procedure in two steps in which the movements of } \\
\text { the robotic arms are calibrated such that the } \\
\text { irradiation beam of the Cyberknife is always directed } \\
\text { to the isocentre of the system (Iso-Crystal), } \\
\text { irrespective of the position of the beam focus in } \\
\text { space. }\end{array}$ & $\begin{array}{l}\text { a. The first step in this procedure is the first-order calibration of the movements of the } \\
\text { robotic arms by applying the Cyberknife laser (Robot mastering) } \\
\text { b. The second step is based on X-ray imaging of the isocentre, the X-ray beam coming } \\
\text { from different directions, yielding a correction on the calibration results obtained by } \\
\text { the Cyberknife laser system. } \\
\text { c. Essential in this procedure is a correct alignment of the imaging system (Imaging } \\
\text { system alignment) }\end{array}$ \\
\hline \multicolumn{2}{|l|}{ Node } \\
\hline $\begin{array}{l}\text { A node is a stopping point in } 3 D \text { space of the } \\
\text { irradiation beam focus with the irradiation beam } \\
\text { directed towards the target. }\end{array}$ & $\begin{array}{l}\text { a. For security reasons the positions of nodes in space in clinical practice is restricted } \\
\text { along paths. } \\
\text { b. At each node a variable amount of different beam directions }(N) \text { can be setup to } \\
\text { irradiate the target. }\end{array}$ \\
\hline
\end{tabular}




\begin{tabular}{|c|c|}
\hline & $\begin{array}{l}\text { c. In clinical practice a large amount of nodes (M) are possible, distributed over a } \\
\text { limited number of paths. } \\
\text { d. Regarding point b) and c) approximately } N^{*} M \text { beam directions are possible. }\end{array}$ \\
\hline \multicolumn{2}{|l|}{ Path way } \\
\hline $\begin{array}{l}\text { A path is a trajectory in } 3 D \text { space to allow the robotic } \\
\text { arm of the Cyberknife to move safely and } \\
\text { consistently. As a consequence a path yields the } \\
\text { trajectory in which the radiation beam focus can be } \\
\text { moved and set (see "node") }\end{array}$ & $\begin{array}{l}\text { a. In principle a trajectory is that part of the 3D space in which the robotic arm can be } \\
\text { moved safely and consistently, i.e. a knock-on process with patient or Cyberknife } \\
\text { b. In clinical practice such trajectories are more restricted to "free-lines" in space to } \\
\text { assure a better consistent behaviour of the Cyberknife. } \\
\text { c. Paths are defined by the manufacturer before acceptance. } \\
\text { d. Along each path a series of nodes can be set on. } \\
\text { e. In most clinical situations } 3 \text { paths are used, related to brain, prostate and lung target } \\
\text { irradiation. }\end{array}$ \\
\hline $\begin{array}{l}\text { A form fitting "highly" elastic vest for patients treated } \\
\text { with help of Synchrony. }\end{array}$ & a. The (disposable) vest is for convenient attachment of the LED tracking markers. \\
\hline \multicolumn{2}{|l|}{ Robot calibration check } \\
\hline $\begin{array}{l}\text { Robotic calibration check of the Cyberknife system } \\
\text { is the procedure to check a correct alignment of the } \\
\text { radiation beam to the isocentre during treatment. }\end{array}$ & $\begin{array}{l}\text { a. In this procedure the Cyberknife internal laser beam irradiates the Iso-Crystal when } \\
\text { the robot passes through nodes distributed over several but a limited number of } \\
\text { paths. } \\
\text { b. The calibration (check) software records the laser readings of the Iso-Crystal and }\end{array}$ \\
\hline
\end{tabular}




\begin{tabular}{|c|c|}
\hline & $\begin{array}{l}\text { compares those actual readings with the laser readings obtained during robot } \\
\text { mastering. } \\
\text { c. In situations of inconsistency in this comparison the causes behind can be different } \\
\text { and should be investigated therefore carefully. }\end{array}$ \\
\hline \multicolumn{2}{|l|}{ Robot mastering } \\
\hline \multicolumn{2}{|l|}{$\begin{array}{l}\text { Robot mastering is the gathering of data from robotic } \\
\text { arms positions obtained in the first step of the } \\
\text { mechanical alignment procedure. }\end{array}$} \\
\hline \multicolumn{2}{|l|}{ Skull Tracking } \\
\hline $\begin{array}{l}\text { Skull tracking is part of the Cyberknife software in } \\
\text { which automatically real-time patient alignment and } \\
\text { alignment correction can be performed during } \\
\text { Cyberknife image-guided intracranial radiotherapy. }\end{array}$ & $\begin{array}{l}\text { a. Applying this tool digitally reconstructed radiographs (DRRs) are generated off-line } \\
\text { from patient CT data before treatment. } \\
\text { b. The DRRs are used as reference images for patient alignment. } \\
\text { c. For patient alignment two orthogonal projected X-ray images are acquired before } \\
\text { treatment to verify and correct patient alignment. } \\
\text { d. Calculated position deviations are used for automatic couch reposition (during } \\
\text { patient set-up) or automatic robotic arm position correction during irradiation. }\end{array}$ \\
\hline \multicolumn{2}{|l|}{ Synchrony® } \\
\hline $\begin{array}{l}\text { Synchrony is a respiratory tracking system, attached } \\
\text { to the Cyberknife system. }\end{array}$ & $\begin{array}{l}\text { a. The system uses infra-red LED breathing motion tracking markers attached to the } \\
\text { patient chest, ideally on a tight Patient Vest, or on treatment couch. } \\
\text { b. The software relates real-time the infra-red marker positions to X-ray obtained } \\
\text { fiducial information. }\end{array}$ \\
\hline
\end{tabular}




\begin{tabular}{|c|c|c|}
\hline & C. & $\begin{array}{l}\text { Applying a prediction algorithm based on detected mutual movement of markers and } \\
\text { fiducials, the planned robotic arm movement of the Cyberknife can be adjusted. } \\
\text { The main hard and software components are: } \\
\text { - } \quad \text { Camera array in the treatment room } \\
\text { " } \quad \text { Motion Tracking System (MTS) computer in equipment room } \\
\text { - Standard Cyberknife system with fiducial tracking facility. } \\
\text { " Synchrony identifies, updates and applies an adaptive correspondence model } \\
\text { between the internal target movements and external surface movements } \\
\text { throughout the entire treatment. } \\
\text { See Treatment Planning System }\end{array}$ \\
\hline \multirow{2}{*}{$\begin{array}{l}\text { Xsight }{ }^{\circledR} \\
\text { Xsight is a fiducial tracking software system, } \\
\text { attached to the Cyberknife system. }\end{array}$} & & \\
\hline & $\begin{array}{l}b . \\
c . \\
d .\end{array}$ & $\begin{array}{l}\text { Calculated position deviations are used for automatic couch reposition (during } \\
\text { patient set-up) or automatic robotic arm position correction during irradiation. } \\
\text { See Treatment Planning System. } \\
\text { Target related specific applications are available. } \\
\text { At the moment of writing this report: Xsight-Lung and Xsight-Spine. }\end{array}$ \\
\hline \multirow[b]{2}{*}{$\begin{array}{l}\text { Xsight-Lung® } \\
\text { Xsight-Lung is a module of the X-sight tracking } \\
\text { software system, attached to the Cyberknife system, } \\
\text { especially developed for lung target tracking. }\end{array}$} & & \\
\hline & a. & $\begin{array}{l}\text { Xsight-Lung enables tracking of moving solid lung targets based on a real-time } \\
\text { comparison of fiducials and DRR information. } \\
\text { It utilizes the differences in target and lung tissue density as landmark to track target } \\
\text { position. } \\
\text { Calculated position deviations are used for automatic couch reposition (during }\end{array}$ \\
\hline
\end{tabular}




\begin{tabular}{|c|c|}
\hline & $\begin{array}{l}\text { patient set-up) or automatic robotic arm position correction during irradiation. } \\
\text { d. See Synchrony. } \\
\text { e. See Treatment Planning System. }\end{array}$ \\
\hline \multicolumn{2}{|l|}{ Xsight-Spine® } \\
\hline $\begin{array}{l}\text { Xsight-Spine is a module of the X-sight tracking } \\
\text { software system, attached to the Cyberknife system, } \\
\text { especially developed for spine target tracking. }\end{array}$ & $\begin{array}{l}\text { a. Xsight-Spine enables tracking of spine targets based on a real-time comparison of a } \\
\text { grid with spine information at each grid-point as calculated during the treatment } \\
\text { planning process and the actual spine information per grid-point as determined with } \\
\text { X-rays during patient treatment. } \\
\text { b. Calculated position deviations are used for automatic couch reposition (during } \\
\text { patient set-up) or automatic robotic arm position correction during irradiation. } \\
\text { c. See Synchrony. } \\
\text { d. See Treatment Planning System. }\end{array}$ \\
\hline
\end{tabular}




\section{References}

[1] IAEA Human Health Reports, No. 7: Record and Verify Systems for Radiation Treatment of Cancer: Acceptance Testing, Commissioning and Quality Control. IAEA, https://www.iaea.org 2013.

[2] IAEA Safety Reports Series, No. 17: Lessons Learned from accidental exposures in Radiotherapy. IAEA, https://www.iaea.org 2000.

[3] ICRU Report 50: Prescribing, recording and reporting photon beam therapy. ICRU Report 50, http://www.icru.org 1993.

[4] ICRU Report 62: Prescribing, Recording and Reporting Photon Beam Therapy. ICRU Report 62, http://www.icru.org 1999.

[5] ICRU Report 83: Prescribing, Recording, and Reporting Photon-Beam Intensity-Modulated Radiation Therapy. ICRU Report 83, http://www.icru.org 2010.

[6] Implementation of the International Code of Practice on Dosimetry in Radiotherapy (TRS 398): Review of testing results. IAEA, https://www.iaea.org 2005.

[7] Radiation Protection $N^{\circ} 181$ : General guidelines on risk management in external beam radiotherapy. European Commission; ISSN 2315-2826, https://ec.europa.eu/energy/sites/ener/files/documents/RP181web.pdf 2015.

[8] Radiation Protection $\mathrm{N}^{\circ}$ 181: Technical supplement to Radiation Protection $\mathrm{n}^{\circ} 181$. European Commission, https://ec.europa.eu/energy/sites/ener/files/documents/RP181web.pdf 2015.

[9] Top 10 Health Technology Hazards for 2015. ECRI Institute, http://www.ecri.org 2014.

[10] Aalbers, A.H.L., et al., NCS Report 18: Code of Practice for the Absorbed Dose Determination in High Energy Photon and Electron Beams. Netherlands Commission on Radiation Dosimetry, http://radiationdosimetry.org 2012.

[11] ACCIRAD, European Commission, Patient safety in external beam radiotherapy - Guidelines on risk assessment and analysis of adverse events and near miss. Draft version April 2013. ACCIRAD, http://www.accirad.eu 2013.

[12] Alfonso, R., et al., A new formalism for reference dosimetry of small and nonstandard fields. Med. Phys. 35, 5179, 2008.

[13] Almond, P.R., et al. AAPM Report 51: protocol for clinical reference dosimetry of high-energy photon and electron beams. AAPM, http://www.aapm.org 1998. 
[14] Ammirati. M., et al., Stereotactic Radiotherapy of Central Nervous System and Head and Neck Lesions, Using a Conformal Intensity-Modulated Radiotherapy System (Peacocktrade mark System). Skull Base, 11,109, 2001.

[15] Anton, M., et al., Development of a secondary standard for the absorbed dose to water based on the alanine EPR dosimetry system. Appl. Radiat. Isot. 62, 779, 2005.

[16] Anton, M., et al., Is there an influence of the surrounding material on the response of the alanine dosimetry system? Phys. Med. Biol., 54, 2029, 2009.

[17] Araki, F., Monte Carlo study of a Cyberknife stereotactic radiosurgery system. Med. Phys., 33, 8, 2006.

[18] Araki, F., et al., Measurements of Gamma-Knife helmet output factors using a radiophotoluminescent glass rod dosimeter and a diode detector. Med. Phys.,30, 8, 2003.

[19] Arcovito, G., et al., Dose measurements and calculations of small radiation fields for 9-MV x rays. Med. Phys., 12, 779,1985.

[20] Arsenault, C., et al., Standards for Quality control at Canadian Radiation Treatment Centres: Stereotactic Radiosurgery/Radiotherapy. Canadian Association of Provincial Cancer Agencies (CAPCA), http://www.capca.ca 2005.

[21] Baldock, C., et al., Polymer gel dosimetry. Phys. Med. Biol., 55, R1, 2010.

[22] Benedict, S.H., et al., AAPM Report 101: Stereotactic body radiation therapy. AAPM, http://www.aapm.org 2010.

[23] Bjärngard, B.E., et al., Postal intercomparison of absorbed dose for high energy $\mathrm{x}$ rays with thermoluminescence dosimeters. Med. Phys., 7, 560, 1980.

[24] Blache, L., et al., IPEM Report 95: Risk Management and its Application to Medical Device Management. IPEM; ISBN 978-1903613-33-7, http://www.ipem.ac.uk 2007.

[25] Bond, J.E., et al., Comparison of an image registration technique based on normalized mutual information with a standard method. utilizing implanted markers in the staged radiosurgical treatment of large arteriovenous malformations. Int. J. Radiat. Oncol. Biol. Phys., 57, 1150, 2003.

[26] Bouchard, H., et al., A Monte Carlo method to evaluate the impact of positioning errors on detector response and quality correction factors in nonstandard beams. Phys. Med. Biol., 56, 2617, 2011. 
[27] Bruinvis, I.A.D., et al., NCS Report 15: Quality assurance of 3D-treatment planning systems for external photon and electron beams. Netherlands Commission on Radiation Dosimetry, http://radiationdosimetry.org 2005.

[28] Buis, D.R., et al., Delineation of brain AVM's on MR-angiography for the purpose of stereotactic radiosurgery. Int. J. Radiat. Oncol. Biol. Phys., 67, 308, 2007.

[29] Butson, M., et al., Radiochromic film for medical radiation dosimetry. Materials Science and Engineering, R 41, 2003.

[30] Capote, R., et al., An EGSnrc Monte Carlo study of the micro-ionization chamber for reference dosimetry of narrow irregular IMRT beamlets. Med. Phys., 31, 2416, 2004.

[31] Charles, P.H., et al., Monte Carlo-based diode design for correction-less small field dosimetry. Phys. Med. Biol., 58, $4501,2013$.

[32] Cheung, F.W., Law, M.Y., A novel conformity index for intensity modulated radiation therapy plan evaluation. Med. Phys., 39, 5740, 2012.

[33] Cosgrove, V.P., et al., Commissioning of a micro multi-leaf collimator and planning system for stereotactic radiosurgery. Rad. Oncol., 50, 325, 1999.

[34] Cranmer-Sargison, G., Monte Carlo modelling of diode detectors for small field MV photon dosimetry: detector model simplification and the sensitivity of correction factors to source parameterization. Phys. Med. Biol., 57, 5141, 2012.

[35] Cunningham, J., et al., Radiation Oncology Safety Information System (Rosis) - Profiles of Participants and their first 1074 Incident Reports. Rad. Oncol., 97, 601, 2010.

[36] Czarnecki, D., Zink, K., Monte Carlo calculated correction factors for diodes and ion chambers in small photon fields. Phys. Med. Biol., 58, 2431, 2013.

[37] Das, I.J., et al. , Choice of radiation detector in dosimetry of stereotactic radiosurgery-radiotherapy. J. Radiosurg., 3, $177,2000$.

[38] Das, I.J., et al. , Small fields: Nonequilibrium radiation dosimetry. Med. Phys., 35, 1, 2008.

[39] Das, S., et al., Accuracy of relocation, evaluation of geometric uncertainties and clinical target volume (CTV) to planning target volume (PTV) margin in fractionated stereotactic radiotherapy for intracranial tumors using relocatable Gill-Thomas-Cosman (GTC). J. Appl. Clin. Med. Phys., 12, 3260, 2010. 
[40] De Deene, Y., et al., The fundamental radiation properties of normoxic polymer gel dosimeters: a comparison between a methacrylic acid based and acrylamide based gels. Phys. Med. Biol., 51, 653, 2006.

[41] De Deene, Y., et al., Validation of MR-based polymer gel dosimetry as a preclinical three-dimensional verification tool in conformal radiotherapy. Magn. Reson. Med., 43, 116, 2000.

[42] De Vlamynck, K., et al., Dose measurements compared with Monte Carlo simulations of narrow 6 MV multileaf collimator shaped photon beams. Med. Phys., 26, 1874, 1999.

[43] Derreumaux, S., et al., A European quality assurance network for radiotherapy: dose measurement procedure. Phys. Med. Biol., 40, 1191, 1995.

[44] Devic, S., MRI simulation for radiotherapy treatment planning. Med. Phys., 39, 670, 2012.

[45] Dhabaan, A., et al., Six degrees of freedom CBCT-based positioning for intracranial targets treated with frameless stereotactic. J. Appl. Clin. Med. Phys., 13, 3916, 2012.

[46] Dieterich, S., Sherouse, G.W., Experimental comparison of seven commercial dosimetry diodes for measurement of stereotactic radiosurgery cone factors. Med. Phys., 7, 4166, 2011.

[47] Dieterich. S., et al., AAPM Report 135: Quality assurance for robotic radiosurgery. AAPM, http://www.aapm.org 2011.

[48] Ding, G.X., et al., Commissioning stereotactic radiosurgery beams using both experimental and theoretical methods. Phys. Med. Biol., 51, 2549, 2006.

[49] Ding, M., et al., Dosimetric comparison between 3DCRT and IMRT using different multileaf collimators in the treatment of brain tumors. Med. Dosim., 34, 1, 2009.

[50] Duggan, D. M. \& Coffey, C. W., Small photon field dosimetry for stereotactic radiosurgery. Med. Dosim., 23, $153,1998$.

[51] Editor: Podgorsak, E.B., Review of radiation oncology physics: A handbook for teachers and students. IAEA, Vienna, Austria, STI/PUB/1196 (ISBN 9201073046), 2005.

[52] Editor: Aspradakis, M.M. IPEM Report 103: Small Filed MV Photon Dosimetry. IPEM, ISBN 978-1-903613-45-0, http://www.ipem.ac.uk 2010. 
[53] Editor: Peyton, J.W.R., The learning cycle. Teaching and learning in medical practice. Rickmansworth, UK, Manticore, Europe Limited, 13-19, 1998.

[54] Editors: J. A. Purdy, J.A., Grant III, W., Palta, J.R, Butler, E.B., Perez, C.A., Image fusion for conformal radiation therapy. From: 3D Conformal Radiation Therapy and Intensity Modulated Radiation Therapy: Physics and Clinical Considerations, Advanced Medical Publishing, Madison, WI, USA., 2011.

[55] Editors: Bendict, S.H., Schlesingr, D.J., Goetsch, S.J., Kavanagh, B.D., Stereotactic Radiosurgery and Stereotactic Body Radiation Therapy. CRC Press, ISBN: 978-1-4398-4197-6, 2015.

[56] Editors: Kirby, M., Ryde, S., Hall, C., IPEM Report 94: Acceptance testing and commissioning of Linear Accelerators. IPEM, ISBN 978-1-903613-30-6, http://www.ipem.ac.uk 2006.

[57] Editors: M. Joiner, M., Kogel, van der, A., Basic Clinical Radiobiology. $4^{\text {th }}$ Edition, Hodder Arnold, ISBN 978-0-340-92966-7, 2009.

[58] Editors: Pawlicki, T., Dunscombe, P.B., Mundt, A.J., Scalliet, P., Imaging in Medical Diagnosis and Therapy: Quality and Safety in Radiotherapy. Taylor\&Francis, CRC Press; ISBN: 978-1-4398-0436-0, 2011.

[59] Eklund, K., Ahnesjo, A., Modelling silicon diode dose response factors for small photon fields. Phys. Med. Biol., 55, $7411,2010$.

[60] Ferreira, I.H., et al., The ESTRO-QUALity Assurance network (EQUAL) Rad. Oncol., 55, 2 73, 2000.

[61] Feuvret, L., et al., Conformity index : A Review. Int. J. Radiat. Oncol. Biol. Phys., 64, 333, 2006.

[62] Ford, E.C., et al., Evaluation of safety in a radiation oncology setting using failure mode and effect analysis. Int. J. Radiat. Oncol. Biol. Phys., 74, 852, 2009.

[63] Francescon, P., et al., Calculation of $k$ [sub Q[sub clin],Q[sub msr]][sup f[sub clin],f[sub msr]] for several small detectors and for two linear accelerators using Monte Carlo simulations. Med. Phys., 38, 6513, 2011.

[64] Francescon, P., et al., Total scatter factors of small beams: a multidetector and Monte Carlo study. Med. Phys., 35, $504,2008$.

[65] Francescon, P., et al., Use of a new type of radiochromic film, a new parallel-plate micro-chamber, MOSFETs, and TLD 800 microcubes in the dosimetry of small beams. Med. Phys., 25, 503, 1998.

[66] Fuss, M., et al., Repositioning accuracy of a commercially available thermoplastic mask system. Rad. Oncol., 71, $339,2004$. 
[67] Galerani, A. P., et al., Dosimetric impact of on-line correction via cone-beam CT-based image guidance for stereotactic lung radiotherapy. Int. J. Radiat. Oncol. Biol. Phys., 78, 1571, 2010.

[68] Guckenberger, M., et al., Reliability of the bony anatomy in image-guided stereotactic radiotherapy of brain metastases. Int. J. Radiat. Oncol. Biol. Phys., 69, 294, 2007.

[69] Habraken, M., et al., SAFER, Scenario analyse van faalwijzen, effecten en risico's. Uitgave ZonNW, 2006.

[70] Hendee, W.R., Herman, M.G., Improving patient safety in radiation oncology. Med. Phys., 38, 78, 2011.

[71] Herk, van, M., et al., Automatic registration of pelvic computed tomography data and magnetic resonance scans including a full circle method for quantitative accuracy evaluation. Med. Phys., 25, 2054, 1998.

[72] Heukelom, S., et al., Comparison of entrance and exit dose measurements using ionization chambers and silicon diodes. Phys. Med. Biol., 36, 47, 1991.

[73] Heydarian, M., et al., A comparison of dosimetry techniques in stereotactic radiosurgery. Phys. Med. Biol., 41, 93, 1996.

[74] Heydarian, M., et al., Dosimetric evaluation of a dedicated stereotactic linear accelerator using measurement and Monte Carlo simulation. Med. Phys., 35, 3943, 2008.

[75] Hoban, P.W., et al., Dose rate dependence of a PTW diamond detector in the dosimetry of a 6MV photon beam. Phys. Med. Biol., 39, 1219, 1994.

[76] Huq, M.S., et al., A Method for evaluation quality assurance needs in radiation therapy. Int. J. Radiat. Oncol. Biol. Phys., 71, S170, 2008.

[77] International Electrotechnical Commission International Standard, IEC 1217, Radiotherapy equipment, coordinates, movements and scales. International Electrotechnical Commission, Geneve, Suisse, 1996.

[78] Izewska J., Andreo P., The IAEAMHO TLD postal programme for radiotherapy hospitals. Rad. Oncol., 54, 65, 2000.

[79] Jackson, E.F., et al., AAPM Report 100: Acceptance Testing and Quality Assurance Procedures for Magnetic Resonance Imaging Facilities. AAPM, http://www.aapm.org 2010.

[80] Jeraj, R., et al., Dose calibration of nonconventional treatment systems applied to helical tomotherapy. Med. Phys., $32,2,2005$. 
[81] Kairn, T., et al., Clinical use of diodes and micro-chambers to obtain accurate small field output factor measurements. Australas. Phys. Eng. Sci. Med., 38, 357, 2015.

[82] Kataria, T., et al., Homogeneity Index: An objective tool for assessment of conformal radiation treatments. J. Med. Phys., 37, 207, 2012.

[83] Kerby, T., et al., Uncertainty analysis of absorbed dose calculation from thermoluminescence dosimeters. Med. Phys., 19, 1427, 1992.

[84] Klein, E.R., et al., AAPM Report 142: Quality assurance of medical accelerators. AAPM, http://www.aapm.org 2009.

[85] Knöös, T., et al., Volumetric and dosimetric evaluation of radiation treatment plans: radiation conformity index. Int. J. Radiat. Oncol. Biol. Phys., 42, 1169, 1998.

[86] Lake, F.R., et al., Teaching on the run tips. Medical Journal of Australia, $2004-2006$.

[87] Lárraga-Gutiérrez, J.M., et al., Properties of a commercial PTW-60019 synthetic diamond detector for the dosimetry of small radiotherapy beams. Phys. Med. Biol., 60, 905, 2015.

[88] Lasak, J.M., Gorecki, J.P., The history of stereotactic radiosurgery and radiotherapy. Otolaryngol. Clin. North. Am., 42, 593, 2009.

[89] Laub, W., Wong, T., The volume effect of detectors in the dosimetry of small fields used in IMRT. Med. Phys., 30, 341, 2003.

[90] Lee, H.R., et al., Evaluation and characterization of parallel plate microchamber's functionalities in small beam dosimetry. Med. Phys., 29, 2489, 2002.

[91] Leer, J.W.L., et al., A quality assurance system based on ISO standards: experience in a radiotherapy department. Rad. Oncol., 35, 75, 1995.

[92] Leksell, L., A stereotaxic apparatus for intracerebral surgery. Acta Chirurgica Scandinavica, 1949.

[93] Leksell, L., Stereotactic Radiosurgery. Journal of Neurology, Neurosurgery, and Psychiatry, 46, 797, 1983.

[94] Leksell, L., Jernberg, B., Stereotaxis and tomography. A technical note. Acta Neurochir. (Vienna), 52, 1, 1980.

[95] Lightstone, A.W., et al., AAPM Report 271: Intracranial stereotactic positioning systems. AAPM, http://www.aapm.org 2005.

[96] Lindquist, C., Paddick, I., The Leksell Gamma Knife Perfexion and comparisons with its predecessors. Neurosurgery, 61, 130, 2007. 
[97] Lindsay, P., et al., Investigation of energy dependency of EBT and EBT2 Gafchromic film. Med. Phys., 37, 2, 2010.

[98] Linthout, N., et al., Six dimensional analysis with daily stereoscopic x-ray imaging of intrafraction patient motion in head and neck treatments using five points fixation masks. Med. Phys., 33, 504, 2006.

[99] Liu, P.Z.Y., et al., Can small field diode correction factors be applied universally? Rad. Oncol., 112, 442, 2015.

[100] Lorenz, F., et al., An independent dose calculation algorithm for MLC-based stereotactic radiotherapy. Med. Phys., 34, $1605,2007$.

[101] Lydon, J.M., Theoretical and experimental validation of treatment planning for narrow MLC defined photon fields. Phys. Med. Biol., 50, 2701, 2005.

[102] Mack, A., et al., Quality Assurance in Stereotactic Radiosurgery / Radiotherapy according to DIN 6875-1. Stereotactic and Functional Neurosurgery, 82, 235, 2004.

[103] Mack, A., et al., Precision dosimetry for narrow photon beams used in radiosurgery-determination of Gamma Knife output factors. Med. Phys., 29, 2080, 2002.

[104] Maitz, A.H., et al., Quality assurance for gamma knife stereotactic radiosurgery. Int. J. Radiat. Oncol. Biol. Phys., 32, $1465,1995$.

[105] Marsolat, F., et al., A new single crystal diamond dosimeter for small beam: comparison with different commercial active detectors. Phys. Med. Biol., 58, 7647, 2013.

[106] Martens, C., et al., The value of pin-point ion chamber for characterization of small field segements used in intensity-modualted radiotherapy. Phys. Med. Biol., 45, 2519, 2000.

[107] McKenzie, A., et al., IPEM Report 92: Balancing Costs and Benefits of Checking in Radiotherapy. IPEM; ISBN 978-1-903613-27-6 http://www.ipem.ac.uk

[108] McKerracher, C., Thwaites, D,I., Assessment of new small-field detectors against standard-field detectors for practical stereotactic beam data acquisition. Phys. Med. Biol., 44, 2143, 1999.

[109] Meijer, G.J. (chairman), et al., NCS Report 9: Quality Control of Medical Linear Accelerators. Netherlands Commission on Radiation Dosimetry, http://radiationdosimetry.org 1996.

[110] Minniti, G., et al., Fractionated stereotactic radiotherapy for skull base tumors: analysis of treatment accuracy using a stereotactic mask fixation system. Radiat. Oncol., 5, 1, 2010. 
[111] Morales, J.E., et al., Dosimetry of cone-defined stereotactic radiosurgery fields with a commercial synthetic diamond detector. Med. Phys., 41, 111702, 2014.

[112] Nakamura, J.L., Dose conformity of gamma knife radiosurgery and risk factors for complications. Int. J. Radiat. Oncol. Biol. Phys., $51,1313,2001$.

[113] Naoi, Y., et al., A planning target volume margin formula for hypofractionated intracranial stereotactic radiotherapy under cone beam CT image guidance with a six-degrees-of-freedom robotic couch and a mouthpiece-assisted mask system: a preliminary study. Br. J. Radiol., 87, 1041, 2014.

[114] Nath, S. K., et al., Optically-guided frameless linac-based radiosurgery for brain metastases: clinical experience. J. Neurooncol., 97, 67, 2010.

[115] Niroomand-Rad, A., et al., AAPM Report 63: Radiochromic Film Dosimetry. AAPM, http://www.aapm.org 1998.

[116] Och, J.G., et al., AAPM Report 34: Acceptance Testing of Magnetic Resonance Imaging Systems. AAPM, http://www.aapm.org 1992.

[117] Oliver, M., et al., A treatment planning study comparing whole breast radiation therapy against conformal, IMRT and tomotherapy for accelerated partial breast irradiation. Rad. Oncol., 82, 317, 2007.

[118] Paddick, I., A simple scoring ratio to index the conformity of radiosurgical treatment plans. Technical note. J. Neurosurg. , Suppl. 3, $219,2000$.

[119] Paddick, I., Lippitz, B., A simple dose gradient measurement tool to complement the conformity index. J. Neurosurg., 105, 194, 2006.

[120] Pai, S., et al., AAPM Report 216: Radiographic film for megavoltage beam dosimetry. AAPM, http://www.aapm.org 2007.

[121] Palmans, H., et al., Ion recombination for ionization chamber dosimetry in a helical tomotherapy unit. Med. Phys., 37, $2876,2010$.

[122] Papas, E., et al., Small SRS photon field profile dosimetry performed using a PinPoint air ion chamber, a diamond detector, a novel silicon-diode array „DOSI..., and polymer gel dosimetry. Analysis and intercomparison. Med. Phys., 35, 10, 2008.

[123] Peng, L. C., et al., Quality assessment of frameless fractionated stereotactic radiotherapy using cone beam computed tomography. Int. J. Radiat. Oncol. Biol. Phys., 78, 1586, 2010. 
[124] Perks, J., et al., Dose quality assurance for stereotactic radiotherapy treatments. Phys. Med. Biol., 44, N209, 1999.

[125] Peters, A.R., et al., The accuracy of image registration for the brain and the nasopharynx using external anatomical landmarks. Phys. Med. Biol., 45, 2403, 2000.

[126] Pittomvils, G., et al., Measurement techniques, modelling strategies and pitfalls to avoid when implementing a mini-MLC in a nondedicated planning system. Strahl. Onkol., 183, 637, 2007.

[127] Podgorsak, E.B., Physics for radiosurgery with linear accelerators. Neurosurg. Clin. North. Am., 3, 9, 1992.

[128] Price, R.R., et al., AAPM Report 28: Quality Assurance methods and phantoms for Magnetic Resonance Imaging. AAPM, http://www.aapm.org 1990.

[129] Ralston, A., et al., Over-response of synthetic microDiamond detectors in small radiation fields. Phys. Med. Biol., 59, $5873,2014$.

[130] Ramaseshan, R., Heydarian, M., Comprehensive quality assurance for stereotatic radiosurgery treatments. Phys. Med. Biol., 48, N199, 2003.

[131] Rice, R.K., et al., Measurements of dose distributions in small beams of 6MV x-rays. Phys. Med. Biol., 32, $1087,1987$.

[132] Rowshanfarzad, P., et al., Isocenter verification for linac-based stereotactic radiation therapy: review of principles and techniques. J. Appl. Clin. Med. Phys., 12, 3645, 2011.

[133] Ruschin, M., et al., Performance of a novel repositioning head frame for gamma knife perfexion and image-guided linac-based intracranial stereotactic radiotherapy. Int. J. Radiat. Oncol. Biol. Phys., 78, 306, 2010.

[134] Sanchez-Doblado, F., et al., Ionisation chamber dosimetry of small photon fields: a Monte Carlo study on stopping-power ratios for radiosurgery and IMRT beams. Phys. Med. Biol., 48, 2081, 2003.

[135] Sanchez-Doblado. F., et al., A new method for output factor determination in MLC shaped narrow beams. Phys. Med. Biol., 23, $58,2007$.

[136] Schaeken, B., et al., Implementation of alanine/EPR as transfer dosimetry system in a radiotherapy audit program in Belgium. Rad. Oncol., 99, 1, 2011.

[137] Seuntjens, J., IAEA/AAPM Code of Practice for the Dosimetry of Static Small Photon Fields. Med. Phys., 42, 2015.

[138] Seuntjens, J., Standards for Non-Standard Photon Beams. Med. Phys., 42, 3739, 2015. 
[139] Seuntjes, J., Verhaegen, F., Comments on: Ionisation chamber dosimetry of small photon fields: a Monte Carlo study on stoppingpower ratios for radiosurgery and IMRT beams. Phys. Med. Biol., 48, L-43, 2003.

[140] Shaw, E., et al., Radiation Therapy Oncology Group: radiosurgery quality assurance guidelines. Int. J. Radiat. Oncol. Biol. Phys., 27, 1231, 1993.

[141] Shell, M.C., et al., AAPM Report 54: Stereotactic Radiosurgery. AAPM, http://www.aapm.org 1995.

[142] Solberg, T. D., et al., Quality assurance of immobilisation and target localization systems for frameless stereotactic cranial and extracranial hypofractionated radiotherapy. Int. J. Radiat. Oncol. Biol. Phys., (71-1 Suppl. ), S131, 2008.

[143] Solberg, T.D., et al., Quality and safety considerations in stereotactic radiosurgery and stereotactic body radiation therapy: Executive summary. Practical Radiation Oncology, 2, 2, 2012.

[144] Somigliana, A., et al., Dosimetry of Gamma Knife and linac-based radiosurgery using radiochromic and diode detectors. Phys. Med. Biol., 44, 887, 1999.

[145] Spadea, M. F., et al., Intra-fraction setup variability: IR optical localization vs. X-ray imaging in a hypofractionated patient population. Radiat. Oncol., 6, 38, 2011.

[146] Spang, F.J., et al., Photon small-field measurements with a CMOS active pixel sensor. Phys. Med. Biol., 60, $4383,2015$.

[147] Steenbeke, F., et al., Analysis of the targeting uncertainty of a stereotactic frameless radiosurgery technique for arteriovenous malformation. Rad. Oncol., 113, 371, 2014.

[148] Sturtewagen, E., et al., Multi-dimensional dosimetric verification of stereotactic radiotherapy for uveal melanoma using radiochromic EBT film. Zeitschrift für Medizinische Physik, 18, 27, 2008.

[149] Tagaste, B., et al., Comparison Between Infrared Optical and Stereoscopic X-ray Technologies for Patient Setup in Image Guided Stereotactic Radiotherapy. Int. J. Radiat. Oncol. Biol. Phys., 82, 1706, 2012.

[150] Taylor, M. L., et al., A contemporary review of stereotactic radiotherapy: inherent dosimetric complexities and the potential for detriment. Acta Oncol., 50, 483, 2011.

[151] Timothy, D., et al., Quality and safety considerations in stereotactic radiosurgery and stereotactic body radiation therapy: Executive summary. Practical Radiation Oncology, 2, 2, 2012. 
[152] Torres, A., et al., Testing the precision of stereotactic planning systems. Computerized Medical Imaging and Graphics, 22, 317, 1998.

[153] Tryggestad, E., et al., Inter- and Intrafraction Patient Positioning Uncertainties for Intracranial Radiotherapy: A Study of Four Frameless, Thermoplastic Mask-Based Immobilisation Strategies Using Daily Cone-Beam CT. Int. J. Radiat. Oncol. Biol. Phys., $80,281,2011$.

[154] Tyler, M., et al., Characterization of small-field stereotactic radiosurgery beams with modern detectors. Phys. Med. Biol., 58, 7595, 2013.

[155] Verellen, D., et al., Assessment of the Uncertainties in Dose Delivery of a Commercial System for Linac-based Stereotactic Radiosurgery. Int. J. Radiat. Oncol. Biol. Phys., 44, 2, 1999.

[156] Wagner, T.H., et al., A simple and reliable index for scoring rival stereotactic radiosurgery plans. Int. J. Radiat. Oncol. Biol. Phys., $57,1141,2003$.

[157] Wal van der, E. (chairman), et al., NCS Report 22: Code of Practice for the Quality Assurance and Control for Intensity Modulated Radiotherapy. Netherlands Commission on Radiation Dosimetry, http://radiationdosimetry.org 2013.

[158] Wang, J. Z., et al., Evaluation of patient setup uncertainty of optical guided frameless system for intracranial stereotactic radiosurgery. J. Appl. Clin. Med. Phys., 11, 3181, 2010.

[159] Westermark, M., et al., Comparative dosimetry in narrow high-energy photon beams. Phys. Med. Biol., 45, 685, 2000.

[160] Wiersma, R. D., et al., Development of a frameless stereotactic radiosurgery system based on real-time 6D position monitoring and adaptive head motion compensation. Phys. Med. Biol., 55, 389, 2010.

[161] Wu, A., et al., A dose homogeneity index for evaluating 192Ir interstitial breast implants. Med. Phys., 15, $104,1988$.

[162] Yomo, S., et al., A quantitative comparison of radiosurgical treatment parameters in vestibular schwannomas: the Leksell Gamma Knife Perfexion versus Model 4C. Acta Neurochir. (Vienna), 152, 47, 2010.

[163] Yoon, M., et al., A new homogeneity index based on statistical analysis of the dose-volume histogram. J. Appl. Clin. Med. Phys., 8, 9, 2007. 
[164] Younge, K.C., et al., Practical Implementation of Failure Mode and Effects Analysis for Safety and Efficiency in Stereotactic Radiosurgery. Int. J. Radiat. Oncol. Biol. Phys., 91, 1003, 2015.

[165] Zhang, B., et al., Development of a geometrically accurate imaging protocol at 3 Tesla MRI for stereotactic radiosurgery treatment planning. Phys. Med. Biol., 55, 6601, 2010. 


\section{Acknowledgement}

The members of the subcommittee would like to thank Jeroen van der Kamer (chairman NCS) and Frits Wittkämper for reading and reflecting on the manuscript. 